A preliminary version of this paper is published in the proceedings of the 16th International Conference on Applied Cryptography and Network Security-ACNS 2018. This is the full version.

\title{
Continuously Non-Malleable Codes with Split-State Refresh
}

\author{
Antonio Faonio ${ }^{1}$, Jesper Buus Nielsen ${ }^{2}$, Mark Simkin $^{2}$, and Daniele Venturi ${ }^{3}$ \\ ${ }^{1}$ IMDEA Software Institute, Madrid, Spain \\ ${ }^{2}$ Aarhus University, Aarhus, Denmark \\ ${ }^{3}$ Sapienza University of Rome, Rome, Italy
}

June 15, 2018

\begin{abstract}
Non-malleable codes for the split-state model allow to encode a message into two parts, such that arbitrary independent tampering on each part, and subsequent decoding of the corresponding modified codeword, yields either the same as the original message, or a completely unrelated value. Continuously non-malleable codes further allow to tolerate an unbounded (polynomial) number of tampering attempts, until a decoding error happens. The drawback is that, after an error happens, the system must self-destruct and stop working, otherwise generic attacks become possible.

In this paper we propose a solution to this limitation, by leveraging a split-state refreshing procedure. Namely, whenever a decoding error happens, the two parts of an encoding can be locally refreshed (i.e., without any interaction), which allows to avoid the self-destruct mechanism in some applications. Additionally, the refreshing procedure can be exploited in order to obtain security against continual leakage attacks. We give an abstract framework for building refreshable continuously non-malleable codes in the common reference string model, and provide a concrete instantiation based on the external Diffie-Hellman assumption.

Finally, we explore applications in which our notion turns out to be essential. The first application is a signature scheme tolerating an arbitrary polynomial number of split-state tampering attempts, without requiring a self-destruct capability, and in a model where refreshing of the memory happens only after an invalid output is produced. This circumvents an impossibility result from a recent work by Fuijisaki and Xagawa (Asiacrypt 2016). The second application is a compiler for tamper-resilient read-only RAM programs. In comparison to other tamper-resilient RAM compilers, ours has several advantages, among which the fact that, in some cases, it does not rely on the self-destruct feature.
\end{abstract}

Keywords: non-malleable codes; tamper-resilient cryptography; split-state model. 


\section{Contents}

1 Introduction 1

1.1 Our Contributions . . . . . . . 2

1.2 Code Construction ....... . 3

1.3 Limitations of the Refresh Paradigm ......... 4

1.4 Further Related Work ..... . 5

2 Preliminaries and Building Blocks 5

2.1 Basic Notation . . . . . . . . 5

2.2 Non-Interactive Zero-Knowledge 6

2.3 Public-Key Encryption . . . . . . 8

2.4 Commitment Schemes . . . . . . 9

2.5 Signature Schemes . . . . . . . 10

2.6 Authenticated Encryption . . . . 11

3 Non-Malleability with Refresh 12

3.1 Split-State Codes . . . . . . . . 12
3.2 The Definition . . . . . . . . 12

4 Code Construction $\quad 14$

4.1 Proof Intuition . . . . . . . 15

4.2 Description of the Simulator . . . 17

4.3 Hybrids . . . . . . . . . . . 19

4.4 Indistinguishability of the Hybrids 21

5 Concrete Instantiations $\quad 30$

5.1 The PKE Scheme . . . . . . . . 31

5.2 The Commitment Scheme . . . . 32

5.3 The Label-Malleable NIZK . . . 32

6 Applications $\quad 34$

6.1 Tamper-Resilient Signatures . . . 34

6.2 Tamper-Resilient RAM $\ldots . . .37$

$\begin{array}{lll}7 & \text { Conclusion } & 47\end{array}$

\section{Introduction}

Tampering attacks are subtle attacks that undermine the security of cryptographic implementations by exploiting physical phenomena that allow to modify the underlying secrets. Indeed, a long line of works (see, e.g., $[12,10,42,53])$ has established that black-box interaction with a tampered implementation can potentially expose the entire content of the secret memory. Given this state of affairs, protecting cryptographic schemes against tampering attacks has become an important goal for modern cryptographers.

An elegant solution to the threat of tampering attacks against the memory comes from the notion of non-malleable codes (NMCs), put forward by Dziembowski, Pietrzak, and Wichs [32]. Intuitively, a non-malleable encoding (Encode, Decode) allows to encode a value $M$ into a codeword $C \leftarrow \operatorname{Encode}(M)$, with the guarantee that a modified codeword $\widetilde{C}=f(C)$ w.r.t. a tampering function $f \in \mathcal{F}$, when decoded, yields either $M$ itself, or a completely unrelated value. An important parameter for characterizing the security guarantee offered by NMCs is the class of modifications $\mathcal{F}$ that are supported by the scheme. Since non-malleability is impossible to obtain for arbitrary (albeit efficient) modifications, ${ }^{1}$ research on NMCs has focused on constructing such schemes in somewhat restricted, yet interesting, models. One such model that has been the focus of intensive research (see, e.g., $[50,36,3,2]$ ) is the split-state model, where the codeword $C$ consists of two parts $\left(C_{0}, C_{1}\right)$ that can be modified independently (yet arbitrarily). This setting is also the focus of this paper.

Unfortunately, standard NMCs protect only against a single tampering attack. ${ }^{2}$ To overcome this limitation, Faust et al. [36] introduced continuously non-malleable codes (CNMCs for short), where the attacker can tamper for an unbounded (polynomial) number of times with

\footnotetext{
${ }^{1}$ As it can be seen by considering the tampering function that first decodes the codeword, flips one bit of the message, and then encodes the result.

${ }^{2}$ When using NMCs to obtain security against memory tampering, one can still obtain security against continuous attacks by enforcing a re-encoding of the secret key after each invocation; however, this comes with several disadvantages [33], among which the fact that the encoding process is considerably more complex than the decoding process.
} 
the codeword, until a decoding error happens which triggers the self-destruction of the device. As argued in [36], the self-destruct capability is necessary, as each decoding error might be used to signal one bit of information about the target codeword.

Another desirable feature of non-malleable codes is their ability to additionally tolerate leakage attacks, by which the adversary can obtain partial information on the codeword while performing a tampering attack. Note that in the split-state model this means that the adversary can leak independently from the two parts $C_{0}$ and $C_{1}$. All previous constructions of leakageresilient NMCs either achieve security in the so-called bounded-leakage model [50, 36, 4], where the total amount of leakage (from each part) is upper-bounded by a value $\ell$ that is a parameter of the scheme, or only satisfy non-continuous non-malleability [33].

\subsection{Our Contributions}

We introduce a new form of CNMCs (dubbed R-CNMCs) that include a split-state algorithm for refreshing a valid codeword. The refresh procedure is invoked either after a decoding error happens, or in order to amplify resilience to leakage, and takes place directly on the memory and without the need of a central unit. Our new model has two main attractive features, which we emphasize below.

- It captures security in the so-called continual noisy-leakage model, where between each refresh the adversary can leak an arbitrary (yet independent) amount of information on the two parts $C_{0}, C_{1}$, as long as the leakage does not reveal (information-theoretically) more than $\ell$ bits of information. Importantly, this restriction is well-known to better capture realistic leakage attacks.

- It avoids the need for the self-destruct capability in some applications. Besides mitigating simple denial-of-service attacks, this feature is useful in situations where a device (storing an encoding of the secret state) is not in the hands of the adversary (e.g., because it has been infected by a malware), as it still allows to (non-interactively) refresh the secret state and continue to safely use the device in the wild.

Our first contribution is an abstract framework for constructing R-CNMCs, which we are able to instantiate under the external Diffie-Hellman assumption. This constitutes the first NMC that achieves at the same time continuous non-malleability and security under continual noisy leakage, in the split-state model (assuming an untamperable common reference string).

Next, we explore applications of R-CNMCs. As second contribution, we show how to construct a split-state ${ }^{3}$ signature scheme resilient to continuous tampering and leakage attacks, without relying on the self-destruct capability, and where the memory content is refreshed in case a decoding error is triggered. Interestingly, Fujisaki and Xagawa [40] recently showed that such a notion is impossible to achieve for standard (i.e., non split-state) signature schemes, even if the self-destruct capability is available; hence, our approach can be interpreted as a possible way to circumvent the impossibility result in [40].

Our third contribution consists of two generic compilers for protecting random access machine (RAM) computations against tampering attacks [24, 37]. Here, we build on the important work of Dachman-Soled et al. [24], who showed how to compile any RAM to be resilient to continual tampering and leakage attacks, by relying both on an update and a self-destruct mechanism. We refer the reader to Section 6.2 for further details on our RAM compilers. Below, we highlight the main technical ideas behind our code construction.

\footnotetext{
${ }^{3}$ This means that the signing key is made of two shares that are stored in two separate parts of the memory, and need to be combined upon signing.
} 


\subsection{Code Construction}

The starting point of our code construction is the recent work of Faonio and Nielsen [33]. The scheme built in [33] follows a template that originates in the work of Liu and Lysyanskaya [50], in which the left side of the encoding stores the secret key $s k$ of a public-key encryption (PKE) scheme, whereas the right side of the encoding stores a ciphertext $c$, encrypting the encoded message $M$, plus a non-interactive zero-knowledge (NIZK) argument that proves knowledge of the secret key under the label $c$; the PKE scheme is chosen to be a continual-leakage resilient storage friendly PKE (CLRS friendly PKE for short) scheme (see Dodis et al. [29]), whereas the NIZK is chosen to be a malleable NIZK argument of knowledge (see Chase et al. [15]). Such a code was shown to admit a split-state refresh procedure, and, at the same time, to achieve bounded-time non-malleability.

The NM code of [33] does not satisfy security against continuous attacks. In fact, an attacker can create two valid codewords $\left(C_{0}, C_{1}\right)$ and $\left(C_{0}, C_{1}^{\prime}\right)$ such that $\operatorname{Decode}\left(C_{0}, C_{1}\right) \neq$ Decode $\left(C_{0}, C_{1}^{\prime}\right)$. Given this, the adversary can tamper the left side to $C_{0}$ and the right side to either $C_{1}$ or $C_{1}^{\prime}$ according to the bits of the right side of the target encoding. This way, assuming tampering is non-persistent, the adversary can leak all the bits of $C_{1}$ without activating the self-destruct mechanism. More in general, for any CNMC it should be hard to find two valid codewords $\left(C_{0}, C_{1}\right)$ and $\left(C_{0}, C_{1}^{\prime}\right)$ such that Decode $\left(C_{0}, C_{1}\right) \neq \operatorname{Decode}\left(C_{0}, C_{1}^{\prime}\right)$. This property, called "message uniqueness", was originally defined in [36]. ${ }^{4}$

Going back to the described code construction, an attacker can sample a secret key sk and create two ciphertexts, $c_{0}$ for $M$ and $c^{\prime}$ for $M^{\prime}$, where $M \neq M^{\prime}$, together with the corresponding honestly computed NIZKs, and thus break message uniqueness. We fix this issue by further binding the right and the left side of an encoding. To do so, while still being able to refresh the two parts independently, we keep untouched the structure of the right side of the codeword, but we change the message that it carries. Specifically, the ciphertext $c$ in our code encrypts the message $M$ concatenated with the randomness $r$ for a commitment $\gamma$ that is stored in the left side of the codeword together with the secret key for the PKE scheme. Observe that "message uniqueness" is now guaranteed by the binding property of the commitment scheme. Our construction additionally includes another NIZK for proving knowledge of the committed value under the label $s k$, in order to further link together the left and the right side of the codeword.

Proof strategy. Although our construction shares similarities with previous work, our proof techniques diverge significantly from the ones in [33,36]. The main trick of [36] is to show that given one half of the codeword it is possible to fully simulate the view of the adversary in the tampering experiment, until a decoding error happens. To catch when a decoding error happens, [36] carries on two independent simulators in an interleaved fashion; as they prove, a decoding error happens exactly when the outputs of the two simulations diverge. The main obstacle they faced is how to succinctly compute the index where the two simulations diverge, so that non-malleability can be reduced to the security of the inner leakage-resilient storage scheme (see Daví et al. [27]) they rely on. To solve this, [36] employs an elegant dichotomous search-by-hash strategy over the partial views produced by the two simulators. At this point the experiment can terminate, and thanks to a specific property of the leakage-resilient storage scheme, the simulator can "extract" the decoded value corresponding to a tampered codeword.

\footnotetext{
${ }^{4}$ Faust et al. also consider "codeword uniqueness", where the fact that $\operatorname{Decode}\left(C_{0}, C_{1}\right) \neq \operatorname{Decode}\left(C_{0}, C_{1}^{\prime}\right)$ is not required (as long as $C_{1} \neq C_{1}^{\prime}$ ). However, this flavor of uniqueness only allows to rule out so-called continuous super non-malleability, where one asks that not only the decoded value, but the entire modified codeword, be independent of the message. It is easy to see that no R-CNMC can satisfy "codeword uniqueness", as for instance $C_{1}^{\prime}$ could be obtained as a valid refresh of $C_{1}$.
} 
Unfortunately, we cannot generalize the above proof strategy to multiple rounds. Indeed, Faust et al. exploit the fact that the leakage-resilient storage scheme remains secure even when the adversary is allowed to obtain one half of the encoding in full. Clearly, after that, the adversary is not allowed to leak further from the other half of the codeword. In our case, we would need to repeat the above trick again and again, in particular after each decoding error (and subsequent refresh of the target encoding) happens; however, once the reduction obtains one half of the codeword it cannot ask leakage queries anymore, so that it is unclear how to complete the proof. We give a solution to this problem by relying on a simple informationtheoretic observation.

Let $\left(X_{0}, X_{1}\right)$ be two random variables, and consider a process that interleaves the computation of a sequence of leakage functions $g^{1}, g^{2}, g^{3}, \ldots$ from $X_{0}$ and from $X_{1}$. The process continues until, for some index $i \in \mathbb{N}$, we have that $g^{i}\left(X_{0}\right) \neq g^{i}\left(X_{1}\right)$. We claim that $\bar{g}^{i}\left(X_{0}\right):=g^{1}\left(X_{0}\right), g^{2}\left(X_{0}\right), \cdots, g^{i-1}\left(X_{0}\right)$ do not reveal more information about $X_{0}$ than what $X_{1}$ and the index $i$ already reveal. To see this, consider $\widetilde{\mathbb{H}}_{\infty}\left(X_{0} \mid \bar{g}^{i}\left(X_{0}\right)\right)$, i.e. the average conditional min-entropy of $X_{0}$, which is, roughly speaking, the amount (on average) of uncertainty of $X_{0}$ given $\bar{g}^{i}\left(X_{0}\right)$ as side information. (See Section 2.1 for the formal definition.) Now, since $\bar{g}^{i}\left(X_{0}\right)$ and $\bar{g}^{i}\left(X_{1}\right)$ are exactly the same random variables we can derive ${ }^{5}$ :

$$
\widetilde{\mathbb{H}}_{\infty}\left(X_{0} \mid \bar{g}^{i}\left(X_{0}\right)\right)=\widetilde{\mathbb{H}}_{\infty}\left(X_{0} \mid \bar{g}^{i}\left(X_{1}\right)\right) \geq \widetilde{\mathbb{H}}_{\infty}\left(X_{0} \mid X_{1}, i\right) .
$$

The latter implies that the size of the view of the adversary, although potentially much larger than the leakage bound, might reveal only little information.

One can use the above observation in order to give an alternative proof of security for the scheme in [36], where the reduction to the leakage-resilient storage scheme loses only a factor $O(\kappa)$ in the leakage bound (instead of $O(\kappa \log \kappa)$ ). Briefly, the idea is to carry on two independent simulators in an interleaved fashion (as in [36]) and, at each invocation, outputting first the hashes ${ }^{6}$ of the simulated tampered codeword, and then, if the hashes match, leak the full simulated tampered codeword. This allows to avoid the dichotomous search-by-hash strategy, and thus to limit the total amount of leakage to $O(\kappa)$.

\subsection{Limitations of the Refresh Paradigm}

The original motivation behind the concept of CNMCs, as discussed in [36], was to make the standard tampering application of NMCs more realistic, in the following sense. Imagine that the device we want to protect, besides storing an encoding of the secret key, also contains other non-encoded data. For example the device could be a laptop, and tampering attacks could be launched by a virus with read and write access to the memory. The virus could exploit the space available on the device to make multiple copies of the original codeword, which thus can be tampered continuously. However, since the underlying NMC is resilient to continuous attacks, the privacy of the secret key is still preserved. Intuitively, this works because the adversary, to gather information about the codeword, needs to trigger a decoding error, however, once such an event happens the device self-destructs and the game is over.

While our notion of R-CNMCs allows for the very same application as considered in [36] (as any R-CNMC is in particular a CNMC), the straight-forward extension of the above scenario to a setting where the self-destruct is not available, but the encoding of the key is refreshed after a decoding error happens, does not work. To make the attack even harder, suppose that all the locations in the memory are encoded using a R-CNMC, and whenever a decoding error happens,

\footnotetext{
${ }^{5}$ In the last equation, we also use that the output of a function is at most as informative as the input.

${ }^{6} \mathrm{By}$ collision resistance of the hash function, if the two hashes match then the simulated tampered codewords are the same for both the simulators.
} 
the full memory is refreshed. An adversary could perform the following tampering attack. In case the $i$-th bit of the attacked codeword is equal to 1 , the $i$-th location of the memory is corrupted (i.e., it is replaced with an invalid codeword), otherwise it is left untouched. Thus, the adversary reads sequentially all the memory locations. Very soon a decoding error will be triggered and all the codewords refreshed, however the information of the original codeword is still present in the memory (as the untouched codewords will be refreshed to valid codewords, while the corrupted codewords will still trigger a decoding error). This means that eventually the adversary is able to fully retrieve the original codeword (even if it has been refreshed many times), which leads to a clear breach of non-malleability.

\section{$1.4 \quad$ Further Related Work}

Several constructions of non-malleable codes in the split-state model appeared in the literature, both for the information-theoretic [32, 31, 3, 20, 4, 2, 14, 5, 48, 49, 17] and computational setting [50, 36, 24, 1, 46, 52, 23]. Non-malleable codes exist also for several other models besides split-state tampering, including bit-wise independent tampering and permutations [20, $6,7,22,21]$, circuits of polynomial size [32, 19, 38, 39], constant-state tampering [18], block-wise tampering [13], space-bounded algorithms [35, 9], bounded-depth circuits [8, 16], and partial functions [47].

The concept of non-malleable codes with split-state refresh was recently proposed in [33]. The main difference with our work is that in [33] the refresh mechanism essentially allows to only tolerate continual leakage, but the codeword needs to be refreshed at each invocation in order to achieve security against continuous tampering attacks. In contrast, R-CNMCs satisfy both resilience to continual leakage and to continuous tampering without invoking a refresh at each invocation.

Security against tampering attacks against the memory can also be obtained without relying on non-malleable codes. See, e.g., [45, 25, 40, 34, 26] (and the references therein) for some examples.

\section{Preliminaries and Building Blocks}

\subsection{Basic Notation}

We let $\mathbb{N}$ denote the naturals and $\mathbb{R}$ denote the reals. For $a, b \in \mathbb{R}$, we define $[a, b]=\{x \in$ $\mathbb{R}: a \leq x \leq b\} ;$ for $a \in \mathbb{N}$ we let $[a]=\{0,1, \ldots, a\}$. If $x$ is a bit-string, we denote its length by $|x|$, and, for any $i \leq|x|$, we let $x[i]$ be the $i$-th bit of $x$. If $\mathcal{X}$ is a set, $|\mathcal{X}|$ represents the number of elements in $\mathcal{X}$. When $x$ is chosen randomly in $\mathcal{X}$, we write $x \leftarrow \mathcal{X}$. When $\mathrm{A}$ is an algorithm, we write $y \leftarrow \mathrm{A}(x)$ to denote a run of $\mathrm{A}$ on input $x$ and output $y$; if $\mathrm{A}$ is randomized, then $y$ is a random variable and $\mathrm{A}(x ; r)$ denotes a run of $\mathrm{A}$ on input $x$ and random coins $r$. An algorithm $\mathrm{A}$ is probabilistic polynomial-time (PPT) if $\mathrm{A}$ is allowed to make random choices, and the computation of $\mathrm{A}(x ; r)$ terminates in at most a polynomial number of steps (in $|x|)$, for any input $x \in\{0,1\}^{*}$ and randomness $r \in\{0,1\}^{*}$.

Indistinguishability and min-entropy. We let $\kappa \in \mathbb{N}$ be a security parameter. A function $\nu: \mathbb{N} \rightarrow[0,1]$ is called negligible in $\kappa$ (or simply negligible) if it vanishes faster than the inverse of any polynomial in $\kappa$. For two ensembles $\mathcal{X}=\left\{X_{\kappa}\right\}_{\kappa \in \mathbb{N}}$ and $\mathcal{Y}=\left\{Y_{\kappa}\right\}_{\kappa \in \mathbb{N}}$, we write $\mathcal{X} \approx_{c} \mathcal{Y}$, meaning that every PPT distinguisher $\mathrm{D}$ has negligible advantage in distinguishing $\mathcal{X}$ and $\mathcal{Y}$, i.e., $\left|\mathbb{P}\left[\mathrm{D}\left(X_{\kappa}\right)=1\right]-\mathbb{P}\left[\mathrm{D}\left(Y_{\kappa}\right)=1\right]\right| \leq \nu(\kappa)$ where $\nu(\kappa)$ is a negligible function. Similarly, we write $\mathcal{X} \approx_{s} \mathcal{Y}$ for statistical indistinguishability between $\mathcal{X}$ and $\mathcal{Y}$, meaning that 
the above indistinguishability holds for all (even computationally unbounded) distinguishers (or, equivalently, that the statistical distance between $\mathcal{X}$ and $\mathcal{Y}$ is negligible).

The min-entropy of a random variable $X$, defined over a set $\mathcal{X}$, is denoted as $\mathbb{H}_{\infty}(X):=$ $-\log \max _{x \in \mathcal{X}} \mathbb{P}[X=x]$ and represents the best chance of guessing $X$ by an unbounded adversary. Conditional average min-entropy captures how hard it is to guess $X$ on average, given some side information $Z \in \mathcal{Z}$ (possibly related to $X$ ), and it is denoted as $\widetilde{\mathbb{H}}_{\infty}(X \mid Z):=$ $-\log \mathbb{E}_{z \in \mathcal{Z}} \max _{x \in \mathcal{X}} \mathbb{P}[X=x \mid Z=z]$. We rely on the following lemmata [30].

Lemma 1. Let $X$ and $Z$ be possibly correlated random variables, and let $f$ be any (possibly randomized) function. Then, $\widetilde{\mathbb{H}}_{\infty}(X \mid f(Z)) \geq \widetilde{\mathbb{H}}_{\infty}(X \mid Z)$.

Lemma 2. Let $X, Z_{1}, Z_{2}$ be possibly correlated random variables, where $Z_{2}$ takes at most $2^{\ell}$ values. Then, $\widetilde{\mathbb{H}}_{\infty}\left(X \mid Z_{1}, Z_{2}\right) \geq \widetilde{\mathbb{H}}_{\infty}\left(X \mid Z_{1}\right)-\ell$.

Following [28], we define a notion of a function being $\ell$-leaky.

Definition 1 ( $\ell$-leaky function). A (possibly randomized) function $g:\{0,1\}^{*} \rightarrow\{0,1\}^{*}$ is $\ell$-leaky, if for all $X$ distributed over $\{0,1\}^{*}$ we have that $\widetilde{\mathbb{H}}_{\infty}(X \mid g(X)) \geq \mathbb{H}_{\infty}(X)-\ell$.

As shown in [28, Lemma L.3] the above notion composes nicely, meaning that if two functions are respectively $\ell_{1}$-leaky and $\ell_{2}$-leaky, their concatenation is $\left(\ell_{1}+\ell_{2}\right)$-leaky.

Oracle machines. Given a pair of strings $X=\left(X_{0}, X_{1}\right) \in\left(\{0,1\}^{*}\right)^{2}$ define the oracle $\mathcal{O}_{\infty}(X)$ to be the split-state leakage oracle that takes as input tuples of the form $(\beta, g)$, where $\beta \in\{0,1\}$ is an index and $g$ is a function described as a circuit, and outputs $g\left(X_{\beta}\right)$. An adversary A with oracle access to $\mathcal{O}_{\infty}(X)$ is called $\ell$-valid, for some $\ell \in \mathbb{N}$, if for all $\beta \in\{0,1\}$ the concatenation of the leakage functions sent by $\mathrm{A}$ is an $\ell$-leaky function of $X_{\beta}$.

Given two PPT interactive algorithms $\mathrm{A}$ and $\mathrm{B}$ we write $\left(y_{\mathrm{A}} ; y_{\mathrm{B}}\right) \leftarrow\left(\mathrm{A}\left(x_{\mathrm{A}}\right) \leftrightarrows \mathrm{B}\left(x_{\mathrm{B}}\right)\right)$ to denote the execution of algorithm $\mathrm{A}$ (with input $x_{\mathrm{A}}$ ) and algorithm $\mathrm{B}$ (with input $x_{\mathrm{B}}$ ). The string $y_{\mathrm{A}}$ (resp. $y_{\mathrm{B}}$ ) is the output of $\mathrm{A}$ (resp. B) at the end of such interaction. In particular, we write $\mathrm{A} \leftrightarrows \mathcal{O}_{\infty}(X)$ to denote $\mathrm{A}$ having oracle access to the leakage oracle with input $X$. Moreover, we write $\mathrm{A} \leftrightarrows \mathrm{B}, \mathrm{C}$ to denote $\mathrm{A}$ interacting in an interleaved fashion both with $\mathrm{B}$ and with C.

\subsection{Non-Interactive Zero-Knowledge}

Let $\mathcal{R} \subseteq\{0,1\}^{*} \times\{0,1\}^{*}$ be an NP-relation; the language associated with $\mathcal{R}$ is $\mathcal{L}_{\mathcal{R}}:=\{x$ : $\exists w$ s.t. $(x, w) \in \mathcal{R}\}$. We typically assume that given a pair $(x, w)$ it is possible to efficiently verify whether $(x, w) \in \mathcal{R}$ or not. Roughly, a non-interactive argument (NIA) for an NP-relation $\mathcal{R}$ allows to create non-interactive proofs for statements $x \in \mathcal{L}$, when additionally given a valid witness $w$ corresponding to $x$. More formally, a NIA $\mathcal{N} \mathcal{I} \mathcal{A}:=($ CRSGen, Prove, Ver) for $\mathcal{R}$, with label space $\Lambda$, is a tuple of PPT algorithms specified as follows: (1) The (randomized) initialization algorithm CRSGen takes as input the security parameter $1^{\kappa}$, and creates a common reference string (CRS) $\omega \in\{0,1\}^{*}$; (2) The (randomized) prover algorithm Prove takes as input the CRS $\omega$, a label $\lambda \in \Lambda$, and a pair $(x, w)$ such that $(x, w) \in \mathcal{R}$, and produces a proof $\pi \leftarrow \operatorname{Prove}^{\lambda}(\omega, x, w) ;(3)$ The (deterministic) verifier algorithm Ver takes as input the CRS $\omega$, a label $\lambda \in \Lambda$, and a pair $(x, \pi)$, and outputs a decision $\operatorname{bit} \operatorname{Ver}^{\lambda}(\omega, x, \pi)$.

Completeness means that for all CRSs $\omega$ output by CRSGen $\left(1^{\kappa}\right)$, for all labels $\lambda \in \Lambda$, and for all pairs $(x, w) \in \mathcal{R}$, we have that $\operatorname{Ver}^{\lambda}\left(\omega, x, \operatorname{Prove}^{\lambda}(\omega, x, w)\right)=1$ with all but a negligible probability. Below, we define the security properties we require for NIAs. The first property, known as adaptive multi-theorem zero-knowledge, intuitively says that honestly computed proofs do not reveal anything beyond the validity of the statement. 


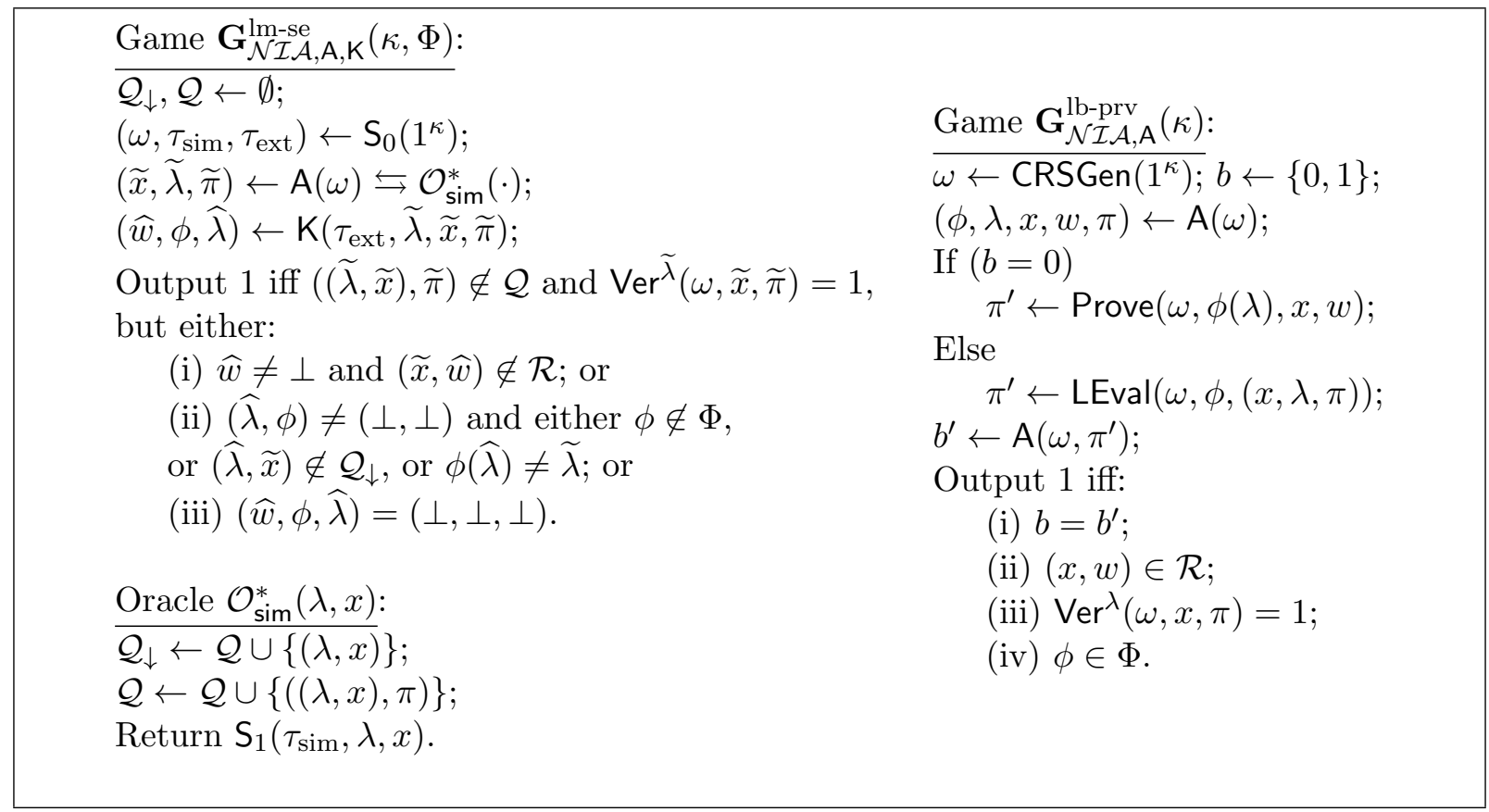

Figure 1: Games defining $\Phi$-malleable label simulation extractability, and label simulation privacy, of a NIA $\mathcal{N} \mathcal{I} \mathcal{A}$.

Definition 2 (Adaptive multi-theorem zero-knowledge). Let $\mathcal{N} \mathcal{I} \mathcal{A}$ be a NIA for a relation $\mathcal{R}$. We say that $\mathcal{N} \mathcal{I} \mathcal{A}$ satisfies adaptive multi-theorem zero-knowledge if the following holds:

(i) There exists a PPT algorithm $\mathrm{S}_{0}$ that outputs a CRS $\omega$, and a simulation trapdoor $\tau_{\text {sim. }}$.

(ii) There exist a PPT simulator $\mathrm{S}_{1}$ and a negligible function $\nu: \mathbb{N} \rightarrow[0,1]$ such that, for all PPT adversaries $A$, we have

$$
\begin{aligned}
\mid \mathbb{P}[\mathrm{A}(\omega) \leftrightarrows & \left.\mathcal{O}_{\mathrm{prv}}(\omega, \cdot)=1: \omega \leftarrow \operatorname{CRSGen}\left(1^{\kappa}\right)\right] \\
& -\mathbb{P}\left[\mathrm{A}(\omega) \leftrightarrows \mathcal{O}_{\text {sim }}\left(\tau_{\text {sim }}, \cdot\right)=1:\left(\omega, \tau_{\text {sim }}\right) \leftarrow \mathrm{S}_{0}\left(1^{\kappa}\right)\right] \mid \leq \nu(\kappa)
\end{aligned}
$$

In the above equation, both oracles $\mathcal{O}_{\text {prv }}$ and $\mathcal{O}_{\text {sim }}$ take as input a tuple $(\lambda, x, w)$ and check if $(x, w) \in \mathcal{R}$ : If this is the case, $\mathcal{O}_{\text {prv }}$ returns $\operatorname{Prove}^{\lambda}(\omega, x, w)$, whereas $\mathcal{O}_{\text {sim }}$ ignores $w$ and outputs a simulated argument $\mathrm{S}_{1}\left(\tau_{\text {sim }}, \lambda, x\right)$; otherwise, both oracles return $\perp$.

Roughly speaking, a NIA satisfies soundness if no efficient malicious adversary can create an accepting proof for a false statement (i.e., for a statement $x$ such that $x \notin \mathcal{L}$ ). Knowledge soundness is a stronger form of soundness, essentially demanding that we can efficiently extract a witness from any accepting proof created by an efficient adversary. Our construction will be based on a so-called label-malleable NIA, parameterized by a set of label transformations $\Phi$, where for any $\phi \in \Phi$, the co-domain of $\phi$ is a subset of $\Lambda$. For such NIAs, given a proof $\pi$ under some label $\lambda \in \Lambda$, and the CRS $\omega$, one can efficiently generate new proofs $\pi^{\prime}$ for the same statement under a different label $\phi(\lambda)$, for any $\phi \in \Phi$ (without knowing a witness); this is formalized via an additional (randomized) label-derivation algorithm LEval, which takes as input the CRS $\omega$, a transformation $\phi \in \Phi$, a label $\lambda \in \Lambda$, and a pair $(x, \pi)$, and outputs a new proof $\pi^{\prime}$. The property below intuitively says that a NIA satisfies knowledge soundness, except that labels are malleable w.r.t. $\Phi$. 
Definition 3 ( $\Phi$-Malleable label simulation extractability). Let $\mathcal{N} \mathcal{I} \mathcal{A}$ be a NIA for a relation $\mathcal{R}$, with label set $\Lambda$, and let $\Phi$ be a set of label transformations. We say that $\mathcal{N} \mathcal{I} \mathcal{A}$ is $\Phi$-malleable label simulation extractable ( $\Phi$-ml-SE for short) if the following holds.

(i) There exists a PPT algorithm $\mathrm{S}_{0}$ that outputs a CRS $\omega$, a simulation trapdoor $\tau_{\text {sim }}$, and an extraction trapdoor $\tau_{\text {ext }}$.

(ii) There exists a PPT algorithm $\mathrm{K}$ and a negligible function $\nu: \mathbb{N} \rightarrow[0,1]$ such that, for all PPT adversaries $A$, we have

$$
\mathbb{P}\left[\mathbf{G}_{\mathcal{N} \mathcal{I} \mathcal{A}, \mathrm{A}, \mathrm{K}}^{\operatorname{lm}-\mathrm{se}}(\kappa, \Phi)\right] \leq \nu(\kappa),
$$

where the game $\mathbf{G}_{\mathcal{N} \mathcal{I} \mathcal{I}, \mathrm{A}, \mathrm{K}}^{\operatorname{lm}-\mathrm{s}}(\kappa, \Phi)$ is described in Fig. 1.

The last property, called label derivation privacy, says that it is hard to distinguish a fresh proof for some statement $x$ (with witness $w$ ) under label $\lambda$, from a proof re-randomized using algorithm LEval w.r.t. some function $\phi \in \Phi$; moreover, the latter should hold even if $(x, w, \lambda, \phi)$ are chosen adversarially (possibly depending on the CRS).

Definition 4 (Label derivation privacy). Let $\mathcal{N} \mathcal{I} \mathcal{A}$ be a NIA for a relation $\mathcal{R}$, and let $\Phi$ be a set of label transformations. We say that $\mathcal{N} \mathcal{I} \mathcal{A}$ has label derivation privacy if for all PPT A there exists a negligible function $\nu: \mathbb{N} \rightarrow[0,1]$ such that

$$
\left|\mathbb{P}\left[\mathbf{G}_{\mathcal{N} \mathcal{I} \mathcal{A}, \mathrm{A}}^{\mathrm{lb}-\text { prv }}(\kappa)=1\right]-\frac{1}{2}\right| \leq \nu(\kappa),
$$

where game $\mathbf{G}_{\mathcal{N} \mathcal{I} \mathcal{A}, \mathrm{A}}^{\text {lb-prv }}(\kappa)$ is described in Fig. 1.

\subsection{Public-Key Encryption}

A public-key encryption (PKE) scheme is a tuple of algorithms $\mathcal{P} \mathcal{K} \mathcal{E}=$ (Setup, KGen, Enc, Dec) defined as follows. (1) The (randomized) algorithm Setup takes as input the security parameter $1^{\kappa}$ and outputs public parameters $\rho \in\{0,1\}^{*}$; all algorithms are implicitly given $\rho$ as input. (2) The (randomized) algorithm KGen takes as input the public parameters $\rho$ and outputs a public/secret key pair $(p k, s k)$; the set of all secret keys is denoted by $\mathcal{S K}$, and the set of all public keys by $\mathcal{P K}$. Additionally, we require the existence of a (randomized) function PK which, upon input $s k \in \mathcal{S K}$, produces a valid public key $p k \in \mathcal{P K}$. (3) The (randomized) algorithm Enc takes as input the public key $p k$, a message $m \in \mathcal{M}$, and randomness $r \in\{0,1\}^{*}$, and outputs a ciphertext $c=\operatorname{Enc}(p k, m ; r)$; the set of all ciphertexts is denoted by $\mathcal{C}$. (4) The (deterministic) algorithm Dec takes as input the secret key $s k$ and a ciphertext $c$, and outputs $m=\operatorname{Dec}(s k, c)$ which is either equal to some message $m \in \mathcal{M}$, or to an error symbol $\perp$. We will require two additional algorithms, the first one to re-randomize a given ciphertext, and the second one for re-randomizing the secret key (without changing the public key). More formally: (5) The (randomized) algorithm UpdateC takes as input a ciphertext $c$, and outputs a new ciphertext $c^{\prime}$. (6) The (randomized) algorithm UpdateS takes as input a secret key $s k$, and outputs a new secret key $s k^{\prime}$.

We say that $\mathcal{P} \mathcal{K} \mathcal{E}$ satisfies correctness if for all $\rho \leftarrow \operatorname{Setup}\left(1^{\kappa}\right)$ and for all $(p k, s k) \leftarrow \operatorname{KGen}(\rho)$, we have that $\mathbb{P}[\operatorname{Dec}(\operatorname{UpdateS}(s k), \operatorname{UpdateC}(\operatorname{Enc}(p k, m)))=m]=1$, where the randomness is taken over the internal coin tosses of algorithms Enc, UpdateS and UpdateC. Additionally, we require that for all $\rho$ output by Setup, and for all $(p k, s k) \leftarrow \operatorname{KGen}(\rho)$ : (i) For any $s k^{\prime}$ such that $\operatorname{PK}\left(s k^{\prime}\right)=p k$, and any $c \in \mathcal{C}$, we have that $\operatorname{Dec}(s k, c)=\operatorname{Dec}\left(s k^{\prime}, c\right)$; (ii) For any $s k^{\prime} \leftarrow \operatorname{UpdateS}(s k)$, we have that $\mathrm{PK}(s k)=\mathrm{PK}\left(s k^{\prime}\right)$. 


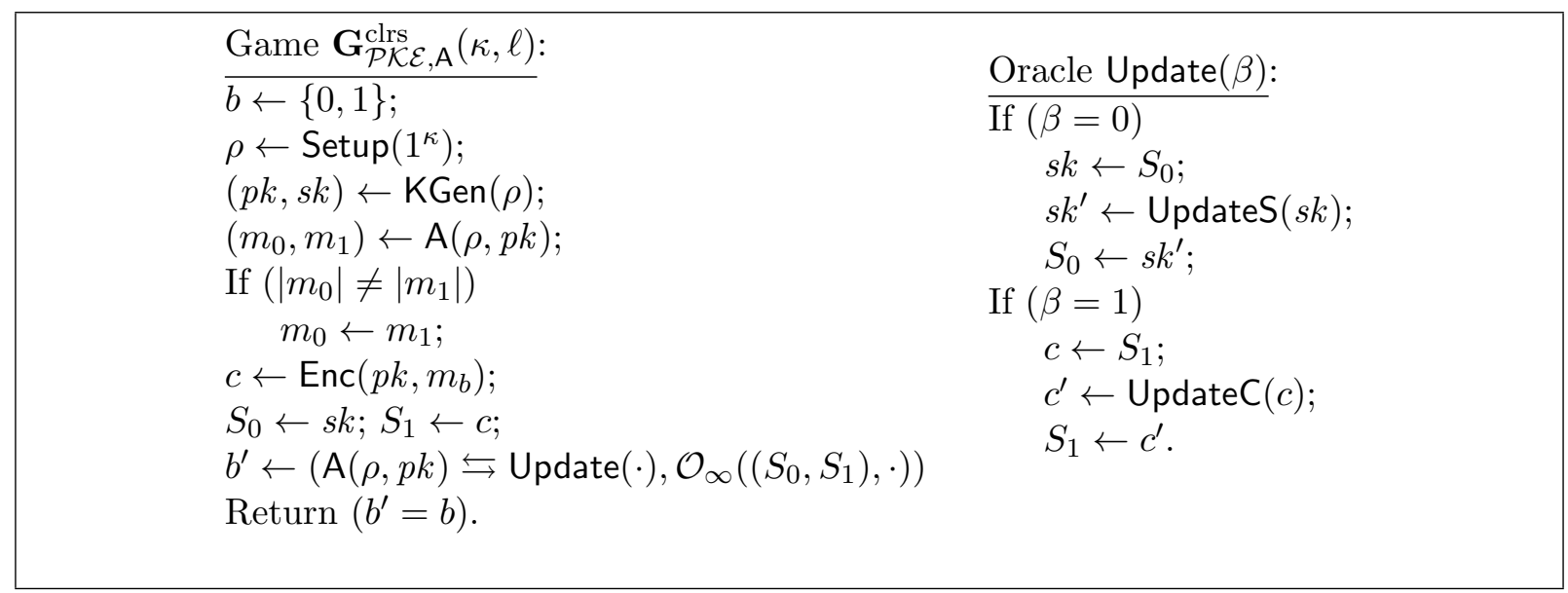

Figure 2: Game defining CLRS security of a PKE scheme.

We now turn to define continual leakage-resilient storage (CLRS) friendly PKE. Consider the game $\mathbf{G}_{\mathcal{P} \mathcal{K} E, A}^{\text {clrs }}(\kappa, \ell)$ as defined in Fig. 2. The definition below roughly say that a PKE scheme satisfies semantic security against all $\ell$-valid efficient adversaries that can observe independent leakage from $S_{0}=s k$ and $S_{1}=c$ (where $c$ is the challenge ciphertext).

Definition 5 (CLRS friendly PKE). For $\kappa \in \mathbb{N}$, let $\ell=\ell(\kappa)$ be the leakage parameter. We say that $\mathcal{P K \mathcal { E }}=$ (Setup, KGen, Enc, Dec, UpdateC, UpdateS) is $\ell$-noisy CLRS friendly if for all PPT $\ell$-valid adversaries $\mathrm{A}$ there exists a negligible function $\nu: \mathbb{N} \rightarrow[0,1]$ such that

$$
\left|\mathbb{P}\left[\mathbf{G}_{\mathcal{P} \mathcal{K}, \mathbf{A}}^{\mathrm{clrs}}(\kappa, \ell)=1\right]-\frac{1}{2}\right| \leq \nu(\kappa),
$$

where the game $\mathbf{G}_{\mathcal{P} \mathcal{K} \mathcal{E}, \mathbf{A}}^{\mathrm{clrs}}(\kappa, \ell)$ is described in Fig. 2.

We observe that Definition 5 is slightly different w.r.t. the definition of Dodis et al. [29]. In fact, on the one hand, our definition does not consider leakage from the update process, but, on the other hand, we consider noisy leakage and not just bounded leakage as in [29].

Finally, we introduce two extra properties relative to the algorithms UpdateC and UpdateS of a PKE scheme. The first property says that the distributions of fresh and updated ciphertexts are the same. The second property formalizes a similar requirement for fresh and updated keys.

Definition 6 (Ciphertext-update privacy). We say that $\mathcal{P K \mathcal { E }}$ is perfectly chipertext-update private if for all $\kappa \in \mathbb{N}$, all $\rho \leftarrow \operatorname{Setup}\left(1^{\kappa}\right)$, all $(p k, s k) \leftarrow \mathrm{KGen}(\rho)$, and any $m \in \mathcal{M}, c \in$ $\mathcal{C}$ such that $c=\operatorname{Enc}(p k, m ; r)$ for some $r \in\{0,1\}^{*}$, the distributions $\{p k$, Enc $(p k, m)\}$ and $\{p k$, UpdateC $(c)\}$ are equivalent.

Definition 7 (Secret-key-update privacy). We say that $\mathcal{P K \mathcal { E }}$ is perfectly secret-key-update private if for all $\kappa \in \mathbb{N}$, all $\rho \leftarrow \operatorname{Setup}\left(1^{\kappa}\right)$, and all $(p k, s k) \leftarrow \operatorname{KGen}(\rho)$, the distributions $\{p k$, UpdateS $(s k)\}$ and $\left\{\left(p k^{\prime}, s k^{\prime}\right) \leftarrow \operatorname{KGen}(\rho): p k^{\prime}=p k\right\}$ are equivalent.

\subsection{Commitment Schemes}

A (non-interactive) commitment scheme with message space $\mathcal{M}$ is a tuple of algorithms $\mathcal{C O} \mathcal{M}=$ (CRSGen, Commit) defined as follow: (1) The (randomized) algorithm CRSGen takes as input the security parameter $1^{\kappa}$, and outputs a commitment key $\omega ;(2)$ The (randomized) algorithm 


\begin{tabular}{|c|c|}
\hline $\begin{array}{l}\text { Experiment } \mathbf{G}_{\mathcal{S} \mathcal{S}, \mathrm{A}}^{\text {euf-cma }}(\kappa) \text { : } \\
\mathcal{Q} \leftarrow \emptyset ; \rho \leftarrow \operatorname{Setup}\left(1^{\kappa}\right) \\
(v k, s k) \leftarrow \operatorname{Gen}(\rho) ; \\
\left(m^{*}, \sigma^{*}\right) \leftarrow\left(\mathrm{A}(v k, \rho) \leftrightarrows \mathcal{O}_{\text {sign }}(s k, \cdot)\right) \\
\text { Return } 1 \text { iff: } \\
\quad \text { (i) } m^{*} \notin \mathcal{Q} \\
\quad \text { (ii) } \operatorname{Vrfy}\left(v k,\left(m^{*}, \sigma^{*}\right)=1\right)\end{array}$ & $\begin{array}{l}\text { Oracle } \mathcal{O}_{\text {sign }}(s k, m): \\
\mathcal{Q} \leftarrow \mathcal{Q} \cup\{m\} ; \\
\text { Return } \sigma \leftarrow \operatorname{Sign}(s k, m) . \\
\text { Experiment } \mathbf{G}_{\mathcal{C O} \mathcal{M}, \mathrm{A}}^{\text {hide }}(\kappa, b): \\
\omega \leftarrow \operatorname{CRSGen}\left(1^{\kappa}\right) ; \\
\left(m_{0}, m_{1}\right) \leftarrow \mathrm{A}(\omega) ; \\
\gamma \leftarrow \operatorname{Commit}\left(\omega, m_{b}\right) ; \\
\text { Return } \mathrm{A}(\omega, \gamma) .\end{array}$ \\
\hline
\end{tabular}

Figure 3: Games defining computational hiding of a commitment scheme, and universal unforgeability against chosen-message attacks of a signature scheme.

Commit takes as input the commitment key $\omega$, a message $m \in \mathcal{M}$, and randomness $r \in\{0,1\}^{*}$, and outputs a commitment $\gamma$.

Intuitively a commitment scheme satisfies two properties. The first property, called the hiding property, says that the commitment hides the message; the second property, called the binding property says that it is hard to open a valid commitment in two different ways. Below, we define these properties directly in the flavor we need for our application.

Definition 8 (Hiding and binding). Let $\mathcal{C O} \mathcal{M}=$ (CRSGen, Commit) be a commitment scheme. We say that $\mathcal{C O M}$ is:

- Computationally hiding, if for all PPT adversaries A there exists a negligible function $\nu: \mathbb{N} \rightarrow[0,1]$ such that

$$
|| \mathbb{P}\left[\mathbf{G}_{\mathcal{C O M}, \mathbf{A}}^{\text {hide }}(\kappa, 0)=1\right]-\mathbb{P}\left[\mathbf{G}_{\mathcal{C O} \mathcal{M}, \mathrm{A}}^{\text {hide }}(\kappa, 1)=1\right] \mid \leq \nu(\kappa),
$$

where the game $\mathbf{G}_{\mathcal{C O M}, \mathrm{A}}^{\text {hide }}$ is described in Fig. 3.

- Statistically binding if for all even unbounded adversaries A there exists a negligible function $\nu: \mathbb{N} \rightarrow[0,1]$ such that:

$$
\mathbb{P}\left[\operatorname{Commit}(\omega, m ; r)=\operatorname{Commit}\left(\omega, m^{\prime} ; r^{\prime}\right) \wedge m \neq m^{\prime}: \quad \begin{array}{c}
\omega \leftarrow \operatorname{CRSGen}\left(1^{\kappa}\right) ; \\
\left(m, r, m^{\prime}, r^{\prime}\right) \leftarrow \mathrm{A}(\omega)
\end{array}\right] \leq \nu(\kappa) .
$$

\subsection{Signature Schemes}

A signature scheme is a tuple of algorithms $\mathcal{S S}=$ (Setup, KGen, Sign, Vrfy) defined as follow: (1) The (randomized) algorithm Setup takes as input the security parameter $1^{\kappa}$ and outputs public parameters $\rho \in\{0,1\}^{*}$; all algorithms are implicitly given $\rho$ as input. (2) The (randomized) algorithm KGen takes as input the public parameters and outputs a verification/signing key pair $(v k, s k)$. (3) The (randomized) algorithm Sign takes as input the secret key $s k$ and a message $m$, and outputs a signature $\sigma$. (4) The (deterministic) algorithm Vrfy takes as input the verification key $v k$ and a pair $(m, \sigma)$ and outputs a decision bit.

For completeness, we require that for all $\rho$ output by Setup, and for all $(v k, s k)$ output by $\operatorname{KGen}(\rho)$, and for any message $m$, we have that $\operatorname{Vrfy}(v k$, Sign $(s k, m))=1$ with probability one over coin tosses of the signing algorithm. As for security, we require that no efficient adversary with oracle access to a signature oracle and given the verification key, can forge a valid signature on a fresh message (i.e., a message not returned by the signature oracle). 


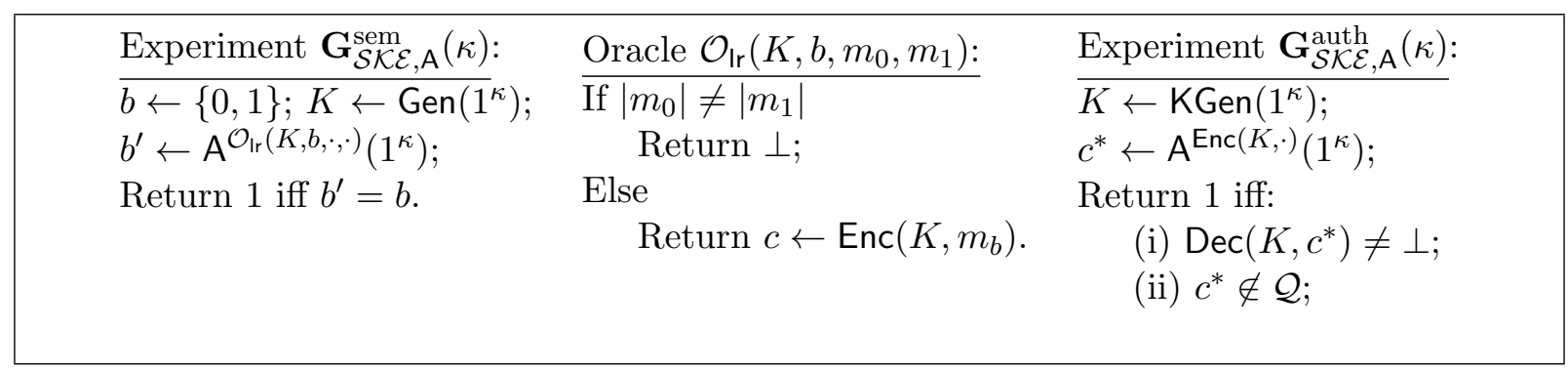

Figure 4: Games defining semantic security and authenticity of an SKE scheme; the set $\mathcal{Q}$ in the last game contains all ciphertexts returned by oracle $\operatorname{Enc}(K, \cdot)$.

Definition 9 (EUF-CMA security). Let $\mathcal{S S}=$ (Setup, KGen, Sign, Vrfy) be a signature scheme. We say that $\mathcal{S S}$ is existentially unforgeable against chosen-message attacks (EUF-CMA) if for all PPT adversaries $\mathrm{A}$ there exists a negligible function $\nu: \mathbb{N} \rightarrow[0,1]$ such that

$$
\mathbb{P}\left[\mathbf{G}_{\mathcal{S} S, A}^{\text {euf-cma }}(\kappa)=1\right] \leq \nu(\kappa),
$$

where the game $\mathbf{G}_{\mathcal{S} \mathcal{S}, \mathbf{A}}^{\text {euf }}(\kappa)$ is described in Fig. 3.

\subsection{Authenticated Encryption}

A secret key encryption (SKE) scheme consists of three PPT algorithms $\mathcal{S K \mathcal { E }}:=($ KGen, Enc, Dec) specified as follows. (1) The (randomized) key generation algorithm KGen takes as input the security parameter $1^{\kappa}$, and outputs a key $K ;(2)$ The (randomized) encryption algorithm Enc takes as input a key $K$, a message $m \in\{0,1\}^{*}$, random coins $r \in\{0,1\}^{*}$, and outputs a ciphertext $c$; (3) The (deterministic) decryption algorithm Dec takes as input a key $K$ and a ciphertext $c$, and outputs a message $m$ or a special symbol $\perp$ (indicating an error).

An SKE scheme meets correctness if for all keys $K$ output by $\mathrm{KGen}\left(1^{\kappa}\right)$, and for all messages $m \in\{0,1\}^{*}$, we have that $\operatorname{Dec}(K, \operatorname{Enc}(K, m))=m$ with probability one over the random coin tosses of the encryption algorithm. As for security, we define two properties that make SKE a so-called authenticated encryption scheme.

Let $\mathcal{O}_{\mathrm{Ir}}(K, b)$ be an oracle that, upon input two message $m_{0}, m_{1} \in\{0,1\}^{*}$, returns $c \leftarrow$ $\operatorname{Enc}\left(K, m_{b}\right)$ if $\left|m_{0}\right|=\left|m_{1}\right|$ (and $\perp$ otherwise). The first property says that it is hard to distinguish the encryption of two messages, even when given access to such an encryption oracle.

Definition 10. (Semantic security) An SKE scheme $\mathcal{S K \mathcal { E }}=$ (KGen, Enc, Dec) is semantically secure if for all PPT adversaries $\mathrm{A}$ there is a negligible function $\nu: \mathbb{N} \rightarrow[0,1]$ such that

$$
\left|\mathbb{P}\left[\mathbf{G}_{\mathcal{S} K \mathcal{E}, \mathbf{A}}^{\mathrm{sem}}(\kappa)=1\right]-\frac{1}{2}\right| \leq \nu(\kappa),
$$

where the game $\mathbf{G}_{\mathcal{S} \mathcal{K} \text {, } \mathrm{A}}^{\mathrm{sem}}(\kappa)$ is described in Fig. 4.

The second property says that SKE has an elusive range, meaning that, without knowing the secret key, it is hard to produce a valid ciphertext.

Definition 11. (Authenticity) An SKE scheme $\mathcal{S K E}=($ KGen, Enc, Dec) satisfies authenticity if for all PPT adversaries $\mathrm{A}$ there is a negligible function $\nu: \mathbb{N} \rightarrow[0,1]$ such that

$$
\mathbb{P}\left[\mathbf{G}_{\mathcal{S} \mathcal{K} \mathcal{E}, \mathrm{A}}^{\text {auth }}(\kappa)=1\right] \leq \nu(\kappa),
$$

where the game $\mathbf{G}_{\mathcal{S} \mathcal{K} \mathcal{E}, \mathrm{A}}^{\text {auth }}(\kappa)$ is described in Fig. 4. 


\section{Non-Malleability with Refresh}

\subsection{Split-State Codes}

A coding scheme in the CRS model is a tuple of polynomial-time algorithms $\mathcal{C S}=($ Init, Encode, Decode) with the following syntax: (1) The (randomized) initialization algorithm Init takes as input the security parameter $1^{\kappa}$, and outputs a CRS $\omega \in\{0,1\}^{*} ;(2)$ The (randomized) encoding algorithm Encode takes as input the CRS $\omega$ and a message $M \in \mathcal{M}$, and outputs a codeword $C \in \mathcal{C}$; (3) The (deterministic) decoding algorithm Decode takes as input the CRS $\omega$ and a codeword $C \in \mathcal{C}$, and outputs a value $M \in \mathcal{M} \cup\{\perp\}$ (where $\perp$ denotes an invalid codeword). A coding scheme is correct if for all $\omega$ output by $\operatorname{Init}\left(1^{\kappa}\right)$, and any $M \in \mathcal{M}$, we have $\mathbb{P}[\operatorname{Decode}(\omega, \operatorname{Encode}(\omega, M))=M]=1$, where the probability is taken over the randomness of the encoding algorithm.

We consider coding schemes with an efficient refreshing algorithm. Specifically, for a coding scheme $\mathcal{C S}$ we assume there exists a randomized algorithm Rfrsh that, upon input the CRS $\omega$ and a codeword $C \in \mathcal{C}$, outputs a codeword $C^{\prime} \in \mathcal{C}$. For correctness we require that for all $\omega$ output by $\operatorname{Init}\left(1^{\kappa}\right)$, we have $\mathbb{P}[\operatorname{Decode}(\omega, \operatorname{Rfrsh}(\omega, C))=\operatorname{Decode}(\omega, C)]=1$, where the probability is over the randomness used by the encoding and refreshing algorithms.

Split-state model. In this paper we are interested in coding schemes in the split-state model, where a codeword consists of two parts that can be refreshed independently and without the need of any interaction. More precisely, given a codeword $C:=\left(C_{0}, C_{1}\right)$, the refresh procedure $\operatorname{Rfrsh}\left(\omega,\left(\beta, C_{\beta}\right)\right)$, for $\beta \in\{0,1\}$, takes as input either the left or the right part of the codeword, and updates it. Sometimes we also write $\operatorname{Rfrsh}(\omega, C)$ as a shorthand for the algorithm that independently executes $\operatorname{Rfrsh}\left(\omega,\left(0, C_{0}\right)\right)$ and $\operatorname{Rfrsh}\left(\omega,\left(1, C_{1}\right)\right)$.

Correctness here means that for all $\omega$ output by $\operatorname{Init}\left(1^{\kappa}\right)$, for all $C \in \mathcal{C}$, and for any $\beta \in$ $\{0,1\}$, if we let $C^{\prime}=\left(C_{0}^{\prime}, C_{1}^{\prime}\right)$ be such that $C_{\beta}^{\prime} \leftarrow \operatorname{Rfrsh}\left(\omega,\left(\beta, C_{\beta}\right)\right)$ and $C_{1-\beta}^{\prime}=C_{1-\beta}$, then $\mathbb{P}\left[\operatorname{Decode}\left(\omega, C^{\prime}\right)=\operatorname{Decode}(\omega, C)\right]=1$.

\subsection{The Definition}

We give the security definition for continuously non-malleable codes with split-state refresh (R-CNMCs for short). Our notion compares two experiments, which we denote by Tamper and SimTamper (cf. Fig. 5). Intuitively, in the experiment Tamper we consider an adversary continuously tampering with, and leaking from, a target encoding $C=\left(C_{0}, C_{1}\right)$ of a message $M \in \mathcal{M}$ (the message can be chosen adaptively, depending on the CRS). For each tampering attempt $\left(f_{0}, f_{1}\right)$, the adversary gets to see the output $\widetilde{M}$ of the decoding corresponding to the modified codeword $\widetilde{C}=\left(f_{0}\left(C_{0}\right), f_{1}\left(C_{1}\right)\right)$. Tampering is non-persistent, meaning that each tampering function is applied to the original codeword $C$, until, eventually, a decoding error happens; after that, the target encoding $C$ is refreshed, and the adversary can start tampering with, and leaking from, the refreshed codeword.

In the experiment SimTamper, we consider a simulator $S=\left(S_{0}, S_{1}\right)$, where $S_{0}$ outputs a simulated CRS, while $S_{1}$ 's goal is to simulate the view of the adversary in the real experiment; the simulator $\mathrm{S}_{1}$, in faking a tampering query $\left(f_{0}, f_{1}\right)$, is allowed to output a special value $\diamond$, signaling that (it believes) the adversary did not change the encoded message, in which case the experiment replaces $\diamond$ with $M$. We stress that the simulator $\mathrm{S}$ is stateful; in particular algorithms $\mathrm{S}_{0}, \mathrm{~S}_{1}$ implicitly share a state.

For technical reasons, in some of our applications, we will actually need a slightly stronger security property, where after a decoding error happens, and before the original codeword is refreshed, the adversary is allowed to make one extra tampering query $\left(f_{0}^{*}, f_{1}^{*}\right)$. Hence, if the 


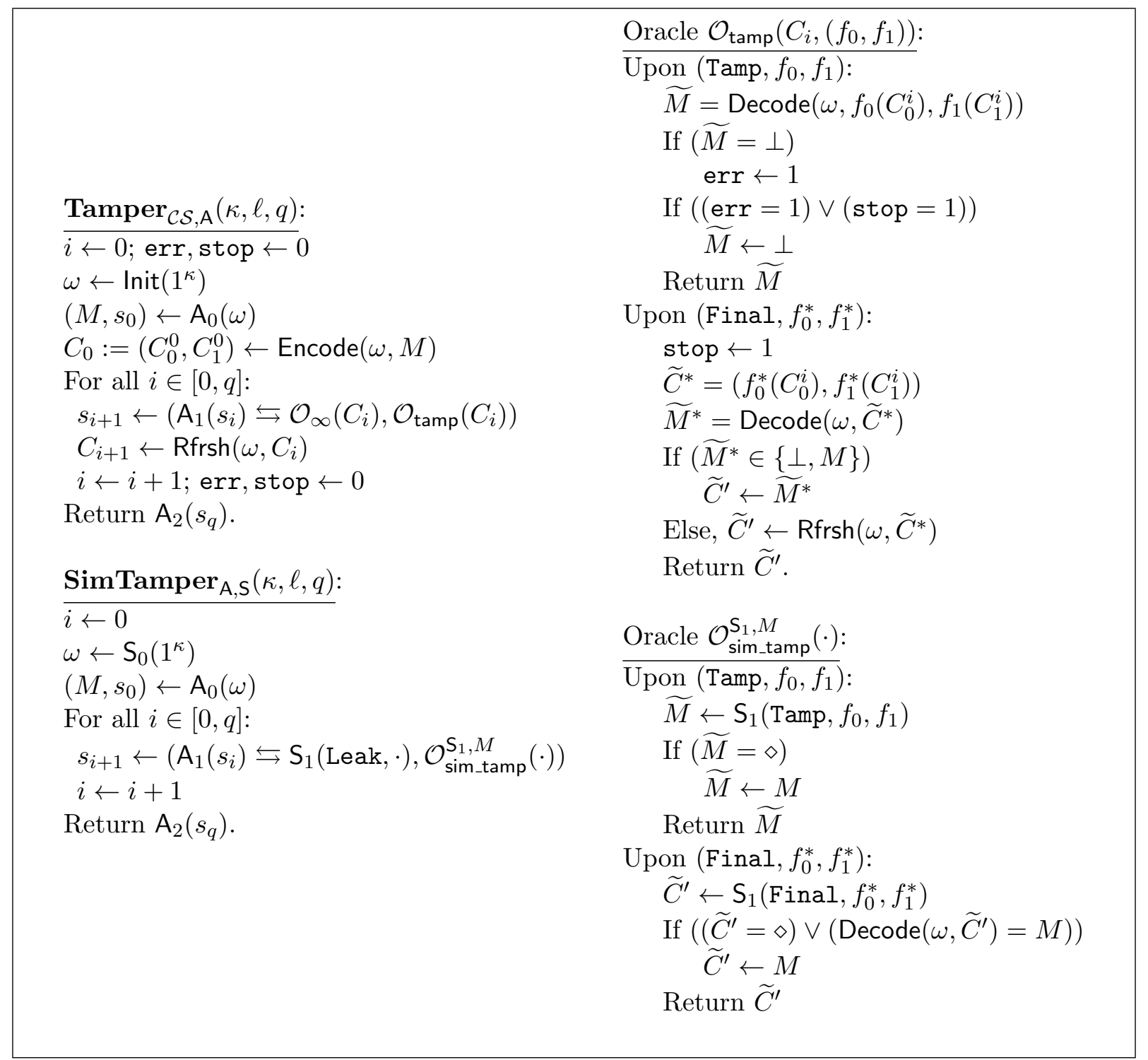

Figure 5: Experiments defining continuously non-malleable codes with split-state refresh.

corresponding tampered codeword $\widetilde{C}^{*}$ is valid and is not an encoding of the original message $M$, the attacker receives a refreshing of $\widetilde{C}^{*}$; otherwise it obtains either $M$ (in the former case) or $\perp$ (in the latter case). Unfortunately, it is impossible to further generalize our definition to handle the refreshing of invalid codewords. ${ }^{7}$

Definition 12 (Continuous non-malleability with split-state refresh). For $\kappa \in \mathbb{N}$, let $\ell=\ell(\kappa)$ be a parameter. We say that a coding scheme $\mathcal{C S}$ is an $\ell$-leakage-resilient and continuously non-malleable code with split-state refresh (R-CNMC for short) if for all adversaries $A:=$ $\left(A_{0}, A_{1}, A_{2}\right)$, where $A_{0}$ and $A_{2}$ are PPT algorithms and $A_{1}$ is an $\ell$-valid (cf. Section 2.1) deterministic polynomial-time algorithm, there exists a PPT simulator $S=\left(S_{0}, S_{1}\right)$ and a negligible

\footnotetext{
${ }^{7}$ This can be seen by the following attack. Consider an adversary that computes offline a valid codeword $\left(\bar{C}_{0}, \bar{C}_{1}\right)$, and then makes two extra tampering queries (at the end of two subsequent rounds, say, $i$ and $i+1$ ) such that the first query overwrites $\left(C_{0}^{i}, C_{1}^{i}\right)$ with $\left(C_{0}^{i}, \bar{C}_{1}\right)$, and the second query overwrites $\left(C_{0}^{i+1}, C_{1}^{i+1}\right)$ with $\left(\bar{C}_{0}, C_{1}^{i+1}\right)$; by combining the refreshed codewords obtained as output, the adversary gets a refreshing of the original codeword, which cannot be simulated in the ideal experiment (recall that the refresh algorithm updates the two shares independently).
} 
function $\nu: \mathbb{N} \rightarrow[0,1]$ such that, for any polynomial $q(\kappa)$, the following holds:

$$
\left|\mathbb{P}\left[\operatorname{Tamper}_{\mathcal{C S}, \mathrm{A}}(\kappa, \ell, q)=1\right]-\mathbb{P}\left[\operatorname{SimTamper}_{\mathrm{A}, \mathrm{S}}(\kappa, \ell, q)=1\right]\right| \leq \nu(\kappa),
$$

where the experiments $\operatorname{Tamper}_{\mathcal{C S}, \mathrm{A}}(\kappa, \ell, q)$ and $\mathbf{S i m T a m p e r}_{\mathrm{A}, \mathrm{S}}(\kappa, \ell, q)$ are defined in Fig. 5 .

We note that in the Tamper security game the adversary does not have "direct" access to a refreshing oracle (namely, an oracle that, upon request, would refresh the codeword). This choice is without loss of any generality, as a call to the refreshing oracle can always be simulated by a tampering query yielding an invalid codeword (which indeed triggers a refreshing in our experiment).

\section{Code Construction}

Let $\mathcal{P K \mathcal { E }}=($ Setup, KGen, Enc, Dec, UpdateC, UpdateS) be a CLRS friendly PKE scheme, with secret-key space $\mathcal{S K}$. Let $\mathcal{C O M}=($ CRSGen, Commit) be a commitment scheme in the CRS model. Consider the following NP-relations, parameterized by the PKE and the commitment scheme, respectively:

$$
\begin{aligned}
& \mathcal{R}_{0}:=\{(p k, s k): p k=\operatorname{PK}(s k), s k \in \mathcal{S} \mathcal{K}\} \\
& \mathcal{R}_{1}:=\{((\omega, \gamma),(M, r)): \gamma=\operatorname{Commit}(\omega, M ; r)\} .
\end{aligned}
$$

Let $\Phi_{0}$ and $\Phi_{1}$ be two sets of label transformations defined below:

$$
\begin{aligned}
& \Phi_{0}:=\{\phi: \exists p k, s k \text { s.t. }(\forall m, r) \operatorname{Dec}(s k, \phi(\operatorname{Enc}(p k, m ; r)))=m, p k=\operatorname{PK}(s k)\} \\
& \Phi_{1}:=\{\phi:(\forall s k) \operatorname{PK}(s k)=\operatorname{PK}(\phi(s k))\} .
\end{aligned}
$$

Notice that $\mathcal{R}_{0}, \mathcal{R}_{1}, \Phi_{0}$ and $\Phi_{1}$ are implicitly parameterized by the public parameters $\rho \in\{0,1\}^{*}$ of the PKE scheme. Finally, let $\mathcal{U}^{0}$ and $\mathcal{U}_{1}$ be the following sets of label transformations:

$$
\begin{aligned}
& \mathcal{U}^{0}:=\left\{\operatorname{UpdateC}\left(\cdot ; r_{u}\right): r_{u} \in\{0,1\}^{*}\right\} \\
& \mathcal{U}^{1}:=\left\{\operatorname{UpdateS}\left(\cdot ; r_{u}\right): r_{u} \in\{0,1\}^{*}\right\} .
\end{aligned}
$$

It is easy to verify that $\mathcal{U}_{\beta} \subseteq \Phi_{\beta}$, for $\beta \in\{0,1\}$. In fact, for $\beta=0$, by the correctness of the PKE scheme, there exists $s k$ such that $\mathbb{P}[\operatorname{Dec}(s k, \operatorname{UpdateC}(\operatorname{Enc}(p k, m)))=m]=1$ and $p k=\operatorname{PK}(s k)$; similarly, for $\beta=1$, again by correctness of the PKE scheme, for any $s k^{\prime} \leftarrow \operatorname{UpdateS}(p k, s k)$ we have that $\mathrm{PK}(s k)=\mathrm{PK}\left(s k^{\prime}\right)$.

Scheme description. Let $\mathcal{N} \mathcal{I}_{\mathcal{A}_{0}}=\left(\mathrm{CRSGen}_{0}\right.$, Prove $\left._{0}, \mathrm{Vrfy}_{0}, \mathrm{LEval}_{0}\right)$ and $\mathcal{N} \mathcal{I}_{\mathcal{A}_{1}}=\left(\mathrm{CRSGen}_{1}\right.$, Prove $_{1}, \mathrm{Vrfy}_{1}, \mathrm{LEval}_{1}$ ) be NIAs for the above defined relations $\mathcal{R}_{0}$ and $\mathcal{R}_{1}$. Our code $\mathcal{C} \mathcal{S}=$ (Init, Encode, Decode) works as follows.

- Init $\left(1^{\kappa}\right)$ : For $\beta \in\{0,1\}$, sample $\omega_{\beta} \leftarrow \operatorname{CRSGen}_{\beta}\left(1^{\kappa}\right), \omega \leftarrow \operatorname{CRSGen}\left(1^{\kappa}\right)$, and $\rho \leftarrow \operatorname{Setup}\left(1^{\kappa}\right)$. $\overline{\operatorname{Return}} \bar{\omega}=\left(\omega_{0}, \omega_{1}, \omega, \rho\right)$.

- Encode $(\bar{\omega}, M)$ : Parse $\bar{\omega}:=\left(\omega_{0}, \omega_{1}, \omega, \rho\right)$, sample $(p k, s k) \leftarrow \operatorname{KGen}(\rho)$, and $r \leftarrow\{0,1\}^{*}$. Compute $c \leftarrow \operatorname{Enc}(p k, M \| r), \gamma=\operatorname{Commit}(\omega, M ; r)$, and $\pi_{0} \leftarrow \operatorname{Prove}_{0}^{c}\left(\omega_{0}, p k, s k\right)$, and $\pi_{1} \leftarrow \operatorname{Prove}_{1}^{s k}\left(\omega_{1},(\omega, \gamma),(M, r)\right)$. Set $C_{0}:=\left(p k, c, \pi_{0}\right)$ and $C_{1}:=\left(s k, \gamma, \pi_{1}\right)$, and return $C:=\left(C_{0}, C_{1}\right)$.

- Decode $(\bar{\omega}, C)$ : Parse $\bar{\omega}:=\left(\omega_{0}, \omega_{1}, \omega, \rho\right)$ and $C:=\left(C_{0}, C_{1}\right)$, where $C_{1}:=\left(s k, \gamma, \pi_{1}\right)$ and $\left.\overline{C_{0}=\left(p k, c, \pi_{0}\right.}\right)$. Compute $M \| r:=\operatorname{Dec}(s k, c)$, and if the following conditions hold return $M$ else return $\perp$ : 
I. Left check: $\operatorname{Ver}_{0}^{c}\left(\omega_{0}, p k, \pi_{0}\right)=1$.

II. Right check: $\operatorname{Ver}_{1}^{s k}\left(\omega_{1},(\omega, \gamma), \pi_{1}\right)=1$.

III. Cross check: $\operatorname{Commit}(\omega, M ; r)=\gamma$.

- $\operatorname{Rfrsh}\left(\bar{\omega},\left(\beta, C_{\beta}\right)\right):$ Parse $\bar{\omega}:=\left(\omega_{0}, \omega_{1}, \omega, \rho\right), C_{0}:=\left(p k, c, \pi_{0}\right)$, and $C_{1}=\left(s k, \gamma, \pi_{1}\right)$. Hence:

- For $\beta=0$, pick $r_{\text {upd }}^{0} \leftarrow\{0,1\}^{*}$, let $c^{\prime}:=\operatorname{UpdateC}\left(c ; r_{\text {upd }}^{0}\right)$ and $\pi_{0}^{\prime} \leftarrow \operatorname{LEval}_{0}\left(\omega_{0}\right.$, $\left.\operatorname{UpdateC}\left(\cdot ; r_{\text {upd }}^{0}\right),\left(p k, c, \pi_{0}\right)\right)$, and return $C_{0}^{\prime}:=\left(p k, c^{\prime}, \pi_{0}^{\prime}\right)$.

- For $\beta=1$, pick $r_{\text {upd }}^{1} \leftarrow\{0,1\}^{*}$, let $s k^{\prime}:=\operatorname{UpdateS}\left(s k ; r_{\text {upd }}^{1}\right)$, and $\pi_{1}^{\prime} \leftarrow \operatorname{LEval}_{1}\left(\omega_{1}\right.$, UpdateS $\left.\left(\cdot ; r_{\text {upd }}^{1}\right),\left((\gamma, \omega), s k, \pi_{1}\right)\right)$, and return $C_{1}^{\prime}:=\left(\gamma, s k^{\prime}, \pi_{1}^{\prime}\right)$.

Theorem 1. Let $\mathcal{P K \mathcal { E }}$ be a PKE scheme with message space $\mathcal{M}_{\text {pke }}$ and public-key space $\mathcal{P K}$, let $\mathcal{C O M}$ be a commitment scheme with message space $\mathcal{M}$, and let $\mathcal{N} \mathcal{I} \mathcal{A}_{0}$ (resp. $\mathcal{N} \mathcal{I} \mathcal{A}_{1}$ ) be a $N I A$ w.r.t. the relations $\mathcal{R}_{0}\left(\right.$ resp. $\left.\mathcal{R}_{1}\right)$. Define $\mu(\kappa):=\log |\mathcal{M}|, \mu_{\mathrm{pke}}(\kappa):=\log \left|\mathcal{M}_{\text {pke }}\right|$, and $\delta(\kappa):=\log |\mathcal{P K}|$.

For any $\ell \in \mathbb{N}$, assuming that $\mathcal{P K \mathcal { E }}$ is an $\left(\ell+3 \mu+2 \kappa+\max \left\{\delta, \mu_{\mathrm{pke}}\right\}\right)$-noisy CLRS-friendly $P K E$ scheme (cf. Definition 5), that $\mathcal{C O M}$ is a non-interactive statistically binding commitment scheme, and that $\mathcal{N} \mathcal{I} \mathcal{A}_{0}$ (resp. $\mathcal{N} \mathcal{I} \mathcal{A}_{1}$ ) satisfies adaptive multi-theorem zero-knowledge (cf. Definition 2), $\Phi_{0}$-malleable (resp. $\Phi_{1}$-malleable) label simulation extractability (cf. Definition 3), and label derivation privacy (cf. Definition 4), then the coding scheme $\mathcal{C S}$ described above is an

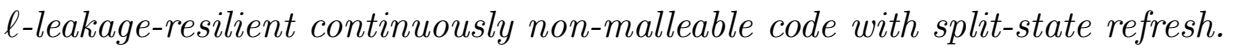

\subsection{Proof Intuition}

The proof of the above theorem is quite involved. We provide some highlights here. Consider a simulator $\left(\mathrm{S}_{0}, \mathrm{~S}_{1}\right)$, where $\mathrm{S}_{0}$ simulates a fake $\operatorname{CRS} \bar{\omega}=\left(\omega_{0}, \omega_{1}, \omega, \rho\right)$ by additionally sampling the corresponding zero-knowledge and extraction trapdoors for the NIAs (which are then passed to $\mathrm{S}_{1}$ ). At the core of our simulation strategy are two algorithms $\mathrm{T}^{0}$ and $\mathrm{T}^{1}$, whose goal is essentially to emulate the outcome of the real tampering experiment, with the important difference that $\mathrm{T}^{0}$ is only given the left part of a (simulated) codeword $C_{0}$ and the left tampering function $f_{0}$, whereas $\mathrm{T}^{1}$ is given $\left(C_{1}, f_{1}\right)$.

The simulator $\mathrm{S}_{1}$ then works as follows. Initially, it samples a fresh encoding $\left(C_{0}, C_{1}\right)$ of $0^{\mu}$. More in details, the fresh encoding comes from the (computationally close) distribution where the proofs $\pi_{0}$ and $\pi_{1}$ are simulated proofs. At the beginning of each round, it runs a simulated refresh procedure in which the ciphertext $c$ is updated via UpdateC (and the simulated proof $\pi_{0}$ is re-computed using fresh randomness), and similarly the secret key $s k$ is updated via UpdateS (and the simulated proof $\pi_{1}$ is re-computed using fresh randomness). Hence, for each tampering query $\left(f_{0}, f_{1}\right)$, the simulator $\mathrm{S}_{1}$ runs $\widetilde{M}_{0}:=\mathrm{T}^{0}\left(C_{0}, f_{0}\right), \widetilde{M}_{1}:=\mathrm{T}^{1}\left(C_{1}, f_{1}\right)$, and it returns $\widetilde{M}_{0}$ as long as $\perp \neq \widetilde{M}_{0}=\widetilde{M}_{1} \neq \perp$ (and $\perp$ otherwise). The extra tampering query $\left(f_{0}^{*}, f_{1}^{*}\right)$ is simulated similarly, based on the outcome of the tampering simulators $\left(\mathrm{T}^{0}, \mathrm{~T}^{1}\right)$. We briefly describe the tampering simulators $\mathrm{T}^{0}$ and $\mathrm{T}^{1}$ :

- Algorithm $\mathrm{T}^{0}$ lets $f_{0}\left(C_{0}\right):=\left(\widetilde{p k}, \widetilde{c}, \widetilde{\pi}_{0}\right)$. If the proof $\widetilde{\pi}_{0}$ does not verify, it returns $\perp$. Else, if $\left(\widetilde{p k}, \widetilde{c}, \widetilde{\pi}_{0}\right)=\left(p k, c, \pi_{0}\right)$, it returns $\diamond$. Else, it extracts the proof $\widetilde{\pi}_{0}$, this leads to two possible outcomes: ${ }^{8}$

(a) The extractor outputs a secret key $\widehat{s k}$ which is used to decrypt $\widetilde{c}$, and the tampering simulator returns the corresponding plaintext $\widetilde{M}$.

\footnotetext{
${ }^{8}$ The above description is simplified, in that extraction could potentially fail, however, this happens only with negligible probability when the proof verifies correctly.
} 
(b) The extractor outputs a transformation $\phi$ which maps the label of the simulated proof $\pi_{0}$, namely the encryption of $0^{\mu}$, to $\widetilde{c}$. In this case the tampering function $f_{0}$ has modified the original ciphertext $c$ to the mauled ciphertext $\widetilde{c}$ which is an encryption of the same message, so the tampering simulator returns $\diamond$.

- Algorithm $T^{1}$ lets $f_{1}\left(C_{1}\right):=\left(\widetilde{\gamma}, \widetilde{s k}, \widetilde{\pi}_{1}\right)$. If the proof $\widetilde{\pi}_{1}$ does not verify, it returns $\perp$. Else, if $\left(\widetilde{\gamma}, \widetilde{s k}, \widetilde{\pi}_{1}\right)=\left(\gamma, s k, \pi_{1}\right)$, it returns $\diamond$. Else, it extracts the proof $\widetilde{\pi}_{1}$, again, this leads to two possible outcomes:

(a) The extractor outputs the committed message $\widetilde{M}$ (along with the randomness of the commitment), so the tampering simulator can simply return $\widetilde{M}$.

(b) The extractor outputs a transformation $\phi$ which maps the label of the simulated proof $\pi_{1}$, namely the original secret key $s k$, to the mauled secret key $\widetilde{s k}$. In this case, the mauled proof $\widetilde{\pi}_{1}$ must be a valid proof whose instance is the original commitment, and so, once again, the tampering simulator returns $\diamond$.

To show that the above simulator indeed works, we use a hybrid argument where we incrementally change the distribution of the ideal tampering experiment until we reach the distribution of the real tampering experiment. Each step introduces a negligible error, thanks to the security properties of the underlying building blocks. Perhaps, the most interesting step is the one where we switch the ciphertext $c$ from an encryption of zero to an encryption of the real message (to which we always have to append the randomness of the commitment); in order to show that this change is unnoticeable, we rely on the CLRS storage friendly security of the PKE scheme. In particular, this step of the proof is based on the following observations:

- The reduction can perfectly emulate the distribution of the CRS $\bar{\omega}$, and of all the elements $\left(p k, \pi_{0}, \gamma, \pi_{1}\right)$, except for $(c, s k)$. However, by outputting $\left(0^{\mu}\|r, M\| r\right)$ as challenge plaintexts - where $r \in\{0,1\}^{*}$ is the randomness for the commitment-the reduction can obtain independent leakages from $C_{0}$ and $C_{1}$ with the right distribution.

- Refresh of codewords can also be emulated by exploiting the fact that the reduction is allowed to update the challenge secret key and ciphertext.

- The reduction can answer tampering queries from the adversary by using $\mathrm{T}^{0}$ and $\mathrm{T}^{1}$ as leakage functions. The main obstacle is to ensure that $\mathrm{T}^{0}$ and $\mathrm{T}^{1}$ are $\ell$-leaky, where $\ell \in \mathbb{N}$ is the leakage bound tolerated by the PKE scheme. In particular, between each refresh, the reduction needs to interleave the executions of $\mathrm{T}^{0}$ and $\mathrm{T}^{1}$ until their outputs diverge. Let $i^{*}$ be the number of tampering queries that the simulator performs until triggering a decoding error. The leakage that the reduction needs to perform during this stage (namely, between two consecutive refreshes) is $\mathrm{T}^{0}\left(C_{0}, f_{0}^{0}\right), \mathrm{T}^{1}\left(C_{1}, f_{1}^{0}\right), \ldots, \mathrm{T}^{0}\left(C_{0}, f_{0}^{i^{*}}\right), \mathrm{T}^{1}\left(C_{1}, f_{1}^{i^{*}}\right)$ where $\left(f_{0}^{0}, f_{1}^{0}\right), \ldots,\left(f_{0}^{i^{*}}, f_{1}^{i^{*}}\right)$ is the list of tampering functions chosen by the adversary. We have:

$$
\begin{aligned}
& \widetilde{\mathbb{H}}_{\infty}\left(C_{0} \mid \mathrm{T}^{0}\left(C_{0}, f_{0}^{0}\right), \ldots, \mathrm{T}^{0}\left(C_{0}, f_{0}^{i^{*}}\right)\right)= \\
& \widetilde{\mathbb{H}}_{\infty}\left(C_{0} \mid \mathrm{T}^{1}\left(C_{1}, f_{1}^{0}\right), \ldots, \mathrm{T}^{1}\left(C_{1}, f_{1}^{i^{*}-1}\right), \mathrm{T}^{0}\left(C_{0}, f_{0}^{i^{*}}\right)\right) .
\end{aligned}
$$

In fact, the output of $\mathrm{T}^{0}\left(C_{0}, f_{0}^{i}\right)$ and $\mathrm{T}^{0}\left(C_{0}, f_{0}^{i}\right)$ is, by definition, exactly the same whenever $i<i^{*}$. Moreover, since the output of a function cannot be more informative than its own inputs,

$$
\begin{aligned}
& \widetilde{\mathbb{H}}_{\infty}\left(C_{0} \mid \mathrm{T}^{1}\left(C_{1}, f_{1}^{0}\right), \ldots, \mathrm{T}^{1}\left(C_{1}, f_{1}^{i^{*}-1}\right), \mathrm{T}^{0}\left(C_{0}, f_{0}^{i^{*}}\right)\right) \geq \\
& \widetilde{\mathbb{H}}_{\infty}\left(C_{0} \mid C_{1}, i^{*}, \mathrm{~T}^{0}\left(C_{0}, f_{0}^{i^{*}}\right)\right) .
\end{aligned}
$$


Lastly, we will use the fact that $C_{1}$ reveals only little information about $C_{0}$ (a requirement met by known schemes), and further that $i^{*}$ and $\mathrm{T}^{0}\left(C_{0}, f_{0}^{i^{*}}\right)$ can decrease the min-entropy of $C_{0}$ of at most their size, which is $O(\kappa)$. The reduction, therefore, defines a valid adversary in the CLRS storage-friendly security experiment of the PKE.

Remark 1 (On the refreshing procedure). The feature of split-state refresh does not require that a refreshed codeword be indistinguishable from a freshly sampled one. And, indeed, this is not the case in our construction, as the public key pk (resp. the commitment $\gamma$ ) do not change after the refreshing algorithms are executed. However, the latter property is not required for our proof, as the only thing that matters is that the information about the target codeword that an adversary gathers before a refresh takes place will not be useful after the refresh. Put differently, the adversary could potentially leak the entire values $p k$ and $\gamma$, but this information would not be useful for breaking the security of our scheme.

\subsection{Description of the Simulator}

Let $\mathrm{S}^{0}:=\left(\mathrm{S}_{0}^{0}, \mathrm{~S}_{1}^{0}\right)$ and $\mathrm{S}^{1}:=\left(\mathrm{S}_{0}^{1}, \mathrm{~S}_{1}^{1}\right)$ be the zero-knowledge simulators for the NIAs $\mathcal{N} \mathcal{I} \mathcal{A}_{0}$ and $\mathcal{N} \mathcal{I} \mathcal{A}_{1}$, respectively. We start by defining the simulator $\mathrm{S}_{0}$, whose goal is to simulate the CRS generation for the encoding scheme. Briefly, $\mathrm{S}_{0}$ samples the keys for the PKE scheme and the commitment scheme, and generates an encoding of a dummy message by using simulated proofs in place of real proofs.

Simulator $\mathrm{S}_{0}\left(1^{\kappa}\right)$ :

- For $\beta \in\{0,1\}$ sample $\left(\omega_{\beta}, \tau_{\text {sim }}^{\beta}, \tau_{\text {ext }}^{\beta}\right) \leftarrow \mathrm{S}_{0}^{\beta}\left(1^{\kappa}\right)$.

- Pick $\omega \leftarrow \operatorname{CRSGen}\left(1^{\kappa}\right)$ and $\rho \leftarrow \operatorname{Setup}\left(1^{\kappa}\right)$.

- Define $\tau^{*}:=\left(\tau_{\text {sim }}^{0}, \tau_{\text {ext }}^{0}, \tau_{\text {sim }}^{1}, \tau_{\text {ext }}^{1}\right)$.

- Sample $r \leftarrow\{0,1\}^{*}$, let $\gamma:=\operatorname{Commit}\left(\omega, 0^{\mu} ; r\right)$, and compute $c \leftarrow \operatorname{Enc}\left(p k, 0^{\mu} \| r\right)$, where $\mu \in \mathbb{N}$ is the bit-length associated to the message space $\mathcal{M}$ for the commitment scheme.

- Let $C:=\left(\left(p k, c, \pi_{0}\right),\left(\gamma, s k, \pi_{1}\right)\right):=\left(C_{0}, C_{1}\right)$, where $\pi_{0} \leftarrow \mathrm{S}_{1}^{0}\left(\tau_{\text {sim }}^{0}, c, p k\right)$ and $\pi_{1} \leftarrow \mathrm{S}_{1}^{1}\left(\tau_{\text {sim }}^{1}\right.$, $s k,(\gamma, \omega))$.

- Output $\bar{\omega}:=\left(\omega_{0}, \omega_{1}, \omega, \rho\right)$ and auxiliary information $\alpha:=\left(\tau^{*},\left(C_{0}, C_{1}\right)\right)$.

We also define a simulated refresh algorithm, which will be used in the simulation in place of the original refresh mechanism.

$\underline{\text { Algorithm Rfrsh* }\left(\left(\tau_{\text {sim }}^{0}, \tau_{\text {sim }}^{1}\right),\left(\beta, C_{\beta}\right)\right) \text { : }}$

- For $\beta=0$, parse $C_{0}:=\left(c, p k, \pi_{0}\right)$, compute $c^{\prime} \leftarrow \operatorname{UpdateC}(c)$ and $\pi_{0}^{\prime} \leftarrow \mathrm{S}_{1}^{0}\left(\tau_{\text {sim }}^{0}, c^{\prime}, p k\right)$, and return $C_{0}^{\prime}:=\left(p k, c^{\prime}, \pi_{0}^{\prime}\right)$.

- For $\beta=1$, parse $C_{1}:=\left(\gamma, s k, \pi_{1}\right)$, compute $s k^{\prime} \leftarrow \operatorname{UpdateS}(s k)$, and $\pi_{1}^{\prime} \leftarrow \mathrm{S}_{1}^{1}\left(\tau_{\text {sim }}^{1}, s k^{\prime}\right.$, $(\gamma, \omega))$, and return $C_{1}^{\prime}:=\left(\gamma, s k^{\prime}, \pi_{1}^{\prime}\right)$. 


\section{The Tampering Simulators}

Algorithm $\mathrm{T}^{0}\left(C_{0}, f_{0} ; r_{\text {ext }}^{0}\right)$ :

1. Parse $C_{0}:=\left(p k, c, \pi_{0}\right)$.

2. Compute $\widetilde{C}_{0}=f_{0}\left(C_{0}\right):=\left(\widetilde{p k}, \widetilde{c}, \widetilde{\pi}_{0}\right)$. Hence:

(a) If $\operatorname{Vrfy}_{0}^{\widetilde{c}}\left(\omega_{0}, \widetilde{p k}, \widetilde{\pi}_{0}\right)=0$, set $\widetilde{M}:=\perp$.

(b) If $\left(\widetilde{p k}, \widetilde{c}, \widetilde{\pi}_{0}\right)=\left(p k, c, \pi_{0}\right)$, set $\widetilde{M}=\diamond$.

(c) Else, $\operatorname{run}\left(\widehat{s k}, \phi_{0}, \widehat{c}\right):=\mathrm{K}_{0}\left(\tau_{\text {ext }}^{0}, \widetilde{c}, \widetilde{p k}, \widetilde{\pi}_{0} ; r_{\text {ext }}^{0}\right)$.

(d) Output Abort in case either of the following happens:

i. $\widehat{s k} \neq \perp$ and $\widetilde{p k} \neq \mathrm{PK}(\widehat{s k})$; or

ii. $\left(\phi_{0}, \widehat{c}\right) \neq(\perp, \perp)$ and $\left(\phi_{0}(\widehat{c}) \neq \widetilde{c}\right) \vee\left(\phi_{0} \notin \Phi_{0}\right)$; or

iii. $\left(\widehat{s k}, \phi_{0}, \widehat{c}\right)=(\perp, \perp, \perp)$.

(e) If $\widehat{s k} \neq \perp$ and $\widetilde{p k}=\operatorname{PK}(\widehat{s k})$, let $\widetilde{M} \| \widetilde{r}:=\operatorname{Dec}(\widehat{s k}, \widetilde{c})$.

(f) If $\left(\phi_{0}, \widehat{c}\right) \neq(\perp, \perp)$ and $\left(\phi_{0}(\widehat{c})=\widetilde{c}\right) \wedge\left(\phi_{0} \in \Phi_{0}\right)$, and $\widehat{c}=c$, let $\widetilde{M}:=\diamond$.

3. Return $\widetilde{M}$.

Algorithm $\mathrm{T}^{1}\left(C_{1}, f_{1} ; r_{\text {ext }}^{1}\right)$ :

1. Parse $C_{1}:=\left(\gamma, s k, \pi_{1}\right)$.

2. Compute $\widetilde{C}_{1}=f_{1}\left(C_{1}\right):=\left(\widetilde{\gamma}, \widetilde{s k}, \widetilde{\pi}_{1}\right)$. Hence:

(a) If $\operatorname{Vrfy}_{1}^{\widetilde{s k}}\left(\omega_{1},(\widetilde{\gamma}, \omega), \widetilde{\pi}_{1}\right)=0$, set $\widetilde{M}:=\perp$.

(b) If $\left(\widetilde{\gamma}, \widetilde{s k}, \widetilde{\pi}_{1}\right)=\left(\gamma, s k, \pi_{1}\right)$, set $\widetilde{M}=\diamond$.

(c) Else, run $\left(\widehat{M} \| \widehat{r}, \phi_{1}, \widehat{s k}\right):=\mathrm{K}_{1}\left(\tau_{\text {ext }}^{1}, \widetilde{s k},(\widetilde{\gamma}, \omega), \widetilde{\pi}_{1} ; r_{\text {ext }}^{1}\right)$.

(d) Output Abort in case either of the following happens:

i. $\widehat{M} \| \widehat{r} \neq \perp$ and $\widetilde{\gamma} \neq \operatorname{Commit}(\omega, \widehat{M} ; \widehat{r})$; or

ii. $\left(\phi_{1}, \widehat{s k}\right) \neq(\perp, \perp)$ and $\left(\phi_{1}(\widehat{s k}) \neq \widetilde{s k}\right) \vee\left(\phi_{1} \notin \Phi_{1}\right)$; or

iii. $\left(\widehat{M} \| \widehat{r}, \phi_{1}, \widehat{s k}\right)=(\perp, \perp, \perp)$.

(e) If $\widehat{M} \| \widehat{r} \neq \perp$ and $\widetilde{\gamma}=\operatorname{Commit}(\omega, \widehat{M} ; \widehat{r})$, let $\widetilde{M}:=\widehat{M}$.

(f) If $\left(\phi_{1}, \widehat{s k}\right) \neq(\perp, \perp)$ and $\left(\phi_{1}(\widehat{s k})=\widetilde{s k}\right) \wedge\left(\phi_{1} \in \Phi_{1}\right)$, and $\widehat{s k}=s k$, let $\widetilde{M}:=\diamond$.

3. Return $\widetilde{M}$.

Figure 6: The tampering simulators $\mathrm{T}^{0}$ and $\mathrm{T}^{1}$.

The tampering simulators. We now turn to defining the simulator $S_{1}$. To facilitate the description, in Fig. 6, we formalize two algorithms that we call the tampering simulators. Intuitively, for $\beta \in\{0,1\}$, algorithm $\mathrm{T}^{\beta}$ simulates the outcome corresponding to a tampering query $\left(f_{0}, f_{1}\right)$ from the adversary, by using only $C_{\beta}$ (i.e., one piece of the simulated codeword). On a very high level, as long as both $\mathrm{T}^{0}$ and $\mathrm{T}^{1}$ return the same message $\widetilde{M}$, the simulator $\mathrm{S}_{1}$ uses $\widetilde{M}$ to answer the tampering queries from the adversary; otherwise, in case $\mathrm{T}^{0}$ and $\mathrm{T}^{1}$ disagree, 
it emulates a decoding error.

Simulator $\mathrm{S}_{1}$ :

1. Parse $\alpha:=\left(\left(\tau_{\text {sim }}^{0}, \tau_{\text {ext }}^{0}, \tau_{\text {sim }}^{1}, \tau_{\text {ext }}^{1}\right),\left(C_{0}, C_{1}\right)\right):=\left(\tau^{*}, C\right)$, and run $\operatorname{Rfrsh}^{*}\left(\left(\tau_{\text {sim }}^{0}, \tau_{\text {sim }}^{1}\right), C\right)$. Set the flag err $\leftarrow 0$.

2. Hence, upon a command from the adversary, behave as follows.

(a) Upon input a leakage query (Leak, $\left.\beta, g_{\beta}\right)$, for $\beta \in\{0,1\}$, return $y:=g_{\beta}\left(C_{\beta}\right)$.

(b) Upon input a tampering query $\left(\operatorname{Tamp},\left(f_{0}, f_{1}\right)\right)$, if err $=1$ return $\perp$. Otherwise, let $\widetilde{M}_{0} \leftarrow \mathrm{T}^{0}\left(C_{0}, f_{0}\right)$ and $\widetilde{M}_{1}:=\mathrm{T}^{1}\left(C_{1}, f_{1}\right)$. If either of the tampering simulators returned Abort, abort the simulation outputting the special value Abort. If either $\widetilde{M}_{0}=\perp$, or $\widetilde{M}_{1}=\perp$, return $\perp$ and set err $\leftarrow 1$; else, return $\widetilde{M}:=\widetilde{M}_{0}=\widetilde{M}_{1}$.

(c) Upon input the extra tampering query (Final, $\left.\left(f_{0}^{*}, f_{1}^{*}\right)\right)$, let $\widetilde{M}_{0}^{*}:=\mathrm{T}^{0}\left(C_{0}, f_{0}^{*} ; r_{\text {ext }}^{0}\right)$ and $\widetilde{M}_{1}^{*}:=\mathrm{T}^{1}\left(C_{1}, f_{1}^{*} ; r_{\text {ext }}^{1}\right)$, for random $r_{\text {ext }}^{0}, r_{\text {ext }}^{1} \leftarrow\{0,1\}^{*}$. If either of the tampering simulators returned Abort, abort the simulation outputting the special value Abort. If either $\widetilde{M}_{0}^{*} \neq \widetilde{M}_{1}^{*}$, or $\widetilde{M}_{0}^{*}=\perp$, or $\widetilde{M}_{1}^{*}=\perp$, return $\perp$. Otherwise, if $\widetilde{M}_{0}^{*}=\widetilde{M}_{1}^{*}=\diamond$, return $\diamond$. Else, proceed as follows:

i. Let $\widetilde{C}_{0}^{*}:=f_{0}^{*}\left(C_{0}\right):=\left(\widetilde{p k}, \widetilde{c}, \widetilde{\pi}_{0}\right)$ and $\widetilde{C}_{1}^{*}:=f_{1}^{*}\left(C_{1}\right):=\left(\widetilde{\gamma}, \widetilde{s k}, \widetilde{\pi}_{1}\right)$.

ii. Extract the values $\left(\widehat{s k}_{0}, \phi_{0}, \widehat{c}_{0}\right):=\mathrm{K}_{0}\left(\tau_{\text {ext }}^{0}, \widetilde{c}, \widetilde{p k}, \widetilde{\pi}_{0} ; r_{\text {ext }}^{0}\right)$, and let $s k^{\prime}=\operatorname{UpdateS}\left(\widehat{s k}_{0}\right)$.

iii. Extract the values $\left((\widehat{M}, \widehat{r}), \phi_{1}, \widehat{s k}_{1}\right):=\mathrm{K}_{1}\left(\tau_{\text {ext }}^{1}, \widetilde{s k},(\widetilde{\gamma}, \omega), \widetilde{\pi}_{1} ; r_{\text {ext }}^{1}\right)$, and compute $c^{\prime} \leftarrow \operatorname{Enc}(\widetilde{p k}, \widehat{M} \| \widehat{r}) \cdot{ }^{9}$

iv. Run $\widetilde{\pi}_{0}^{\prime} \leftarrow \mathrm{S}_{1}^{0}\left(\tau_{\text {sim }}^{0}, c^{\prime}, \widetilde{p k}\right)$ and $\widetilde{\pi}_{1}^{\prime} \leftarrow \mathrm{S}_{1}^{1}\left(\tau_{\text {sim }}^{1}, s k^{\prime},(\widetilde{\gamma}, \omega)\right)$.

v. Output $\widetilde{C}^{\prime}:=\left(\left(\widetilde{p k}, c^{\prime}, \widetilde{\pi}_{0}^{\prime}\right),\left(\widetilde{\gamma}, s k^{\prime}, \widetilde{\pi}_{1}^{\prime}\right)\right)$.

3. Go back to step 1 until the adversary is done with its queries.

\subsection{Hybrids}

We define a sequence of games, starting with the simulated experiment $\operatorname{SimTamper}_{\mathrm{A}, \mathrm{S}}(\kappa, \ell, q)$ for the above defined simulator $S=\left(S_{0}, S_{1}\right)$. The games are described in an incremental manner, meaning that for each game we only highlight the differences with the previous game, implicitly assuming that all other steps are unchanged. Each game is parameterized by the adversary A and, implicitly, by a simulator that is typically derived by the original simulator $\mathrm{S}$.

Game $\mathbf{G}_{\mathrm{A}}^{0}(\kappa)$. This game is identical to $\operatorname{SimTamper}_{\mathrm{A}, \mathrm{S}}(\kappa, \ell, q)$, for the above defined simulator $\mathrm{S}=\left(\mathrm{S}_{0}, \mathrm{~S}_{1}\right)$.

Game $\mathbf{G}_{\mathrm{A}}^{1}(\kappa)$. The simulator $\mathrm{S}_{0}$ is additionally given $M$ as input, and computes the commitment in the simulated codeword $\left(C_{0}, C_{1}\right)$ as $\gamma=\operatorname{Commit}(\omega, M ; r)$, for $r \leftarrow\{0,1\}^{*}$.

Game $\mathbf{G}_{\mathrm{A}}^{2}(\kappa)$. The simulator $\mathrm{S}_{0}$ computes the ciphertext in the simulated codeword $\left(C_{0}, C_{1}\right)$ as $c \leftarrow \operatorname{Enc}(p k, M \| r)$.

Game $\mathbf{G}_{\mathrm{A}}^{3}(\kappa)$. The simulator $\mathrm{S}_{1}$ keeps track of all the ciphertexts and secret keys contained in each dummy encoding computed in step 1. Namely, for each $i \in[q]$, it lets $\alpha \leftarrow \alpha \|\left(c_{i}, s k_{i}\right)$, where $c_{i}, s k_{i}$ are, respectively, the ciphertext and the secret key as obtained by refreshing the initial encoding $C$ using procedure Rfrsh*.

\footnotetext{
${ }^{9}$ Note that the extractors $\mathrm{K}_{0}$ and $\mathrm{K}_{1}$ are run, respectively, using the same random coins on which the tampering simulators $\mathrm{T}^{0}$ and $\mathrm{T}^{1}$ are run.
} 
Game $\mathbf{G}_{\mathrm{A}}^{4}(\kappa)$. For each round $i \in[q]$, the tampering simulators $\mathrm{T}^{0}$ and $\mathrm{T}^{1}$ are modified as follows (cf. Fig. 6):

$\mathrm{T}^{0}=\left(C_{0}, f_{0}\right):$

- The check at step $2(\mathrm{~d})$ ii becomes $\left(\phi_{0}, \widehat{c}\right) \neq(\perp, \perp)$ and $\left(\phi_{0}(\widehat{c}) \neq \widetilde{c}\right) \vee\left(\phi_{0} \notin \Phi_{0}\right) \vee(\widehat{c} \notin$ $\left.\left\{c_{1}, \ldots, c_{i-1}\right\}\right)$.

- The simulator additionally sets $\widetilde{M}=\diamond$ at step $2 \mathrm{~b}$ if $\widetilde{c} \in\left\{c_{1}, \ldots, c_{i-1}\right\}$.

$\mathrm{T}^{1}=\left(C_{1}, f_{1}\right):$

- The check at step $2(\mathrm{~d})$ ii becomes $\left(\phi_{1}, \widehat{s k}\right) \neq(\perp, \perp)$ and $\left(\phi_{1}(\widehat{s k}) \neq \widetilde{s k}\right) \vee\left(\phi_{1} \notin\right.$ $\left.\Phi_{1}\right) \vee\left(\widehat{s k} \notin\left\{s k_{1}, \ldots, s k_{i-1}\right\}\right)$.

- The simulator additionally sets $\widetilde{M}=\diamond$ at step 2 b if $\widetilde{s k} \in\left\{s k_{1}, \ldots, s k_{i-1}\right\}$.

Game $\mathbf{G}_{\mathbf{A}}^{5}(\kappa)$. We change the way tampering queries for which the simulator returns $\diamond$ are treated. In particular,

- For each query (Tamp, $\left(f_{0}, f_{1}\right)$ ) such that $\mathrm{S}_{1}$ outputs $\widetilde{M}=\diamond$, we treat this query exactly as in the real experiment (i.e., we apply $\left(f_{0}, f_{1}\right)$ to the target encoding and decode the resulting codeword).

- For each query (Final, $\left.\left(f_{0}^{*}, f_{1}^{*}\right)\right)$ such that $\mathrm{S}_{1}$ outputs $\diamond$, we treat this query exactly as in the real experiment (i.e., we apply $\left(f_{0}^{*}, f_{1}^{*}\right)$ to the target encoding and decode the resulting codeword).

Game $\mathbf{G}_{A}^{6}(\kappa)$. We change the way tampering queries for which the simulator returns a value which is not in $\{\perp, \diamond\}$ are treated. In particular,

- For each query (Tamp, $\left.\left(f_{0}, f_{1}\right)\right)$ such that $\mathrm{S}_{1}$ outputs $\widetilde{M}=\widetilde{M}_{0}=\widetilde{M}_{1}$, we treat this query exactly as in the real experiment (i.e., we apply $\left(f_{0}, f_{1}\right)$ to the target encoding and decode the resulting codeword).

- For each query (Final, $\left.\left(f_{0}^{*}, f_{1}^{*}\right)\right)$ such that $S_{1}$ outputs a codeword $\widetilde{C}^{\prime}$ that is valid but does not decode to $M$, we treat this query exactly as in the real experiment (i.e., we apply $\left(f_{0}^{*}, f_{1}^{*}\right)$ to the target encoding and decode the resulting codeword).

Game $\mathbf{G}_{A}^{7}(\kappa)$. This game is identical to the previous one, except that the tampering simulators $\mathrm{T}^{0}$ and $\mathrm{T}^{1}$ do not perform the check in step 2 d, i.e. in game $\mathbf{G}_{\mathrm{A}}^{7}(\kappa)$ the tampering simulators never output Abort (and so does $\mathrm{S}_{1}$ ).

Game $\mathbf{G}_{A}^{8}(\kappa)$. We change the way tampering queries for which the simulator returns $\perp$ are treated. In particular,

- For each query (Tamp, $\left.\left(f_{0}, f_{1}\right)\right)$ such that $\mathrm{S}_{1}$ outputs $\widetilde{M}=\perp$, we treat this query exactly as in the real experiment (i.e., we apply $\left(f_{0}, f_{1}\right)$ to the target encoding and decode the resulting codeword).

- For each query (Final, $\left.\left(f_{0}^{*}, f_{1}^{*}\right)\right)$ such that $S_{1}$ outputs $\perp$, we treat this query exactly as in the real experiment (i.e., we apply $\left(f_{0}^{*}, f_{1}^{*}\right)$ to the target encoding and decode the resulting codeword).

Game $\mathbf{G}_{\mathrm{A}}^{9}(\kappa)$. We change the way the codeword $\widetilde{C}_{0}^{*}$ corresponding to the extra tampering query is computed in each round. Namely, instead of running the extractor $\mathrm{K}_{0}$ and letting $s k^{\prime} \leftarrow$ UpdateS $(\widehat{s k})$ (where $\widehat{s k}$ is the extracted secret key), we directly let $s k^{\prime} \leftarrow \operatorname{UpdateS}(\widetilde{s k})$. 
Game $\mathbf{G}_{\mathrm{A}}^{10}(\kappa)$. We change the way the codeword $\widetilde{C}_{1}^{*}$ corresponding to the extra tampering query is computed in each round. Namely, instead of running the extractor $\mathrm{K}_{1}$ and letting $c^{\prime} \leftarrow \operatorname{Enc}(p k, \widehat{M} \| \widehat{r})$ (where $\widehat{M} \| \widehat{r}$ is the extracted message/randomness), we directly let $c^{\prime} \leftarrow \operatorname{UpdateC}(\widetilde{c})$.

Game $\mathbf{G}_{\mathrm{A}}^{11}(\kappa)$. We setup the CRS differently. Namely, we change the distribution of $\omega_{0}$ as in $\omega_{0} \leftarrow \mathrm{CRSGen}_{0}\left(1^{\kappa}\right)$. Moreover, the simulator $\mathrm{S}_{1}$ always runs the prover algorithm Prove $_{0}$ instead of the zero-knowledge simulator $S_{1}^{0}$. (Observe that this happens in three different steps: (i) When the original codeword $\left(C_{0}, C_{1}\right)$ is computed; (ii) Whenever $\mathrm{S}_{1}$ runs algorithm Rfrsh* at the beginning of each round; (iii) Whenever $S_{1}$ needs to answer the extra tampering query.)

Game $\mathbf{G}_{\mathrm{A}}^{12}(\kappa)$. We setup the CRS differently. Namely, we change the distribution of $\omega_{1}$ as in $\omega_{1} \leftarrow \operatorname{CRSGen}_{1}\left(1^{\kappa}\right)$. Moreover, the simulator $S_{1}$ always runs the prover algorithm Prove ${ }_{1}$ instead of the zero-knowledge simulator $S_{1}^{1}$. (Once again, this happens in the exact same cases as in the previous game.)

Game $\mathbf{G}_{A}^{13}(\kappa)$. We change the way a codeword is refreshed. Namely, instead of computing $\pi_{0}^{\prime} \leftarrow \operatorname{Prove}_{0}^{c^{\prime}}\left(\omega_{0}, p k, s k\right)$ via the prover algorithm, we now run the label evaluation procedure as done by the real refresh algorithm. The same modification affects the way the proof $\pi_{0}^{\prime}$ is computed during each of the extra tampering queries $\left(f_{0}^{*}, f_{1}^{*}\right)$.

Game $\mathbf{G}_{\mathrm{A}}^{14}(\kappa)$. We change the way a codeword is refreshed. Namely, instead of computing $\pi_{1}^{\prime} \leftarrow \operatorname{Prove}_{1}^{s k^{\prime}}\left(\omega_{1},(\omega, \gamma),(M, r)\right)$ via the prover algorithm, we now run the label evaluation procedure as done by the real refresh algorithm. The same modification affects the way the proof $\pi_{1}^{\prime}$ is computed during each of the extra tampering queries $\left(f_{0}^{*}, f_{1}^{*}\right)$.

Notice that in game $\mathbf{G}_{\mathrm{A}}^{14}(\kappa)$, the "simulator" $\mathrm{S}_{1}$ computes an encoding of the message $M$. Moreover, tampering queries are always answered by decoding the modified codeword, and codewords are refreshed exactly as in the real experiment. Thus, $\mathbf{G}_{\mathbf{A}}^{14}(\kappa)$ and $\operatorname{Tamper}_{\mathcal{C S}, \mathbf{A}}(\kappa, \ell, q)$ are identically distributed.

\subsection{Indistinguishability of the Hybrids}

We now prove that the above defined games are computationally indistinguishable. Together with the fact that the last game is identically distributed to the real tampering experiment, this implies Theorem 1.

Lemma 3. For all PPT adversaries $\mathrm{A}$ there exists a negligible function $\nu_{0,1}: \mathbb{N} \rightarrow[0,1]$ such that $\left|\mathbb{P}\left[\mathbf{G}_{\mathbf{A}}^{0}(\kappa)=1\right]-\mathbb{P}\left[\mathbf{G}_{\mathbf{A}}^{1}(\kappa)=1\right]\right| \leq \nu_{0,1}(\kappa)$.

Proof sketch. The proof is down to the hiding property of the commitment scheme (cf. Definition 8). Since the reduction is straightforward, we only highlight the main ideas here and leave the details to the reader.

Assume there exists a PPT adversary $\mathrm{A}$ and a polynomial $p_{0,1}(\cdot)$ such that, for infinitely many values of $\kappa \in \mathbb{N}$, we have $\left|\mathbb{P}\left[\mathbf{G}_{\mathrm{A}}^{0}(\kappa)=1\right]-\mathbb{P}\left[\mathbf{G}_{\mathrm{A}}^{1}(\kappa)=1\right]\right| \geq 1 / p_{0,1}(\kappa)$. We construct a PPT adversary $\mathbf{B}$ (with black-box access to $A$ ), that is playing either $\mathbf{G}_{\mathcal{C} \mathcal{O} \mathcal{M}, \mathrm{B}}^{\text {hide }}(\kappa, 0)$ or $\mathbf{G}_{\mathcal{C O M}, \mathrm{B}}^{\text {hide }}(\kappa, 1)$. Roughly, B works as follows:

- Receive $\omega$ from the challenger. Compute $\bar{\omega}$ as $S_{0}\left(1^{\kappa}\right)$ would do it, except that the CRS for the commitment scheme is set to be equal to the CRS $\omega$ from the game. 
- Run $\mathrm{A}_{0}(\bar{\omega})$, obtaining a message $M$. Forward $\left(0^{\mu}, M\right)$ to the challenger, receiving back a commitment $\gamma$.

- Sample the simulated codeword $\left(C_{0}, C_{1}\right)$ exactly as $\mathrm{S}_{0}$ would do it, except that the commitment is set to be equal to $\gamma$ from the game.

- Continue running $A$ and answer its queries exactly as $S_{1}$ would do it.

- Return the same output as that of A.

It is immediate to see that if $\mathrm{B}$ is playing $\mathbf{G}_{\mathcal{C O} \mathcal{M}, \mathrm{B}}^{\text {hide }}(\kappa, 0)$, then the view of $\mathrm{A}$ when running as a sub-routine of $B$ is identical to the view in game $\mathbf{G}_{\mathrm{A}}^{0}(\kappa)$; similarly, if $\mathrm{B}$ is playing $\mathbf{G}_{\mathcal{C} \mathcal{O} \mathcal{M}, \mathrm{B}}^{\text {hide }}(\kappa, 1)$, then the view of $A$ when running as a sub-routine of $B$ is identical to the view in game $\mathbf{G}_{A}^{1}(\kappa)$. Hence, $B$ retains the same advantage of $A$, concluding the proof of the lemma.

Lemma 4. For all PPT adversaries $\mathrm{A}$ there exists a negligible function $\nu_{1,2}: \mathbb{N} \rightarrow[0,1]$ such that $\left|\mathbb{P}\left[\mathbf{G}_{\mathrm{A}}^{1}(\kappa)=1\right]-\mathbb{P}\left[\mathbf{G}_{\mathrm{A}}^{2}(\kappa)=1\right]\right| \leq \nu_{1,2}(\kappa)$.

Proof. The proof is down to the fact that $\mathcal{P K \mathcal { E }}$ is an $\ell$-noisy CLRS friendly PKE scheme. By contradiction, assume there exists a PPT adversary $\mathrm{A}$ and a polynomial $p_{1,2}(\cdot)$ such that, for infinitely many values of $\kappa \in \mathbb{N}$, we have $\left|\mathbb{P}\left[\mathbf{G}_{\mathbf{A}}^{1}(\kappa)=1\right]-\mathbb{P}\left[\mathbf{G}_{\mathbf{A}}^{2}(\kappa)=1\right]\right| \geq 1 / p_{1,2}(\kappa)$. We construct a PPT adversary $\mathrm{B}$ (with black-box access to $\mathrm{A}$ ), that is playing game $\mathbf{G}_{\mathcal{P} \mathcal{K} \mathcal{E}, \mathrm{B}}^{\text {clrs }}\left(\kappa, \ell^{\prime \prime}\right)$, with $\ell^{\prime \prime}:=\ell^{\prime}-2 \kappa$, and $\ell^{\prime}$ as in the statement of Theorem 1. A formal description of B follows.

Adversary B:

1. Receive $(\rho, p k)$ from the challenger. For all $\beta \in\{0,1\}$, sample $\left(\omega_{\beta}, \tau_{\text {sim }}^{\beta}, \tau_{\text {ext }}^{\beta}\right) \leftarrow \mathrm{S}_{0}^{\beta}\left(1^{\kappa}\right)$, and $\omega \leftarrow \operatorname{CRSGen}\left(1^{\kappa}\right)$. Let $\bar{\omega}=\left(\omega_{0}, \omega_{1}, \omega, \rho\right)$ and $\tau^{*}=\left(\tau_{\text {sim }}^{0}, \tau_{\text {ext }}^{0}, \tau_{\text {sim }}^{1}, \tau_{\text {ext }}^{1}\right)$.

2. Run $\mathrm{A}_{0}(\bar{\omega})$, obtaining a message $M \in \mathcal{M}$. Forward $\left(0^{\mu}\|r, M\| r\right)$ to the challenger, for $r \leftarrow\{0,1\}^{*}$, and let $\gamma:=\operatorname{Commit}(\omega, M ; r)$.

3. Repeat the following, until $\mathrm{A}$ is done with its queries.

(a) Query Update(0) and Update(1), sample uniform coins $r^{0}, r^{1} \leftarrow\{0,1\}^{*}$, and set the flag err $\leftarrow 0$.

(b) Upon input (Leak, $\left.\beta, g_{\beta}\right)$, forward $\left(\beta, g_{\beta}\right)$ to the target leakage oracle, receiving back a value $y$; send $y$ to $A$.

(c) Upon input (Tamp, $\left(f_{0}, f_{1}\right)$ ), answer this query exactly as $S_{1}$ would do, except that the values $\widetilde{M}_{0}$ and $\widetilde{M}_{1}$ are obtained, respectively, via queries $\left(0, g_{0}\right)$ and $\left(1, g_{1}\right)$ to the target leakage oracle, where the functions $g_{0}, g_{1}$ are defined as follows:

$$
\begin{aligned}
& g_{0}(\cdot):=\mathrm{T}^{0}\left(\left(p k, \cdot, \mathrm{S}_{1}^{0}\left(\tau_{\text {sim }}^{0}, \cdot, p k ; r^{0}\right), f_{0}\right)\right. \\
& g_{1}(\cdot):=\mathrm{T}^{1}\left(\left(\gamma, \cdot, \mathrm{S}_{1}^{1}\left(\tau_{\text {sim }}^{1}, \cdot,(\gamma, \omega) ; r^{1}\right), f_{1}\right) .\right.
\end{aligned}
$$

(Notice that the above might eventually lead to set the flag err $\leftarrow 1$.)

(d) Upon input (Final, $\left(f_{0}^{*}, f_{1}^{*}\right)$ ), answer this query exactly as $\mathrm{S}_{1}$ would do, except for the following differences.

i. The values $\widetilde{M}_{0}^{*}, \widetilde{M}_{1}^{*}$ are obtained via leakage queries $\left(0, g_{0}\right),\left(1, g_{1}\right)$, where the functions $g_{0}, g_{1}$ are defined as in Eq. (1) and Eq. (2) but with $f_{0}$ replaced by $f_{0}^{*}$ and $f_{1}$ replaced by $f_{1}^{*}$. 
ii. The values $\widetilde{p k}, s k^{\prime}$ (as part of the simulated codeword $\widetilde{C}^{\prime}$ ) are obtained via a leakage query $\left(0, g_{0}^{*}\right)$, where the function $g_{0}^{*}(c)$ hard-wires values $\left(p k, \tau_{\text {sim }}^{0}, r^{0}, \tau_{\text {ext }}^{0}\right.$, $\left.r_{\text {ext }}^{0}\right)$ and behaves as follows: Compute $\widetilde{C}_{0}^{*}:=f_{0}^{*}\left(p k, c, \mathrm{~S}_{1}^{0}\left(\tau_{\text {sim }}^{0}, c, p k ; r^{0}\right)\right):=$ $\left(\widetilde{p k}, \widetilde{c}, \widetilde{\pi}_{0}\right)$, extract the values $\left(\widehat{s k}_{0}, \phi_{0}, \widehat{c}_{0}\right):=\mathrm{K}_{0}\left(\tau_{\text {ext }}^{0}, \widetilde{c}, \widetilde{p k}, \widetilde{\pi}_{0} ; r_{\text {ext }}^{0}\right)$, and output $\left(\widetilde{p k}, s k^{\prime}\right)$, where $s k^{\prime} \leftarrow \operatorname{UpdateS}\left(\widehat{s k}_{0}\right)$.

iii. The values $\widetilde{\gamma}, c^{\prime}$ (as part of the simulated codeword $\widetilde{C}^{\prime}$ ) are obtained via a leakage query $\left(1, g_{1}^{*}\right)$, where the function $g_{\widetilde{C}}^{*}(s k)$ hard-wires values $\left(\omega, \gamma, \tau_{\text {sim }}^{1}, r^{1}, \tau_{\text {ext }}^{1}, r_{\text {ext }}^{1}\right)$ and behaves as follows: Compute $\widetilde{C}_{1}^{*}:=f_{1}^{*}\left(\gamma, s k, \mathrm{~S}_{1}^{1}\left(\tau_{\operatorname{sim}}^{1}, s k,(\omega, \gamma) ; r^{1}\right)\right):=(\widetilde{\gamma}, \widetilde{s k}$, $\left.\widetilde{\pi}_{1}\right)$, extract the values $\left((\widehat{M}, \widehat{r}), \phi_{1}, \widehat{s k}_{1}\right):=\mathrm{K}_{1}\left(\tau_{\text {ext }}^{1}, \widetilde{s k},(\widetilde{\gamma}, \omega), \widetilde{\pi}_{1} ; r_{\text {ext }}^{1}\right)$, and output $\left(\widetilde{\gamma}, c^{\prime}\right)$, where $c^{\prime} \leftarrow \operatorname{Enc}(\widetilde{p k}, \widehat{M} \| \widehat{r})$.

4. Output the same as A does.

Notice that, assuming the leakage bound is not violated, adversary B perfectly simulates the view of $A$; namely, if the challenge bit is $b=0$, the view of $A$ is identically distributed to that of game $\mathbf{G}_{\mathrm{A}}^{1}$, and if the challenge bit is $b=1$, the view of $\mathrm{A}$ is identically distributed to that of game $\mathbf{G}_{\mathrm{A}}^{2}$. Thus,

$$
\begin{aligned}
1 / p_{1,2}(\kappa) & \leq\left|\mathbb{P}\left[\mathbf{G}_{\mathrm{A}}^{1}(\kappa)=1\right]-\mathbb{P}\left[\mathbf{G}_{\mathrm{A}}^{2}(\kappa)=1\right]\right| \\
& =\left|\mathbb{P}\left[\mathbf{G}_{\mathcal{P K} \mathcal{E}, \mathrm{B}}^{\mathrm{clrs}}\left(\kappa, \ell^{\prime \prime}\right)=1 \mid b=1\right]-\mathbb{P}\left[\mathbf{G}_{\mathcal{P K} \mathcal{E}, \mathrm{B}}^{\text {clrs }}\left(\kappa, \ell^{\prime \prime}\right)=0 \mid b=0\right]\right| \\
& =2\left|\mathbb{P}\left[\mathbf{G}_{\mathcal{P} \mathcal{K}, \mathrm{B}}^{\mathrm{clr}}\left(\kappa, \ell^{\prime \prime}\right)=1\right]-\frac{1}{2}\right|
\end{aligned}
$$

contradicting the fact that $\mathcal{P K \mathcal { E }}$ is a CLRS friendly PKE scheme.

It remains to show that the adversary $B$ does not violate the leakage bound as stated in Theorem 1. To this end, assume that, between each update, the adversary $A$ makes at most $t \in \operatorname{poly}(\kappa)$ tampering queries (not counting the final extra query $\left(f_{0}^{*}, f_{1}^{*}\right)$ ), and let $i^{*} \in[t]$ be the first index for which we have $\widetilde{M}_{0, i^{*}}:=\mathrm{T}^{0}\left(C_{0}, f_{0}^{i^{*}}\right) \neq \mathrm{T}^{1}\left(C_{1}, f_{1}^{i^{*}}\right):=\widetilde{M}_{1, i^{*}}$. Define

$$
Z:=\left(Z_{0}, Z_{1}\right):=\left(\left(Y_{0}, \widetilde{M}_{0,1}, \ldots \widetilde{M}_{0, i^{*}}, \widetilde{M}_{0}^{*}\right),\left(Y_{1}, \widetilde{M}_{1,1}, \ldots, \widetilde{M}_{1, i^{*}}, \widetilde{M}_{1}^{*}\right)\right)
$$

to be the random variable corresponding to the partial view of $\mathrm{A}$ in each epoch, up until step $3 \mathrm{c}$ included, which consists of the leakage $Y_{0}$ done on $C_{0}$, the leakage $Y_{1}$ done on $C_{1}$, and the messages $\widetilde{M}_{0,1}, \ldots \widetilde{M}_{0, i^{*}}, \widetilde{M}_{0}^{*}$ and $\widetilde{M}_{1,1}, \ldots \widetilde{M}_{1, i^{*}}, \widetilde{M}_{1}^{*}$ as defined by the tampering simulators $\mathrm{T}^{0}$ and $\mathrm{T}^{1}$. For $\beta \in\{0,1\}$, let $X_{\beta}$ be the random variable corresponding to $C_{\beta}$ conditioned on the value $p k$, and notice that, after each update (performed by the reduction in step 3a), $X_{0}$ and $X_{1}$ are independent. We can write:

$$
\begin{aligned}
\widetilde{\mathbb{H}}_{\infty}\left(X_{\beta} \mid Z_{\beta}\right) & =\widetilde{\mathbb{H}}_{\infty}\left(X_{\beta} \mid Y_{\beta}, \widetilde{M}_{\beta, 1}, \ldots, \widetilde{M}_{\beta, i^{*}-1}, \widetilde{M}_{\beta, i^{*}}, \widetilde{M}_{\beta}^{*}\right) \\
& =\widetilde{\mathbb{H}}_{\infty}\left(X_{\beta} \mid Y_{\beta}, \widetilde{M}_{1-\beta, 1}, \ldots, \widetilde{M}_{1-\beta, i^{*}-1}, \widetilde{M}_{\beta, i^{*}}, \widetilde{M}_{\beta}^{*}\right) \\
& \geq \widetilde{\mathbb{H}}_{\infty}\left(X_{\beta} \mid Y_{\beta}, X_{1-\beta}, i^{*}, \widetilde{M}_{\beta, i^{*}}, \widetilde{M}_{\beta}^{*}\right) \\
& =\widetilde{\mathbb{H}}_{\infty}\left(X_{\beta} \mid Y_{\beta}, i^{*}, \widetilde{M}_{\beta, i^{*}}, \widetilde{M}_{\beta}^{*}\right) \\
& \geq \ell-3 \mu
\end{aligned}
$$

where Eq. (3) uses the definition of the random variable $Z_{\beta}$, Eq. (4) uses the fact that $\widetilde{M}_{0, i}=\widetilde{M}_{1, i}$ for all $i<i^{*}$, Eq. (5) follows by Lemma 1 and the fact that the messages $\widetilde{M}_{1-\beta, 1}, \ldots, \widetilde{M}_{1-\beta, i^{*}-1}$ can be computed as a deterministic function of $X_{1-\beta}$ and the index $i^{*}$, Eq. (6) uses the independence between $X_{0}$ and $X_{1}$, and Eq. (7) follows by Lemma 2, using the fact that $\mathrm{A}$ is $\ell$-valid and by the definition of $\mu:=\log |\mathcal{M}|$. 
It remains to account for the leakage performed by the reduction to answer the final tampering query before the state is updated. In particular:

- In step 3(d)ii, B leaks the public key $\widetilde{p k}$ and the secret key $s k^{\prime}$; since $s k^{\prime}$ is uniformly distributed over the set $\{s k: \operatorname{PK}(s k)=\widetilde{p k}\}$, the function $g_{0}^{*}$ is at most $\log |\mathcal{P K}|=\delta$ leaky.

- In step 3(d)iii, B leaks the commitment $\widetilde{\gamma}$ and the ciphertext $c^{\prime}$; since $c^{\prime}$ is uniformly distributed over the set $\{c: \operatorname{Enc}(\widetilde{p k}, \widehat{M} \| \widehat{r})\}$, the function $g_{1}^{*}$ is at most $\log \left|\mathcal{M}_{\text {pke }}\right|=\mu_{\text {pke}^{-}}$ leaky.

Putting the above facts together with the bound from Eq. 7, we obtain that B is $\ell^{\prime \prime}$-admissible for $\ell^{\prime \prime}:=\ell+3 \mu+\max \left\{\delta, \mu_{\mathrm{pke}}\right\}=\ell^{\prime}-2 \kappa$. This concludes the proof.

Lemma 5. For any $\kappa \in \mathbb{N}$, we have $\mathbf{G}_{\mathbf{A}}^{2}(\kappa) \equiv \mathbf{G}_{\mathbf{A}}^{3}(\kappa)$.

Proof. Follows directly by the fact that the change in the game $\mathbf{G}_{\mathbf{A}}^{3}(\kappa)$ is only syntactical, and in particular the view of the adversary is identical in the two games.

Lemma 6. For all PPT adversaries $\mathrm{A}$ there exists a negligible function $\nu_{3,4}: \mathbb{N} \rightarrow[0,1]$ such that $\left|\mathbb{P}\left[\mathbf{G}_{\mathrm{A}}^{3}(\kappa)=1\right]-\mathbb{P}\left[\mathbf{G}_{\mathrm{A}}^{4}(\kappa)=1\right]\right| \leq \nu_{3,4}(\kappa)$.

Proof. The proof is again down to the security of the CLRS friendly PKE scheme. Consider the following events:

$0 \mathrm{~d}_{0}$ : The event becomes true whenever, for some $i \in[q]$, either the adversary generates a tampered ciphertext $\widetilde{c} \in\left\{c_{1}, \ldots, c_{i-1}\right\}$, or the tampering simulator $\mathrm{T}^{0}$ extracts a ciphertext $\widehat{c} \in\left\{c_{1}, \ldots, c_{i-1}\right\}$.

$0 \mathrm{Od}_{1}$ : The event becomes true whenever, for some $i \in[q]$, either the adversary generates a tampered secret key $\widetilde{s k} \in\left\{s k_{1}, \ldots, s k_{i-1}\right\}$, or the tampering simulator $\mathrm{T}^{1}$ extracts a secret key $\widehat{s k} \in\left\{s k_{1}, \ldots, s k_{i-1}\right\}$.

Define $01 d=0 l d_{0} \vee 0 l d_{1}$. Clearly, $\mathbf{G}_{A}^{3}(\kappa)$ and $\mathbf{G}_{A}^{4}(\kappa)$ are identically distributed conditioned on old not happening. Hence,

$$
\left|\mathbb{P}\left[\mathbf{G}_{\mathrm{A}}^{3}(\kappa)=1\right]-\mathbb{P}\left[\mathbf{G}_{\mathrm{A}}^{4}(\kappa)=1\right]\right| \leq \mathbb{P}[\mathrm{Old}] .
$$

We now show that 0ld only happens with negligible probability. By contradiction, assume there is an efficient adversary $A$ and a polynomial $p_{3,4}$ such that $A$ provokes event $01 d$ with probability at least $1 / p_{3,4}(\kappa)$. We construct an adversary $\mathrm{B}$ (with black-box access to $\mathrm{A}$ ), that is playing game $\mathbf{G}_{\mathcal{P} \mathcal{K} \text {, } \mathrm{B}}^{\text {clrs }}(\kappa, \ell)$, with the parameter $\ell$ as in the statement of Theorem 1 . The execution of $\mathrm{B}$ is almost identical to that of the reduction used to prove Lemma 4; hence, we only outline the main differences between the two reductions below.

- At the beginning B additionally samples $h_{0} \leftarrow \mathcal{H}_{0}$ and $h_{1} \leftarrow \mathcal{H}_{1}$, where $\mathcal{H}_{0}:=\left\{h_{0}\right.$ : $\left.\mathcal{C} \rightarrow\{0,1\}^{\kappa}\right\}$ and $\mathcal{H}_{1}:=\left\{h_{1}: \mathcal{S K} \rightarrow\{0,1\}^{\kappa}\right\}$ are families of pair-wise independent hash functions. ${ }^{10}$

\footnotetext{
${ }^{10}$ Recall that $\mathcal{H}:=\{h: \mathcal{X} \rightarrow \mathcal{Y}\}$ is a family of pair-wise independent hash functions, if the following condition holds: For all distinct $x, x^{\prime} \in \mathcal{X}$, and any $y, y^{\prime} \in \mathcal{Y}$,

$$
\mathbb{P}_{h \leftarrow \mathcal{H}}\left[h(x)=y \wedge h\left(x^{\prime}\right)=y^{\prime}\right] \leq|\mathcal{Y}|^{-2} .
$$
}


- The challenge messages are chosen to be $\left(0^{\mu} \| r, 1^{\mu_{\mathrm{pke}}}\right)$. (The choice of the second message is completely arbitrary, in fact any value in $\mathcal{M}_{\text {pke }}$ would do.)

- Before running the adversary, in each round $i \in[q]$, the reduction makes leakage queries $\left(0, h_{0}\right)$ and $\left(1, h_{1}\right)$, obtaining $y_{i}^{0}=h_{0}\left(c_{i}\right)$ and $y_{i}^{1}=h_{1}\left(s k_{i}\right)$, where $c_{i}$ and $s k_{i}$ are, respectively, the challenge ciphertext and the secret key after the $i$-th update.

- The leakage function $g_{0}$ of Eq. (1), additionally hard-wires a description of $h_{0}$, and all values $y_{1}^{0}, \ldots, y_{i-1}^{0}$, and it checks if either $y_{j}^{0}=h_{0}(\widetilde{c})$ or $y_{j}^{0}=h_{0}(\widehat{c})$, for some $j \leq i-1$; in case that happens, the leakage function returns $\left(0 \mathrm{Od}_{0}, c^{*}\right)$, where $c^{*} \in\{\widetilde{c}, \widehat{c}\}$ is such that $y_{j}^{0}=h_{0}\left(c^{*}\right)$, and otherwise it behaves as before.

- The leakage function $g_{1}$ of Eq. (2), additionally hard-wires a description of $h_{1}$, and all values $y_{1}^{1}, \ldots, y_{i-1}^{1}$, and it checks if either $y_{j}^{1}=h_{1}(\widetilde{s k})$ or $y_{j}^{1}=h_{1}(\widehat{s k})$, for some $j \leq i-1$; in case that happens, the leakage function returns $\left(0 \mathrm{ld}_{1}, s k^{*}\right)$, where $s k^{*} \in\{\widetilde{s k}, \widehat{s k}\}$ is such that $y_{j}^{1}=h_{1}\left(s k^{*}\right)$, and otherwise it behaves as before.

- After the adversary is done with tampering queries, the reduction checks whether for some of the leakage queries it received either a pair $\left(0 \mathrm{ld}_{0}, c^{*}\right)$ or a pair $\left(0 \mathrm{ld}_{1}, s k^{*}\right)$. Thus,

- In the former case, it asks an additional leakage query $\left(1, g_{c^{*}, M \| r, 1^{\mu}}{ }_{\mathrm{pke}}\right)$, where the function $g_{c^{*}, M \| r, 1^{\mu}{ }_{\mathrm{pke}}}(s k)$ uses the secret key to decrypt $c^{*}$, and signals whether the obtained plaintext is equal to $M \| r$ or to $1^{\mu_{\mathrm{pke}}}$.

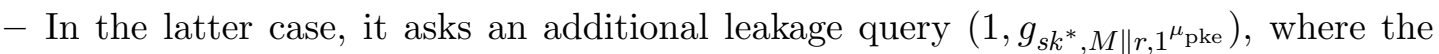

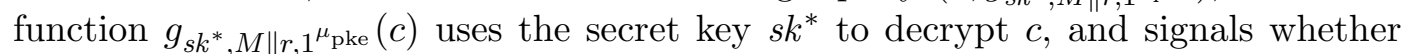
the obtained plaintext is equal to $M \| r$ or to $1^{\mu_{\text {pke }}}$.

Otherwise, the reduction returns a random guess $b^{\prime} \leftarrow\{0,1\}$.

Assume first that B is an $\ell^{\prime}$-admissible adversary (i.e., it does not violate the leakage bound), where the parameter $\ell^{\prime}$ is as in the statement of Theorem 1. Notice that, if $01 d_{0}$ happens, with probability $1-2^{-\kappa}$ due to the use of a pair-wise independent hash function, the extracted ciphertext $\widehat{c}$ obtained by the reduction is equal to one of the ciphertexts $c_{j}$ obtained by updating the original challenge ciphertext $c$; hence, in such a case, by correctness of the PKE scheme, adversary B wins with overwhelming probability. Furthermore, a similar argument shows that $B$ wins with overwhelming probability in case $01 d_{1}$ happens. On the other hand, if none of the events happen, $B$ is successful with probability $1 / 2$. Putting these observations together with the fact that $01 d$ happens if at least one of $01 d_{0}$ and $0 l d_{1}$ happen, we obtain

$$
\begin{aligned}
& 2\left|\mathbb{P}\left[\mathbf{G}_{\mathcal{P} \mathcal{K} \mathcal{E}, \mathrm{B}}^{\text {clrs }}\left(\kappa, \ell^{\prime}\right)=1\right]-\frac{1}{2}\right| \\
& =\left|\mathbb{P}\left[\mathbf{G}_{\mathcal{P} \mathcal{K} \mathcal{E}, \mathrm{B}}^{\text {clrs }}\left(\kappa, \ell^{\prime}\right)=1 \mid b=1\right]-\mathbb{P}\left[\mathbf{G}_{\mathcal{P} \mathcal{K} \mathcal{E}, \mathrm{B}}^{\text {clrs }}\left(\kappa, \ell^{\prime}\right)=0 \mid b=0\right]\right| \\
& =\mid \mathbb{P}[01 \mathrm{~d}] \cdot\left(\mathbb{P}\left[\mathbf{G}_{\mathcal{P} \mathcal{E} \mathcal{E}, \mathrm{B}}^{\text {clrs }}\left(\kappa, \ell^{\prime}\right)=1 \mid b=1,01 \mathrm{~d}\right]-\mathbb{P}\left[\mathbf{G}_{\mathcal{P} \mathcal{K}, \mathrm{B}}^{\text {clrs }}\left(\kappa, \ell^{\prime}\right)=0 \mid b=0,01 \mathrm{~d}\right]\right) \\
& \quad+\mathbb{P}[\overline{0 l d}] \cdot\left(\mathbb{P}\left[\mathbf{G}_{\mathcal{P} \mathcal{K} \mathcal{E}, \mathrm{B}}^{\text {clrs }}\left(\kappa, \ell^{\prime}\right)=1 \mid b=1, \overline{0 l d}\right]-\mathbb{P}\left[\mathbf{G}_{\mathcal{P} \mathcal{K}, \mathrm{B}}^{\mathrm{clrs}}\left(\kappa, \ell^{\prime}\right)=0 \mid b=0, \overline{0 l d}\right]\right) \mid \\
& \geq 1 / p_{3,4}(\kappa)-2 \cdot 2^{-\kappa},
\end{aligned}
$$

a contradiction.

It remains to show that B is $\ell^{\prime}$-admissible. Assume first that the event Old does not happen. Then, using an argument identical to that in the proof of the previous lemma, and considering 
that the reduction here additionally leaks two hash values at the beginning of each round, one can show that the composition of B's leakage functions is $\left(\ell+3 \mu+2 \kappa+\max \left\{\delta, \mu_{\text {pke }}\right\}\right)$-leaky. Thus, in order to conclude the proof, it suffices to show that the additional leakage due to the fact that the event 0ld happens does not reduce the entropy of the state further. While doing this, we distinguish the case where event $01 d_{0}$ and event $0 l d_{1}$ happen. In particular:

$01 d_{0}$ : We use the perfect secret-key-update privacy of the PKE scheme (cf. Definition 7). Let UpdateS $^{i}(\cdot):=$ UpdateS$\left._{\left(U_{p d a t e S}\right.}^{i-1}(\cdot)\right)$ if $i>1$, and UpdateS ${ }^{1}(\cdot):=U_{\text {updateS}}(\cdot)$. By Definition 7 , for any $(p, s)$ such that $\mathrm{PK}(s)=p$, the distribution $\{p, \operatorname{UpdateS}(s)\}$ is identical to $\left\{(p k, s k) \leftarrow \operatorname{KGen}\left(1^{\kappa}\right): p k=p\right\}$; this implies that for any $(p, s) \leftarrow \mathrm{KGen}\left(1^{\kappa}\right)$, if $s k$ is in the support of $\{$ UpdateS $(s)\}$, then $s k$ is also in the support of $\left\{s k^{\prime}:\left(p k^{\prime}, s k^{\prime}\right) \leftarrow \operatorname{KGen}\left(1^{\kappa}\right)\right.$, $\left.p k^{\prime}=p\right\}$. Therefore, by a simple inductive argument, we can show that for any $0 \leq i<i^{\prime}$ and any $(p, s) \leftarrow \mathrm{KGen}\left(1^{\kappa}\right)$, the distributions below are equivalent:

$$
\begin{aligned}
& \mathcal{D}_{i, i^{\prime}}^{p, s}:=\left\{\left(s k, s k^{\prime}\right): \begin{array}{c}
s k^{\prime} \leftarrow \operatorname{UpdateS}^{i}(s) \\
s k \leftarrow \operatorname{UpdateS}^{i^{\prime}}\left(s k^{\prime}\right)
\end{array}\right\} \\
& \tilde{\mathcal{D}}_{i, i^{\prime}}^{p, s}:=\left\{\left(s k, s k^{\prime}\right): \begin{array}{c}
p k^{\prime}=p ;\left(p k^{\prime}, s k^{\prime}\right) \leftarrow \operatorname{KGen}\left(1^{\kappa}\right) \\
s k \leftarrow \operatorname{UpdateS}^{i+i^{\prime}}(s)
\end{array}\right\} .
\end{aligned}
$$

Let now $i^{*}$ be the first index where $0 l \mathrm{~d}_{0}$ happens, and let $\mathrm{P}^{\prime}$ be an unbounded predictor. Then, for any $i<i^{*}$, we get:

$$
\begin{aligned}
\widetilde{\mathbb{H}}_{\infty}\left(s k_{i^{*}} \mid p k, s k_{i}\right) & \geq-\log \max _{\mathrm{P}^{\prime}} \mathbb{P}\left[\mathrm{P}^{\prime}\left(p k, s k_{i}\right)=s k_{i^{*}}: \begin{array}{c}
(p k, s k) \leftarrow \mathrm{KGen}\left(1^{\kappa}\right), \\
\left(s k_{i^{*}}, s k_{i}\right) \leftarrow \mathcal{D}_{i, i^{*}-i}^{p k, s k}
\end{array}\right] \\
& =-\log \max _{\mathrm{P}^{\prime}} \mathbb{P}\left[\mathrm{P}^{\prime}\left(p k, s k_{i}\right)=s k_{i^{*}}: \begin{array}{c}
(p k, s k) \leftarrow \mathrm{KGen}\left(1^{\kappa}\right) \\
\left(s k_{i^{*}}, s k_{i}\right) \leftarrow \tilde{\mathcal{D}}_{i, i^{*}-i}^{p k, s k}
\end{array}\right] \\
& =-\log \max _{\mathrm{P}^{\prime}} \mathbb{P}\left[\mathrm{P}^{\mathrm{P}^{\prime}}(p k)=s k_{i^{*}}: \quad(p k, s k) \leftarrow \mathrm{KGen}\left(1^{\kappa}\right)\right] \\
& \geq \widetilde{\mathbb{H}}_{\infty}\left(s k_{i^{*}} \mid p k\right),
\end{aligned}
$$

where $\mathrm{P}$ samples $s k_{i}$ from $\left\{\left(p k^{\prime}, s k^{\prime}\right) \leftarrow \mathrm{KGen}\left(1^{\kappa}\right): p k^{\prime}=p k\right\}$, and then runs $\mathrm{P}^{\prime}\left(p k, s k_{i}\right)$.

$0 \mathrm{ld}_{1}$ : We use the perfect ciphertext-update privacy property of the PKE scheme (cf. Definition 6). Let UpdateC $C^{i}(\cdot):=\operatorname{UpdateC}\left(\operatorname{Update}^{i-1}(\cdot)\right)$ if $i>1$, and $\operatorname{UpdateC}^{1}(\cdot):=$ UpdateC $(\cdot)$. By Definition 6 , for any $m \in \mathcal{M}_{\text {pke }}$ and $c$, such that $c=\operatorname{Enc}(p k, m ; r)$ for some $r \in\{0,1\}^{*}$, the distribution $\{p k, \operatorname{UpdateC}(c)\}$ is identical to $\{p k, \operatorname{Enc}(p k, m)\}$. This implies that for any $0 \leq i<i^{\prime}$, and any $(p k, s k) \leftarrow \operatorname{KGen}\left(1^{\kappa}\right)$, the distributions below are equivalent:

$$
\begin{aligned}
& \mathcal{D}_{i, i^{\prime}}^{p k, m}:=\left\{\left(c, c^{\prime}\right): \begin{array}{c}
\bar{c} \leftarrow \operatorname{Enc}(p k, m) ; c^{\prime} \leftarrow \operatorname{UpdateC}^{i}(\bar{c}) \\
c \leftarrow \operatorname{UpdateC} i^{\prime}\left(c^{\prime}\right)
\end{array}\right\} \\
& \tilde{\mathcal{D}}_{i, i^{\prime}}^{p k, m}:=\left\{\left(c, c^{\prime}\right): \quad \begin{array}{c}
\bar{c} \leftarrow \operatorname{Enc}(p k, m) ; c^{\prime} \leftarrow \operatorname{Enc}(p k, m) \\
c \leftarrow \operatorname{UpdateC}^{i+i^{\prime}}(\bar{c})
\end{array}\right\} .
\end{aligned}
$$

Let now $i^{*}$ be the first index where $01 \mathrm{~d}_{1}$ happens, and let $\mathrm{P}^{\prime}$ be an unbounded predictor. 
Then, for all $m \in \mathcal{M}_{\mathrm{pke}}$, and any $i<i^{*}$, we get:

$$
\begin{aligned}
\widetilde{\mathbb{H}}_{\infty}\left(c_{i^{*}} \mid p k, c_{i}\right) & \geq-\log \max _{\mathrm{P}^{\prime}} \mathbb{P}\left[\mathrm{P}^{\prime}\left(p k, c_{i}\right)=c_{i^{*}}: \begin{array}{c}
(p k, s k) \leftarrow \mathrm{KGen}\left(1^{\kappa}\right) \\
\left(c_{i^{*}}, c_{i}\right) \leftarrow \mathcal{D}_{i, i^{*}-i}^{p k, m}
\end{array}\right] \\
& =-\log \max _{\mathrm{P}^{\prime}} \mathbb{P}\left[\mathrm{P}^{\prime}\left(p k, c_{i}\right)=c_{i^{*}}: \begin{array}{c}
(p k, s k) \leftarrow \mathrm{KGen}\left(1^{\kappa}\right) \\
\left(c_{i^{*}}, c_{i}\right) \leftarrow \tilde{\mathcal{D}}_{i, i^{*}-i}^{p k, m}
\end{array}\right] \\
& =-\log \max _{\mathrm{P}^{\prime}} \mathbb{P}\left[\mathrm{P}^{\mathrm{P}^{\prime}}(p k)=c_{i^{*}}: \quad(p k, s k) \leftarrow \mathrm{KGen}\left(1^{\kappa}\right)\right] \\
& \geq \widetilde{\mathbb{H}}_{\infty}\left(c_{i^{*}} \mid p k\right),
\end{aligned}
$$

where $\mathrm{P}$ samples $c_{i}$ from $\{\operatorname{Enc}(p k, m)\}$, and then runs $\mathrm{P}^{\prime}\left(p k, c_{i}\right)$.

Lemma 7. For all $\kappa \in \mathbb{N}$, we have $\mathbf{G}_{\mathrm{A}}^{4}(\kappa) \equiv \mathbf{G}_{\mathrm{A}}^{5}(\kappa)$.

Proof. Recall that the simulator $\mathrm{S}_{1}$ returns same if and only if both tampering simulators $\mathrm{T}^{0}$ and $\mathrm{T}^{1}$ return $\diamond$; furthermore:

- The tampering simulator $\mathrm{T}^{0}$ returns $\diamond$ at round $i$ if either:

$-\widetilde{c} \in\left\{c, c_{i-1}, \ldots, c_{1}\right\}$; or

$-\widehat{c}=c$, and $\phi_{0}(\widehat{c})=\widetilde{c}$ (after running the extractor).

- The tampering simulator $\mathrm{T}^{1}$ returns $\diamond$ at round $i$ if either:

- $\widetilde{s k} \in\left\{s k, s k_{i-1}, \ldots, s k_{1}\right\}$; or

- $\widehat{s k}=s k$, and $\phi_{1}(\widehat{s k})=\widetilde{s k}$ (after running the extractor).

Let NotSame be the event that $\mathrm{S}_{1}$ outputs $\diamond$ in game $\mathbf{G}_{\mathrm{A}}^{4}(\kappa)$, but Decode $(\bar{\omega}, \widetilde{C}) \neq M$ in game $\mathbf{G}_{\mathrm{A}}^{5}(\kappa)$. Since the two games are identical conditioned on NotSame not happening, it suffices to bound the probability of event NotSame.

By definition of the set $\Phi_{1}$, we have that, in both cases where $\mathrm{T}^{1}$ returns $\diamond$, the secret key $s k$ is a valid secret key for the value $p k$. Similarly, by definition of the set $\Phi_{0}$, we have that, in both cases where $\mathrm{T}_{0}$ returns $\diamond$, the ciphertext $\widetilde{c}$ must meet the condition $\operatorname{Dec}(s k, \widetilde{c})=$ $\operatorname{Dec}(s k, c)$. Putting these observations together, we obtain that $\operatorname{Dec}(\widetilde{s k}, \widetilde{c})=\operatorname{Dec}(s k, c)=M \| r$, and thus event NotSame happens with zero probability (assuming the PKE scheme has perfect correctness). This concludes the proof of the claim.

Lemma 8. For all (even unbounded) adversaries $\mathrm{A}$, there exists a negligible function $\nu_{5,6}: \mathbb{N} \rightarrow$ $[0,1]$ such that $\left|\mathbb{P}\left[\mathbf{G}_{\mathbf{A}}^{5}(\kappa)=1\right]-\mathbb{P}\left[\mathbf{G}_{\mathbf{A}}^{6}(\kappa)=1\right]\right| \leq \nu_{5,6}(\kappa)$.

Proof. Game $\mathbf{G}_{\mathbf{A}}^{5}(\kappa)$ and $\mathbf{G}_{\mathbf{A}}^{6}(\kappa)$ are identical, unless there exists a tampering query such that both $\mathrm{T}^{0}$ and $\mathrm{T}^{1}$ return the same message $\widetilde{M} \notin\{\perp, \diamond\}$, but Decode $(\bar{\omega}, \widetilde{C}) \neq \widetilde{M}$ in game $\mathbf{G}_{\mathrm{A}}^{6}(\kappa)$. (This could happen also during the extra tampering query.) This means that the following situation happens in $\mathbf{G}_{\mathrm{A}}^{6}(\kappa)$ :

- $\operatorname{Dec}(\widehat{s k}, \widetilde{c})=\widetilde{M} \| \widetilde{r}$ and $\widetilde{\gamma}=\operatorname{Commit}(\omega, \widetilde{M} ; \widehat{r})$;

- $\operatorname{Dec}(\widetilde{s k}, \widetilde{c})=\widetilde{M}^{\prime} \| \widetilde{r}^{\prime}$ and $\widetilde{\gamma}=\operatorname{Commit}\left(\omega, \widetilde{M}^{\prime} ; \widetilde{r}^{\prime}\right)$, for some $\widetilde{M}^{\prime} \neq \widetilde{M}$. 
The above implies that there exist $(\widetilde{M}, \widehat{r}),\left(\widetilde{M}^{\prime}, \widetilde{r}^{\prime}\right)\left(\right.$ with $\left.\widetilde{M} \neq \widetilde{M}^{\prime}\right)$ such that $\widetilde{\gamma}=\operatorname{Commit}(\omega$, $\widetilde{M} ; \widehat{r})=\operatorname{Commit}\left(\omega, \widetilde{M}^{\prime} ; \widetilde{r}^{\prime}\right)$, which in turn contradicts the statistical binding property of the commitment scheme.

Lemma 9. For all PPT adversaries $\mathrm{A}$ there exists a negligible function $\nu_{6,7}: \mathbb{N} \rightarrow[0,1]$ such that $\left|\mathbb{P}\left[\mathbf{G}_{\mathrm{A}}^{6}(\kappa)=1\right]-\mathbb{P}\left[\mathbf{G}_{\mathrm{A}}^{7}(\kappa)=1\right]\right| \leq \nu_{6,7}(\kappa)$.

Proof. The proof is down to the label-malleable simulation extractability of the argument systems $\mathcal{N I}_{\mathcal{I}}$ and $\mathcal{N} \mathcal{I} \mathcal{A}_{1}$. For $\beta \in\{0,1\}$, let Abort $\beta$ be the event that $\mathrm{S}_{1}$ outputs Abort in game $\mathbf{G}_{\mathbf{A}}^{6}(\kappa)$ because $\mathbf{T}^{\beta}$ returned Abort as an answer to one of the tampering queries from the adversary. Define Abort $=$ Abort $_{0} \vee$ Abort ${ }_{1}$, and note that $\mathbf{G}_{A}^{6}(\kappa)$ and $\mathbf{G}_{A}^{7}(\kappa)$ are identical conditioned on Abort not happening. Thus, it suffices to bound the probability that Abort happens.

We start by proving that Abort $_{0}$ happens only with negligible probability. By contradiction, assume that there exists a PPT adversary $\mathrm{A}$ and a polynomial $p_{6,7}(\kappa)$, such that, for infinitely many values of $\kappa \in \mathbb{N}$, adversary $A$ provokes event Abort $_{0}$ with probability at least $1 / p_{6,7}(\kappa)$. Consider the following adversary $\mathrm{B}$ (with black-box access to $\mathrm{A}$ ), that is playing game $\mathbf{G}_{\mathcal{N} \mathcal{I} \mathcal{A}_{0}, \mathrm{~B}, \mathrm{~K}_{0}}^{\operatorname{lm}}\left(\kappa, \Phi_{0}\right)$.

Adversary B:

- Receive $\omega_{0}$ from the challenger. Run $\left(\omega_{1}, \tau_{\text {sim }}^{1}, \tau_{\text {ext }}^{1}\right) \leftarrow \mathrm{S}_{0}^{1}\left(1^{\kappa}\right)$, pick $\omega \leftarrow \operatorname{CRSGen}\left(1^{\kappa}\right)$, and $\rho \leftarrow \operatorname{Setup}\left(1^{\kappa}\right)$.

- Run $\mathrm{A}(\bar{\omega})$, where $\bar{\omega}:=\left(\omega_{0}, \omega_{1}, \omega, \rho\right)$, obtaining a message $M$.

- Compute the initial target encoding $\left(C_{0}, C_{1}\right)$ as $\mathrm{S}_{0}$ would do it in game $\mathbf{G}_{\mathrm{A}}^{7}(\kappa)$, except that the proof $\pi_{0}$ is obtained by forwarding $(c, p k)$ to the oracle $\mathcal{O}_{\text {sim }}^{*}(\cdot)$.

- Keep running $A$ by answering all of its queries exactly as $S_{1}$ would do it in game $\mathbf{G}_{\mathbf{A}}^{7}(\kappa)$, but with the following differences:

- Whenever $\mathrm{S}_{1}$ needs to run $\operatorname{Rfrsh}^{*}\left(\left(\tau_{\text {sim }}^{0}, \tau_{\text {sim }}^{1}\right),\left(C_{0}, C_{1}\right)\right)$, compute $C_{0}^{\prime}:=\left(p k, c^{\prime}, \pi_{0}^{\prime}\right)$ and $C_{1}^{\prime}:=\left(\gamma, s k^{\prime}, \pi_{1}^{\prime}\right)$ exactly as described in algorithm Rfrsh*, except that the proof $\pi_{0}^{\prime}$ is obtained by forwarding $\left(c^{\prime}, p k\right)$ to the oracle $\mathcal{O}_{\text {sim }}^{*}(\cdot)$;

- Whenever $\mathrm{S}_{1}$ returns Abort, upon input a tampering query $\left(f_{0}, f_{1}\right)$ from the adversary, ${ }^{11}$ compute $\left(\widetilde{p k}, \widetilde{c}, \widetilde{\pi}_{0}\right)=f_{0}\left(p k, c, \pi_{0}\right)$, and forward $(\widetilde{\lambda}, \widetilde{x}, \widetilde{\pi})$ to the challenger, where $\widetilde{\lambda}:=\widetilde{c}, \widetilde{x}:=\widetilde{p k}$, and $\widetilde{\pi}:=\widetilde{\pi}_{0}$.

By our assumption, with probability at least $1 / p_{6,7}(\kappa)$, the attacker $\mathrm{A}$ will provoke event $\mathrm{Abort}_{0}$. This means that, with the same probability, the tuple $(\widetilde{\lambda}, \widetilde{x}, \widetilde{\pi})$ returned by B satisfies the following:

- The proof $\widetilde{\pi}$ is accepting for the statement $\widetilde{x}$ w.r.t. the label $\widetilde{\lambda}$ (i.e., $\operatorname{Vrfy} \widetilde{c}\left(\omega_{0}, \widetilde{p k}, \widetilde{\pi}_{0}\right)=1$ ).

- The tuple $(\widetilde{\lambda}, \widetilde{x}, \widetilde{\pi})$ is not within the set $\mathcal{Q}$ of simulated proofs and corresponding statements/labels; this is because the tampering simulator extracts the proof only if $\left(\widetilde{p k}, \widetilde{c}, \widetilde{\pi}_{0}\right) \neq$ $\left(p k, c, \pi_{0}\right)$, and further it does not abort if $\widetilde{c} \in\left\{c_{1}, \ldots, c_{i-1}\right\}$ where $c_{1}, \ldots, c_{i-1}$ are the refreshed ciphertexts computed by the simulator $\mathrm{S}_{1}$ up to the $i$-th round.

- Either of the following conditions are met:

\footnotetext{
${ }^{11}$ Note that the pair $\left(f_{0}, f_{1}\right)$ might be either part of a (Tamp, $\left.\cdot\right)$ command or of a (Final, $\left.\cdot\right)$ command.
} 
(i) $\widehat{s k} \neq \perp$ and $(\widetilde{p k}, \widehat{s k}) \notin \mathcal{R}_{0}$; or

(ii) $\left(\phi_{0}, \widehat{c}\right) \neq(\perp)$ and either $\phi_{0} \notin \Phi_{0}$, or $(\widehat{c}, \widetilde{p k}) \notin \mathcal{Q}_{\downarrow},{ }^{12}$ or $\phi_{0}(\widehat{c}) \neq \widetilde{c}$; or

(iii) $\left(\widehat{s k}, \phi_{0}, \widehat{c}\right)=(\perp, \perp, \perp)$.

Hence, B breaks label-malleable simulation extractability of $\mathcal{N} \mathcal{I} \mathcal{A}_{0}$ with non-negligible probability, and thus Abort ${ }_{0}$ only happens with a negligible probability. A similar argument (omitted here) shows that Abort ${ }_{1}$ also happens with negligible probability. The proof of the lemma now follows by an application of the union bound.

Lemma 10. For all (even unbounded) adversaries $\mathrm{A}$, there exists a negligible function $\nu_{7,8}$ : $\mathbb{N} \rightarrow[0,1]$ such that $\left|\mathbb{P}\left[\mathbf{G}_{\mathbf{A}}^{7}(\kappa)=1\right]-\mathbb{P}\left[\mathbf{G}_{\mathbf{A}}^{8}(\kappa)=1\right]\right| \leq \nu_{7,8}(\kappa)$.

Proof. We rely on the statistical binding property of the commitment and the correctness property of the PKE scheme. Assume $\left(f_{0}, f_{1}\right)$ be any tampering query produced by the adversary, such that Decode $\left(\bar{\omega},\left(f_{0}\left(C_{0}\right), f_{1}\left(C_{1}\right)\right)\right)=\widetilde{M} \neq \perp$ in game $\mathbf{G}_{\mathrm{A}}^{8}(\kappa)$, but the simulator $\mathrm{S}_{1}$ of game $\mathbf{G}_{\mathrm{A}}^{7}(\kappa)$ instead would return $\perp$. Further, let $\widetilde{M}_{0}$ and $\widetilde{M}_{1}$ the values returned by the tampering simulators $\mathrm{S}_{0}$ and $\mathrm{S}_{1}$, respectively, upon input the tampering query $\left(f_{0}, f_{1}\right)$. We distinguish two cases.

Case 1: $\widetilde{M}_{0} \neq \widetilde{M}_{1}$ and $\widetilde{M}_{0}, \widetilde{M}_{1} \neq \perp$. We claim that $\widetilde{M}=\widetilde{M}_{0}$. In fact, the decoding algorithm decrypts $\widetilde{c}$ using $\widetilde{s k}$, whereas $\mathrm{T}^{0}$ decrypts $\widetilde{c}$ using $\widehat{s k}$, but $\operatorname{PK}(\widetilde{s k})=\operatorname{PK}(\widehat{s k})$. Therefore, by the correctness of the PKE scheme, we must have $\widetilde{M}=\widetilde{M}_{0}$.

On the other hand, the decoding algorithm also checks that $\widetilde{\gamma}$ is a valid commitment to $\widetilde{M}$, and $\mathrm{T}^{1}$ extracts $\widehat{M}=\widetilde{M}_{1}$ as a valid opening of $\widetilde{\gamma}$. This contradict the statistical binding property of the commitment scheme.

Case 2: At least one of $\widetilde{M}_{0}, \widetilde{M}_{1}$ is equal to $\perp$. Note that the tampering simulator $\mathrm{T}^{0}$ (resp., $\mathrm{T}^{1}$ ) returns $\perp$ only in case the proof $\widetilde{\pi}_{0}$ (resp., $\widetilde{\pi}_{1}$ ) is invalid. However, in such a case also the decoding algorithm outputs $\perp$, and thus we conclude that $\widetilde{M}=\perp$ (a contradiction).

Lemma 11. For all $\kappa \in \mathbb{N}$, and for all (even unbounded) adversaries $\mathrm{A}$, we have $\mathbf{G}_{\mathrm{A}}^{8}(\kappa) \equiv$ $\mathbf{G}_{\mathrm{A}}^{9}(\kappa)$.

Proof. The only difference between the two games is that, while answering the extra tampering query, the former game computes $s k^{\prime}$ as a refresh of the extracted secret key $\widehat{s k}_{0}$, whereas the latter game computes $s k^{\prime}$ as refresh of the tampered secret key $\widetilde{s k}$. However, note that the simulator $S_{1}$ runs the extractor $K_{0}$ on the same random coins as used to obtain the messages $\widetilde{M}_{0}^{*}$ and $\widetilde{M}_{1}^{*}$. Since $\mathrm{S}_{1}$ never aborts in game $\mathbf{G}_{\mathrm{A}}^{8}(\kappa)$, and since we are looking at the case $\widetilde{M}_{0}^{*}=\widetilde{M}_{1}^{*} \notin\{\perp, \diamond\}$, we conclude that $\widetilde{s k}$ is a valid secret key corresponding to $p k$. Hence, the lemma directly follows by the secret-key update privacy property of the PKE scheme.

Lemma 12. For all $\kappa \in \mathbb{N}$, and for all (even unbounded) adversaries $\mathrm{A}$, we have $\mathbf{G}_{\mathrm{A}}^{9}(\kappa) \equiv$ $\mathbf{G}_{\mathrm{A}}^{10}(\kappa)$.

\footnotetext{
${ }^{12}$ The fact that $(\widehat{c}, \widetilde{p k}) \notin \mathcal{Q}_{\downarrow}$ follows by the fact that $\mathrm{T}^{0}$ outputs Abort in step $2 \mathrm{~d}$ of game $\mathbf{G}_{\mathrm{A}}^{7}(\kappa)$ only if $\widehat{c} \notin\left\{c_{1}, \ldots, c_{i-1}\right\}$, where $c_{1}, \ldots, c_{i-1}$ are the refreshed ciphertexts computed by the simulator $\mathrm{S}_{1}$ up to the $i$-th round; furthermore, in case $\widehat{c}=c_{i}$, the tampering simulator does not abort.
} 
Proof. The only difference between the two games is that, while answering the extra tampering query, the former game computes $c^{\prime}$ as a fresh encryption of the extracted value $\widehat{M} \| \widehat{r}$, whereas the latter game computes $c^{\prime}$ as refresh of the tampered ciphertext $\widetilde{c}$. However, note that the simulator $S_{1}$ runs the extractor $K_{1}$ on the same random coins as used to obtain the messages $\widetilde{M}_{0}^{*}$ and $\widetilde{M}_{1}^{*}$. Since $\mathrm{S}_{1}$ never aborts in game $\mathbf{G}_{\mathrm{A}}^{9}(\kappa)$, and since we are looking at the case $\widetilde{M}_{0}^{*}=\widetilde{M}_{1}^{*} \notin\{\perp, \diamond\}$, we conclude that $\widetilde{c}$ is a valid encryption of the value $\widehat{M} \| \widehat{r}$. Hence, the lemma directly follows by the ciphertext update privacy property of the PKE scheme.

Lemma 13. For all PPT adversaries $\mathrm{A}$ there exists a negligible function $\nu_{10,11}: \mathbb{N} \rightarrow[0,1]$ such that $\left|\mathbb{P}\left[\mathbf{G}_{\mathrm{A}}^{10}(\kappa)=1\right]=\mathbb{P}\left[\mathbf{G}_{\mathrm{A}}^{11}(\kappa)=1\right]\right| \leq \nu_{10,11}(\kappa)$.

Proof. The only difference between the two games is that in the former game all proofs for the relation $\mathcal{R}_{0}$ are computed via the zero-knowledge simulator $S_{1}^{0}$, whereas in the latter game such proofs are computed by running the real prover algorithm Prove $_{0}$. Hence, the lemma follows by a straightforward reduction to the adaptive multi-theorem property of $\mathcal{N} \mathcal{I} \mathcal{A}_{0}$.

Lemma 14. For all PPT adversaries $\mathrm{A}$ there exists a negligible function $\nu_{11,12}: \mathbb{N} \rightarrow[0,1]$ such that $\left|\mathbb{P}\left[\mathbf{G}_{\mathrm{A}}^{11}(\kappa)=1\right]=\mathbb{P}\left[\mathbf{G}_{\mathrm{A}}^{12}(\kappa)=1\right]\right| \leq \nu_{11,12}(\kappa)$.

Proof. Identical to the proof of the previous lemma, but we now reduce to the (adaptive multitheorem) zero-knowledge property of $\mathcal{N} \mathcal{I} \mathcal{A}_{1}$.

Lemma 15. For all PPT adversaries $\mathrm{A}$ there exists a negligible function $\nu_{12,13}: \mathbb{N} \rightarrow[0,1]$ such that $\left|\mathbb{P}\left[\mathbf{G}_{\mathrm{A}}^{12}(\kappa)=1\right]=\mathbb{P}\left[\mathbf{G}_{\mathrm{A}}^{13}(\kappa)=1\right]\right| \leq \nu_{12,13}(\kappa)$.

Proof. The only difference between the two games is that in the former game, while refreshing a codeword, the proof corresponding to relation $\mathcal{R}_{0}$ is computed by running the prover algorithm Prove $_{0}$, whereas in the latter game such proof is obtained by running the label evaluation procedure LEval ${ }_{0}$. Hence, the lemma follows by a straightforward reduction to the label derivation privacy of $\mathcal{N} \mathcal{I} \mathcal{A}_{0}$.

Lemma 16. For all PPT adversaries $\mathrm{A}$ there exists a negligible function $\nu_{13,14}: \mathbb{N} \rightarrow[0,1]$ such that $\left|\mathbb{P}\left[\mathbf{G}_{\mathrm{A}}^{13}(\kappa)=1\right]=\mathbb{P}\left[\mathbf{G}_{\mathrm{A}}^{14}(\kappa)=1\right]\right| \leq \nu_{13,14}(\kappa)$.

Proof. Identical to the proof of the previous lemma, but we now reduce to the label derivation privacy property of $\mathcal{N} \mathcal{I} \mathcal{A}_{1}$.

\section{Concrete Instantiations}

For a group $\mathbb{G}$ of prime order $q$ and a generator $g$ of $\mathbb{G}$, we denote by $[a]_{g}:=g^{a} \in \mathbb{G}$ the implicit representation of an element $a \in \mathbb{Z}_{q}$. Let $\mathrm{G}$ be a PPT pairing generation algorithm that, upon input the security parameter $1^{\kappa}$, outputs a tuple params $=\left(\mathbb{G}_{1}, \mathbb{G}_{2}, \mathbb{G}_{T}, q, g, h\right.$, ê $)$ where the first three elements are the description of groups of prime order $q>2^{\kappa}, g$ (resp. $h$ ) is a generator for the group $\mathbb{G}_{1}$ (resp. $\mathbb{G}_{2}$ ), and ê : $\mathbb{G}_{1} \times \mathbb{G}_{2} \rightarrow \mathbb{G}_{T}$ is an efficiently computable non-degenerate pairing function.

Within this section, vectors and matrices are denoted boldface, and vectors are intended as row vectors. Given a group $\mathbb{G}$, two matrices $\mathbf{X} \in \mathbb{G}^{n \times m}$ and $\mathbf{Y} \in \mathbb{G}^{m \times t}$, for some $n, m, t \geq 1$, and an element $a \in \mathbb{G}$, we denote by $\mathbf{X} \cdot \mathbf{Y}$ the matrix product of $\mathbf{X}$ and $\mathbf{Y}$, and by $a \cdot \mathbf{X}$ the scalar multiplication of $\mathbf{X}$ by $a$. Given two elements $[a]_{g} \in \mathbb{G}_{1}$ and $[b]_{h} \in \mathbb{G}_{2}$, we denote by $[a]_{g} \bullet[b]_{h}=[a \cdot b]_{e(g, h)}$ the value $\hat{\mathrm{e}}\left([a]_{g},[b]_{h}\right)$; this notation is also applied to vectors and matrices in the natural way. Given a field $\mathbb{F}$ and natural numbers $n, m, j \in \mathbb{N}$, where $j \leq \min (n, m)$, we define $\mathrm{Rk}_{j}\left(\mathbb{F}^{n \times m}\right)$ to be the set of matrices in $\mathbb{F}^{n \times m}$ with row rank $j$; given a matrix $\mathbf{B}$ we let $\operatorname{Rank}(\mathbf{B})$ be the rank of $\mathbf{B}$. 
Definition 13 ( $k$-Rank hiding assumption). Let $\mathrm{G}$ be a pairing generation algorithm as above. The $k$-rank hiding assumption holds for $\mathrm{G}$ if, for any $k \leq j, j^{\prime} \leq \min (n, m)$, we have that $\left(g,[\mathbf{B}]_{g}\right) \approx_{c}\left(g,\left[\mathbf{B}^{\prime}\right]_{g}\right)$ and $\left(h,[\mathbf{B}]_{h}\right) \approx_{c}\left(h,\left[\mathbf{B}^{\prime}\right]_{h}\right)$, where $\mathbf{B} \leftarrow \mathrm{Rk}_{j}, \mathbf{B}^{\prime} \leftarrow \mathrm{Rk}_{j^{\prime}}$, and $\left(\mathbb{G}_{1}, \mathbb{G}_{2}, \mathbb{G}_{T}\right.$, $q, g, h, \hat{\mathrm{e}}) \leftarrow \mathrm{G}\left(1^{\kappa}\right)$.

As shown by Naor and Segev in [51], the $k$-rank hiding assumption follows from the more common $k$-linear assumption. The assumption gets weaker as $k$ increases; in fact, for $k=1$, this assumption is equivalent to the DDH assumption. Unfortunately, DDH does not hold in symmetric pairing groups where $\mathbb{G}_{1}=\mathbb{G}_{2}$. However, it is widely believed that DDH still holds in asymmetric pairing groups; this is sometimes known as the external Diffie-Hellman (SXDH) assumption [11].

\subsection{The PKE Scheme}

We consider the CLRS Friendly PKE scheme of [33] (which in turn is based on the one in [29]). Consider the following PKE scheme $\mathcal{P K \mathcal { E }}=$ (Setup, KGen, Enc, Dec, UpdateC, UpdateS), with message space $\mathcal{M}:=\{0,1\}$ and parameters $n, m, d \in \mathbb{N}$.

- Setup $\left(1^{\kappa}\right)$ : Sample params $\leftarrow \mathrm{G}\left(1^{\kappa}\right)$, and vectors $\mathbf{p}, \mathbf{w} \leftarrow \mathbb{Z}_{q}^{m}$ such that $\mathbf{p} \cdot \mathbf{w}^{\mathrm{T}}=0 \bmod q$. $\overline{\text { Return } \rho:=}$ (params, $\left.[\mathbf{p}]_{g},[\mathbf{w}]_{h}\right)$. (Recall that all algorithms implicitly take $\rho$ as input.)

- $\underline{\mathrm{KGen}}(\rho)$ : Sample $\mathbf{t} \leftarrow \mathbb{Z}_{q}^{m}, \mathbf{r} \leftarrow \mathbb{Z}_{q}^{n}$ and compute $s k:=\left[\mathbf{r}^{\mathrm{T}} \cdot \mathbf{w}+\mathbf{1}_{n}^{\mathrm{T}} \cdot \mathbf{t}\right]_{h}$, set $\alpha:=\mathbf{p} \cdot \mathbf{t}^{\mathrm{T}}$ and compute $p k:=[\alpha]_{g}$. (The latter can be done given only $[\mathbf{p}]_{g} \in \mathbb{G}_{1}^{m}$ and $\mathbf{t} \in \mathbb{Z}_{q}^{m}$.) Return $(p k, s k)$.

- Enc $(p k, b):$ Sample $\mathbf{u} \leftarrow \mathbb{Z}_{q}^{n}$ and compute $c_{1}:=\left[\mathbf{u}^{\mathrm{T}} \cdot \mathbf{p}\right]_{g}$ and $c_{2}:=\left[\alpha \mathbf{u}+b \mathbf{1}_{n}\right]_{g}$. Return $\bar{c}:=\left(c_{1}, c_{2}\right)$.

- $\operatorname{Dec}(s k, C)$ : Let $f=\hat{\mathrm{e}}(g, h)$, parse $s k=[\mathbf{S}]_{h} \in \mathbb{G}_{2}^{n \times m}$, let $\mathbf{S}_{1}$ be the first row of $\mathbf{S}$, and parse $c=\left([\mathbf{C}]_{g},[\mathbf{c}]_{g}\right) \in \mathbb{G}_{1}^{n \times m} \times \mathbb{G}_{1}^{n}$. Compute $\mathbf{b}:=\left[\mathbf{c}-\mathbf{C} \cdot \mathbf{S}_{1}^{\mathrm{T}}\right]_{f}$ and output 1 if and only if $\mathbf{b}=\left[\mathbf{1}_{n}\right]_{f}$. Note that $[\mathbf{b}]_{f}$ can be determined by first computing $[\mathbf{c}]_{f}:=\hat{\mathrm{e}}\left([\mathbf{c}]_{g}, h\right)$, and then $\left[\mathbf{C} \cdot \mathbf{S}_{1}^{\mathrm{T}}\right]_{f}:=\prod_{i} \hat{\mathrm{e}}(\mathbf{C}[i], \mathbf{S}[i])$.

- UpdateC $(C)$ : Parse $c=\left([\mathbf{C}]_{g},[\mathbf{c}]_{g}\right) \in\left(\mathbb{G}_{1}^{n \times m} \times \mathbb{G}_{1}^{n}\right)$. Sample $\mathbf{B} \leftarrow \mathbb{Z}_{q}^{n \times n}$ such that $\mathbf{B} \cdot \mathbf{1}_{n}^{\mathrm{T}}=$ $\mathbf{1}_{n}$ and the rank of $\mathbf{B}$ is $d$. Return $\left([\mathbf{B} \cdot \mathbf{C}],\left[\mathbf{B} \cdot \mathbf{c}^{\mathrm{T}}\right]\right)$.

- UpdateS(sk): Parse $s k=[\mathbf{S}]_{h} \in \mathbb{G}_{2}^{n \times m}$. Sample $\mathbf{A} \leftarrow \mathbb{Z}_{q}^{n \times n}$ such that $\mathbf{A} \cdot \mathbf{1}_{n}^{\mathrm{T}}=\mathbf{1}_{n}$ and the rank of $\mathbf{A}$ is $d$. Return $[\mathbf{A} \cdot \mathbf{S}]_{h}$.

Faonio and Nielsen [33] showed that, under the SXDH assumption, the above PKE scheme is CLRS-friendly in the bounded leakage model (i.e., the total amount of leakage is upper bounded by a value $\ell \in \mathbb{N}$ ). The theorem below states that the same PKE is also secure in the more general noisy leakage model.

Theorem 2. For any $m \geq 6, n \geq 3 m-6$, and $d:=n-m+3$, the above construction gives an $\ell$-noisy CLRS-friendly PKE scheme under the SXDH assumption, for $\ell=\min \{m / 6-1, n-$ $3 m+6\} \cdot \log (q)-\omega(\log \kappa)$.

Proof sketch. The proof is heavily based on the elegant proof in [29]. Most of the hybrids are identical. The main difference is that we need a "noisy version" of the so-called subspace hiding lemma (cf. Lemma B7 in [29]). We state this lemma below. 
Lemma 17 (Noisy model version of Lemma B.7 of [29]). Let the integers $d, n, s, u$ be polynomials in the security parameter $\kappa \in \mathbb{N}$. Let $\mathbf{S} \in \mathbb{Z}_{q}^{d \times s}$ be an arbitrary (fixed and public) matrix, and $L$ an $\ell$-leaky function (possibly chosen depending on $\mathbf{S}$ ). For randomly sampled $\mathbf{A} \leftarrow \mathbb{Z}_{q}^{n \times d}$, $\mathbf{V} \leftarrow \mathbb{Z}_{q}^{d \times u}$, and $\mathbf{U} \leftarrow \mathbb{Z}_{q}^{n \times u}$, we have:

$$
(L(\mathbf{A}), \mathbf{A S}, \mathbf{V}, \mathbf{A V}) \approx_{s}(L(\mathbf{A}), \mathbf{A S}, \mathbf{V}, \mathbf{U}),
$$

as long as $(d-s-u) \log (q)-\ell=\omega(\log \kappa)$.

Proof sketch. The proof follows almost verbatim the one in [29]. In particular, it suffices to apply the leftover-hash lemma for leaky sources [30] to each row of $\mathbf{A}$ independently. Take any row $\mathbf{a}_{i}$ of $\mathbf{A}$, and think of it as a random source whose conditional average min-entropy is

$$
\widetilde{\mathbb{H}}_{\infty}\left(\mathbf{a}_{i} \mid \mathbf{A S}, L(\mathbf{A})\right) \geq \widetilde{\mathbb{H}}_{\infty}\left(\mathbf{a}_{i} \mid L(\mathbf{A})\right)-s \log (q) \geq d \log (q)-s \log (q)-\ell,
$$

where, in the second step, we simply applied the definition of an $\ell$-leaky function (notice that $\mathbf{A}$ is uniform). Now, think of $\mathbf{V}$ as the seed of the universal hash function $h_{\mathbf{V}}\left(\mathbf{a}_{i}\right)=\mathbf{a}_{i} \mathbf{V}$, whose output size is $u \log (q)$ bits. The leftover-hash lemma tells us that the $i$ th row of $\mathbf{A V}$ looks uniform. By using the hybrid argument over all the rows we obtain the lemma.

For any $k \in \mathbb{N}$, let $\mathcal{P} \mathcal{K} \mathcal{E}^{\times k}=\left(\right.$ Setup, KGen, Enc $\left.{ }^{\times k}, \operatorname{Dec}^{\times k}\right)$ where $\operatorname{Enc}^{\times k}\left(p k, m_{1}, \ldots, m_{k}\right):=$ $\left(\operatorname{Enc}\left(p k, m_{1}\right), \operatorname{Enc}\left(p k, m_{2}\right), \ldots, \operatorname{Enc}\left(p k, m_{k}\right)\right)$, and $\operatorname{Dec}{ }^{\times k}$ performs the obvious decryption procedure. The lemma below says that constructing single-bit PKE is sufficient for obtaining $k$-bit $\mathrm{PKE}$, in the setting of CLRS-friendly security.

Lemma 18. For any polynomial $k(\kappa)$, if $\mathcal{P K \mathcal { E }}$ is an $\ell$-noisy $C L R S$-friendly secure PKE, then $\mathcal{P K} \mathcal{E}^{\times k}$ is an $\ell$-noisy CLRS-friendly secure PKE.

\subsection{The Commitment Scheme}

We use the commitment scheme of [43], that is secure under the 2-LIN (a.k.a. DLIN) assumption. The scheme depends on some global parameters, that for simplicity we consider to be the same as the ones for the PKE scheme from the previous section. Let $\psi: \mathbb{Z}_{q}^{2} \rightarrow \mathbb{Z}_{q}^{3}$ be such that $\psi\left(r_{1}, r_{2}\right)=\left(r_{1}, r_{2}, r_{1}+r_{2}\right)$.

- Setup $\left(1^{\kappa}\right)$ : Sample $\mathbf{p} \leftarrow \mathbb{Z}_{q}^{3}$, and output $\omega=[\mathbf{x}]_{g}$.

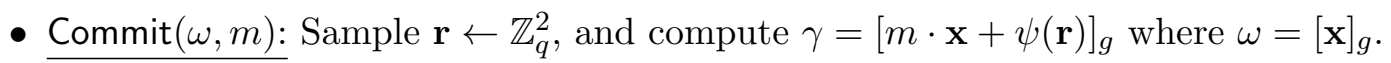

\subsection{The Label-Malleable NIZK}

In [33], the authors show that lm-NIZK are a special kind of Controlled-Malleable NIZK (cmNIZK) argument of knowledge systems [15], and provide a generic transformation to obtain lm-NIZK. In particular, their paradigm identifies a sufficient set of conditions which we review below.

Definition 14. For a relation $\mathcal{R}$, and a set of transformations $\Phi$ on the set of labels $\Lambda$, we say $(\mathcal{R}, \Phi)$ is label-malleable friendly if the following properties hold.

1. Representable statements and labels: Any instance and witness of $\mathcal{R}$ can be represented as a set of group elements; i.e., there are efficiently computable bijections $F_{\text {stm }}: \mathcal{L}_{\mathcal{R}} \rightarrow$ $\mathbb{G}_{i_{\text {stm }}}^{d_{\text {stm }}}$ for some $d_{\text {stm }}$ and $i_{\text {stm }}, F_{\text {wit }}: \mathcal{W}_{\mathcal{R}} \rightarrow G_{i_{\text {wit }}}^{d_{\text {wit }}}$ for some $d_{\text {wit }}$ and $i_{\text {wit }}$, and where $\mathcal{W}_{\mathcal{R}}$ is the witness space for the relation $\mathcal{R}$, and $F_{\mathrm{lbl}}: \Lambda \rightarrow \mathbb{G}_{i_{\mid \mathrm{bl}}}^{d_{\mathrm{bl}}}$ for some $d_{\mathrm{lbl}}$ and $i_{\mathrm{lbl}}=i_{\mathrm{stm}}$. 
2. Representable transformations: Any transformation in $\Phi$ can be represented as a set of group elements; i.e., there is an efficiently computable bijection $F_{\text {trs }}: \Phi \rightarrow G_{i_{\text {trs }}}^{d_{\text {trs }}}$ for some $d_{\text {trs }}$ and some $i_{\text {trs }}$.

3. Provable statements: We can prove the statement " $(x, w) \in \mathcal{R}$ " (using the above representation for $x$ and $w$ ) via pairing product equations; i.e., there is a pairing product statement that is satisfied by $F_{\text {stm }}(x)$ and $F_{\text {wit }}(w)$ if and only if $(x, w) \in \mathcal{R}$.

4. Provable transformations: We can prove the statement " $\phi\left(\lambda^{\prime}\right)=\lambda \wedge \phi \in \Phi$ " (using the above representations for labels $\lambda, \lambda^{\prime}$ and transformation $\phi$ ) via a pairing product equation, i.e., there is a pairing product statement that is satisfied by $F_{\mathrm{trs}}(\phi), F_{\mid \mathrm{bl}}(\lambda), F_{\mid \mathrm{bl}}\left(\lambda^{\prime}\right)$ if and only if $\phi \in \Phi \wedge \phi\left(\lambda^{\prime}\right)=\lambda$.

5. Transformable transformations: For any $\phi, \phi^{\prime} \in \Phi$, there is an efficient transformation (depending on $\phi, \phi^{\prime}$ ) that takes the statement " $\phi\left(\lambda^{\prime}\right)=\lambda \wedge \phi \in \Phi$ " (phrased using pairing product equations as above) and transforms it into the statement " $\left(\phi^{\prime} \circ \phi\right)\left(\lambda^{\prime}\right)=$ $\phi(\lambda) \wedge\left(\phi^{\prime} \circ \phi\right) \in \Phi "$ while preserving the label $\lambda^{\prime}$.

Theorem 3 (Theorem 3 of [33]). If the DLIN assumption holds, then we can construct a lMNIZK with label derivation privacy from any label-malleable friendly relation and transformation $\operatorname{set}(\mathcal{R}, \Phi)$.

Consider now the relation and transformation set $\left(\mathcal{R}_{\rho}^{0}, \Phi_{\rho}^{0}\right)$ defined below:

$$
\begin{aligned}
\mathcal{R}_{\rho}^{0} & =\left\{\left([\alpha]_{g},[\mathbf{S}]_{h}\right):[\alpha]_{g}=\left[\mathbf{p} \cdot \mathbf{S}_{1}^{\mathrm{T}}\right]_{g}\right\} \\
\Phi_{\rho}^{0} & =\left\{\phi_{\mathbf{B}}(\mathbf{C}, \mathbf{c}):=\left(\left[\mathbf{B} \cdot \mathbf{C}^{\mathrm{T}}\right]_{g},\left[\mathbf{B} \cdot \mathbf{c}^{\mathrm{T}}\right]_{g}\right): \mathbf{1}=\mathbf{B} \cdot \mathbf{1}^{\mathrm{T}}\right\} .
\end{aligned}
$$

where $\rho=$ (params, $\left.[\mathbf{p}]_{g},[\mathbf{w}]_{h}\right) \leftarrow \operatorname{Setup}\left(1^{\kappa}\right)$ is as in the PKE scheme from Section 5.1. Notice that the set of all the possible updates of a ciphertext, i.e.

$$
\left\{\phi: \phi(\cdot)=\operatorname{UpdateC}(\rho, \cdot ; \mathbf{B}), \mathbf{B} \in \mathbb{Z}_{q}^{n \times n}, \mathbf{1}_{n}=\mathbf{B} \cdot \mathbf{1}_{n}^{\mathrm{T}}, \operatorname{rank}(\mathbf{B})=d\right\},
$$

is a subset of $\Phi_{\rho}^{0}$. We show that $\left(\mathcal{R}_{\rho}^{0}, \Phi_{\rho}^{0}\right)$ is label-malleable friendly.

Representable statements and labels: Notice that $\mathcal{L}_{\mathcal{R}_{\rho}^{0}} \subseteq \mathbb{G}_{1}$, while the set of valid labels is equal to $\mathbb{G}_{1}^{n \times m} \times \mathbb{G}_{1}^{n}$.

Representable transformations: We can describe a transformation $\phi_{\mathbf{B}} \in \Phi_{\rho}^{0}$ as a matrix of elements $[\mathbf{B}]_{h} \in \mathbb{G}_{2}^{n \times n}$.

Provable statements: The relation $\mathcal{R}_{\rho}^{0}$ can be represented by the pairing product statement $[\alpha]_{g} \bullet[1]_{h}=[\mathbf{p}] \bullet\left[\mathbf{S}_{1}^{\mathrm{T}}\right]_{h}$.

Provable transformations: Given a transformation $\phi_{\mathbf{B}} \in \Phi_{\rho}^{0}$ and labels $c=\left([\mathbf{C}]_{g},[\mathbf{c}]_{g}\right), c^{\prime}=$ $\left(\left[\mathbf{C}^{\prime}\right]_{g},\left[\mathbf{c}^{\prime}\right]_{g}\right)$, the statement " $\phi_{\mathbf{B}}\left(c^{\prime}\right)=c \wedge \phi_{\mathbf{B}} \in \Phi$ " can be expressed as a system of pairing product equations:

$$
\left\{\begin{array}{l}
{[\mathbf{B}]_{h} \bullet\left[\mathbf{C}^{\prime \mathrm{T}}\right]_{g}=[\mathbf{C}]_{g} \bullet[1]_{h}} \\
{[\mathbf{B}]_{h} \bullet\left[\mathbf{c}^{\prime \mathrm{T}}\right]_{g}=[\mathbf{c}]_{g} \bullet[1]_{h}} \\
{[\mathbf{B}]_{h} \bullet\left[\mathbf{1}^{\mathrm{T}}\right]_{g}=[\mathbf{1}]_{f}}
\end{array}\right.
$$

Transformable transformations: Let $\phi_{\mathbf{B}}, c, c^{\prime}$ be as before, and let $\phi_{\mathbf{B}^{\prime}} \in \Phi_{\rho}^{0}$. We show that we can transform the system in Eq. (8) into a system of pairing product equations 
corresponding to the statement $\left(\phi_{\mathbf{B}^{\prime}} \circ \phi_{\mathbf{B}}\right)\left(c^{\prime}\right)=\phi_{\mathbf{B}^{\prime}}(c) \wedge\left(\phi_{\mathbf{B}^{\prime}} \circ \phi_{\mathbf{B}}\right) \in \Phi$. In particular, we obtain

$$
\left\{\begin{array}{l}
{\left[\mathbf{B}^{\prime} \cdot \mathbf{B}\right]_{h} \bullet\left[\mathbf{C}^{\prime \mathrm{T}}\right]_{g}=\left[\mathbf{B}^{\prime} \cdot \mathbf{C}^{\mathrm{T}}\right]_{g} \bullet[1]_{h}} \\
{\left[\mathbf{B}^{\prime} \cdot \mathbf{B}\right]_{h} \bullet\left[\mathbf{c}^{\prime \mathrm{T}}\right]_{g}=\left[\mathbf{B}^{\prime} \cdot \mathbf{c}^{\mathrm{T}}\right]_{g} \bullet[1]_{h}} \\
{\left[\mathbf{B}^{\prime} \cdot \mathbf{B}\right]_{h} \bullet\left[\mathbf{1}^{\mathrm{T}}\right]_{g}=[\mathbf{1}]_{f} .}
\end{array}\right.
$$

For any $k \in \mathbb{N}$, consider the PKE scheme $\mathcal{P} \mathcal{K E}^{\times k}$ defined in Section 5.1, let $\mathcal{C}_{\mathcal{P} K \mathcal{E}^{\times k}}=\left(\mathcal{C}_{\mathcal{P K E}}\right)^{k}$ be the ciphertext space of $\mathcal{P} \mathcal{K} \mathcal{E}^{\times k}$, and $\Phi_{\rho}^{0^{\times k}}=\left(\Phi_{\rho}^{0}\right)^{k}$. One can easily show that, for any positive polynomial $k(\kappa)$, the tuple $\left(\mathcal{R}_{\rho}^{0}, \Phi_{\rho}^{0^{\times k}}\right)$ is label-malleable friendly.

Next, consider the relation and transformation set $\left(\mathcal{R}_{\rho}^{1}, \Phi_{\rho}^{1}\right)$ defined below:

$$
\begin{aligned}
\mathcal{R}_{\rho}^{1} & =\left\{\left([\mathbf{x}]_{g},[\alpha]_{g}\right),(m, \mathbf{r}):[\alpha]_{g}=[m \cdot \mathbf{x}+\psi(\mathbf{r})]_{g}\right\} \\
\Phi_{\rho}^{1} & =\left\{\phi_{\mathbf{A}}(\mathbf{S}):=\left(\left[\mathbf{A} \cdot \mathbf{S}^{\mathrm{T}}\right]_{h}\right): \mathbf{1}=\mathbf{A} \cdot \mathbf{1}^{\mathrm{T}}\right\}
\end{aligned}
$$

where $\rho=\left(\right.$ params, $\left.[\mathbf{p}]_{g},[\mathbf{w}]_{h}\right) \leftarrow \operatorname{Setup}\left(1^{\kappa}\right)$. Notice that the set of all the possible updates of a secret key, i.e.

$$
\left\{\phi: \phi(\cdot)=\operatorname{UpdateS}(\rho, \cdot ; \mathbf{A}), \mathbf{A} \in \mathbb{Z}_{q}^{n \times n}, \mathbf{1}_{n}=\mathbf{A} \cdot \mathbf{1}_{n}^{\mathrm{T}}, \operatorname{rank}(\mathbf{A})=d\right\},
$$

is a subset of $\Phi_{\rho}^{1}$. We show that $\left(\mathcal{R}_{\rho}^{1}, \Phi_{\rho}^{1}\right)$ is label-malleable friendly.

Representable statements and labels: Notice that $\mathcal{L}_{\mathcal{R}_{\rho}^{1}} \subseteq \mathbb{G}_{1}$, while the set of valid labels is the equal to $\mathbb{G}_{2}^{n \times m}$.

Representable transformations: We can describe a transformation $\phi_{\mathbf{A}} \in \Phi_{\rho}^{1}$ as a matrix of elements $[\mathbf{A}]_{h} \in \mathbb{G}_{2}^{n \times n}$.

Provable statements: The relation $\mathcal{R}_{\rho}^{1}$ can, obviously, be represented by the pairing product equation $[\alpha]_{g}=[m \cdot \mathbf{x}+\psi(\mathbf{r})]_{g}$.

Provable transformations: Given a transformation $\phi_{\mathbf{A}} \in \Phi_{\rho}^{1}$, and labels $s k=[\mathbf{S}]_{h}, s k^{\prime}=$ $\left[\mathbf{S}^{\prime}\right]_{h}$, the statement " $\phi_{\mathbf{A}}\left(s k^{\prime}\right)=s k \wedge \phi_{\mathbf{A}} \in \Phi^{1}$ " can expressed as a system of pairing product equations:

$$
\left\{\begin{array}{l}
{[\mathbf{A}]_{g} \bullet\left[\mathbf{S}^{\prime \mathrm{T}}\right]_{g}=[\mathbf{S}]_{g} \bullet[1]_{h}} \\
{[\mathbf{A}]_{g} \bullet\left[\mathbf{1}^{\mathrm{T}}\right]_{h}=[\mathbf{1}]_{f}}
\end{array}\right.
$$

Transformable transformations: Let $\phi_{\mathbf{A}}, s k, s k^{\prime}$ be as before, and let $\phi_{\mathbf{A}^{\prime}} \in \Phi_{\rho}^{1}$. We show that we can transform the system in Eq. (9) into a system of pairing product equations for the statement $\left(\phi_{\mathbf{A}^{\prime}} \circ \phi_{\mathbf{A}}\right)\left(s k^{\prime}\right)=\phi_{\mathbf{A}^{\prime}}(c) \wedge\left(\phi_{\mathbf{A}^{\prime}} \circ \phi_{\mathbf{A}}\right) \in \Phi$. In particular, we obtain

$$
\left\{\begin{array}{l}
{\left[\mathbf{A}^{\prime} \cdot \mathbf{A}\right]_{g} \bullet\left[\mathbf{S}^{\prime \mathrm{T}}\right]_{h}=\left[\mathbf{A}^{\prime} \cdot \mathbf{S}^{\mathrm{T}}\right]_{g} \bullet[1]_{h}} \\
{\left[\mathbf{A}^{\prime} \cdot \mathbf{A}\right]_{g} \bullet\left[\mathbf{1}^{\mathrm{T}}\right]_{h}=[\mathbf{1}]_{f}}
\end{array}\right.
$$

\section{Applications}

\subsection{Tamper-Resilient Signatures}

Consider a signature scheme $\mathcal{S S}$ (cf. Section 2.5). We would like to protect $\mathcal{S S}$ against tampering attacks with the memory, storing the signing key. As observed originally by Gennaro et al. [41], however, without further assumptions, this goal is too ambitious. Their attack can be circumvented by either assuming the self-destruct capability, or a key-update mechanism. 
Interestingly, Fujisaki and Xagawa [40] observed that, whenever the key-update mechanism is invoked only after an invalid output is generated, the goal of constructing tamper-resilient signature is impossible, even assuming the self-destruct capability. The idea behind the attack is to generate two valid pairs of independent signing/verification keys, and thus to overwrite the original secret key with either of the two sampled signing keys in order to signal one bit of the original key. Note that such an attack never generates invalid signatures, thus rendering both the self-destruct capability and a key-update mechanism useless.

\subsubsection{Split-State Signature Schemes}

A split-state signature scheme is a tuple of algorithms $\mathcal{S S}=$ (Setup, Gen, Sign, Vrfy, Rfrsh) specified as follows. (1) The (randomized) algorithm Setup takes as input the security parameter $\kappa \in \mathbb{N}$, and outputs public parameters $\rho \in\{0,1\}^{*}$; all algorithms are implicitly given $\rho$ as input. (2) The (randomized) algorithm Gen takes as input the public parameters $\rho \in\{0,1\}^{*}$, and outputs a pair of verification/signing key $(v k, s k)$, such that $s k$ consists of two shares $\left(S_{0}, S_{1}\right)$. (3) The (randomized) algorithm Sign takes as input two shares $S_{0}, S_{1}$, and a message $m \in\{0,1\}^{*}$, and outputs a signature $\sigma$. (4) The (deterministic) algorithm Vrfy takes as input the verification key $v k$, and a message/signature pair $(m, \sigma)$, and outputs a decision bit. (5) The (randomized) algorithm Rfrsh takes as input two shares $S_{0}, S_{1}$, and returns two updated shares $S_{0}^{\prime}, S_{1}^{\prime}$.

We say that $\mathcal{S} \mathcal{S}$ satisfies completeness if for all $\rho$ output by $\operatorname{Setup}\left(1^{\kappa}\right)$, for all $\left(v k,\left(S_{0}, S_{1}\right)\right)$ output by $\operatorname{Gen}(\rho)$, and for all messages $m \in\{0,1\}^{*}$, the following holds:

$$
\mathbb{P}\left[\operatorname{Vrfy}\left(v k, \operatorname{Sign}\left(s k, \operatorname{Rfrsh}\left(S_{0}, S_{1}\right), m\right)\right)\right] \geq 1-\nu(\kappa),
$$

for a negligible function $\nu$.

Informally, a split-state signature scheme is secure against continuous leakage and tampering attacks if no efficient adversary can forge a signature on a "fresh" message, even if it is given access to the following oracles: (i) A leakage oracle that returns entropy-bounded leakage on the shared secret key (computed on the two shares of the key independently); (ii) A tampering oracle that returns signatures on adaptively chosen messages, computed with a related secret key (obtained by modifying the two shares of the key independently). A formal definition (inspired by the work of Halevi and Lin [44] on after-the-fact leakage in PKE) follows below.

Definition 15 (Tamper-resilient signatures). Let $\mathcal{S S}=$ (Setup, Gen, Sign, Vrfy) be a split-state signature scheme. For any $\ell \in \mathbb{N}$, we say that $\mathcal{S S}$ is $\ell$-secure against continuous leakage and tampering attacks, if for all PPT $\ell$-valid adversaries $\mathrm{A}$ there is a negligible function $\nu: \mathbb{N} \rightarrow[0,1]$ such that

$$
\mathbb{P}\left[\operatorname{Exp}_{\mathcal{S} \mathcal{S}, A}^{\text {clt-cma }}(\kappa, \ell)=1\right] \leq \nu(\kappa),
$$

where the experiment is defined in Fig. 7.

\subsubsection{Construction and Analysis}

Let $\mathcal{S S}=($ Gen, Sign, Vrfy) be a signature scheme, and $\mathcal{C S}=$ (Init, Encode, Decode) be a coding scheme. Consider the following construction of a split-state signature scheme $\mathcal{S S}^{*}=$ (Setup*, Gen*, Sign*, Vrfy*, Rfrsh*).

- Setup* $\left(1^{\kappa}\right): \operatorname{Run} \omega \leftarrow \operatorname{Init}\left(1^{\kappa}\right)$ and output $\rho:=\omega$.

- Gen* $(\rho)$ : Parse $\rho:=\omega$, run $(p k, s k) \leftarrow \operatorname{Gen}\left(1^{\kappa}\right)$ and $\left(C_{0}, C_{1}\right) \leftarrow \operatorname{Encode}(\omega, s k)$; return $v k$ and $\left(S_{0}, S_{1}\right):=\left(C_{0}, C_{1}\right)$. 


$$
\begin{aligned}
& \text { Experiment } \operatorname{Exp}_{\mathcal{S S}, \mathrm{A}}^{\text {clt-cma }}(\kappa, \ell): \\
& \rho \leftarrow \operatorname{Setup}\left(1^{\kappa}\right) ; \mathcal{Q}:=\emptyset \\
& \left(v k,\left(S_{0}, S_{1}\right) \leftarrow \leftarrow \operatorname{Gen}(\rho) ; S:=\left(S_{0}, S_{1}\right)\right. \\
& \left(m^{*}, \sigma^{*}\right) \leftarrow\left(\mathrm{A}(\rho, v k) \leftrightarrows \mathcal{O}_{\text {sign }}(S), \mathcal{O}_{\mathrm{Rfrsh}}(S), \mathcal{O}_{\infty}(S)\right)
\end{aligned}
$$

Return 1 iff:

(i) $m^{*} \notin \mathcal{Q}$

(ii) $\operatorname{Vrfy}\left(v k,\left(m^{*}, \sigma^{*}\right)\right)=1$

$$
\begin{aligned}
& \text { Oracle } \mathcal{O}_{\text {sign }}\left(S_{0}, S_{1}, m, f_{0}, f_{1}\right): \\
& \left(\widetilde{S}_{0}, \widetilde{S}_{1}\right)=\left(f_{0}\left(S_{0}\right), f_{1}\left(S_{1}\right)\right) \\
& \mathcal{Q} \leftarrow \mathcal{Q} \cup\{m\} \\
& \sigma \leftarrow \operatorname{Sign}\left(\left(\widetilde{S}_{0}, \widetilde{S}_{1}\right), m\right) \\
& \text { If } \sigma=\perp \\
& \quad \text { Return } \perp \\
& \quad\left(S_{0}^{\prime}, S_{1}^{\prime}\right) \leftarrow \operatorname{Rfrsh}\left(S_{0}, S_{1}\right) \\
& \quad\left(S_{0}, S_{1}\right):=\left(S_{0}^{\prime}, S_{1}^{\prime}\right) \\
& \text { Else } \\
& \quad \text { Return } \sigma
\end{aligned}
$$

$$
\begin{aligned}
& \text { Oracle } \mathcal{O}_{\text {Rfrsh }}\left(S_{0}, S_{1}\right): \\
& \left(S_{0}^{\prime}, S_{1}^{\prime}\right) \leftarrow \operatorname{Rfrsh}\left(S_{0}, S_{1}\right) \\
& \left(S_{0}, S_{1}\right):=\left(S_{0}^{\prime}, S_{1}^{\prime}\right)
\end{aligned}
$$

Figure 7: Experiment defining unforgeability of a split-state signature scheme against continuous leakage and tampering attacks.

- $\operatorname{Sign}^{*}\left(\left(S_{0}, S_{1}\right), m\right)$ : Parse $\left(S_{0}, S_{1}\right):=\left(C_{0}, C_{1}\right)$, and let $\widetilde{s k}=\operatorname{Decode}\left(\omega,\left(C_{0}, C_{1}\right)\right)$; if the result is $\perp$, set $\sigma:=\perp$. Else, let $\sigma \leftarrow \operatorname{Sign}(s k, m)$. Output $\sigma$.

- $\operatorname{Vrfy}^{*}(v k,(m, \sigma))$ : Output $\operatorname{Vrfy}(v k,(m, \sigma))$.

- Rfrsh* $\left(S_{0}, S_{1}\right)$ : Parse $\left(S_{0}, S_{1}\right):=\left(C_{0}, C_{1}\right)$ and return $\left(C_{0}^{\prime}, C_{1}^{\prime}\right) \leftarrow \operatorname{Rfrsh}\left(\omega, C_{0}, C_{1}\right)$.

We prove the following theorem.

Theorem 4. Assume that $\mathcal{S S}$ is a EUF-CMA signature scheme, and that $\mathcal{C S}$ is a refreshable continuously non-malleable and $\ell$-leakage-resilient split-state code, then the above defined splitstate signature scheme $\mathcal{S S}^{*}$ is $\ell$-secure against continuous leakage and tampering attacks.

In the proof we define an intermediate experiment where tampering and leakage queries are answered by running the simulator of the underlying R-CNMC. Whenever the simulator returns $\diamond$, we replace the output of the simulator with a signature computed using the original secret key. By the security of the R-CNMC, the above experiment is computationally indistinguishable from the real experiment. Next, we show that no PPT adversary can forge in the intermediate experiment. The proof is down to the unforgeability of the underlying signature scheme $\Pi$. The main observation is that tampering queries can now either be simulated using the simulator of the R-CNMC, or using the signing oracle (in case the simulator returns $\diamond$ ).

Proof sketch. Let $\mathbf{G}_{\mathrm{A}}^{1}(\kappa):=\operatorname{Exp}_{\mathcal{S} \mathcal{S}, \mathrm{A}}^{\text {clt-cma }}(\kappa, \ell)$ be the experiment defined in Fig. 7, and denote by $\mathrm{S}=\left(\mathrm{S}_{0}, \mathrm{~S}_{1}\right)$ the simulator for the non-malleable code. Consider the hybrid experiment $\mathbf{G}_{\mathrm{A}}^{2}(\kappa)$ where we make two changes: (i) The public parameters $\omega$ are computed using the simulator $\mathrm{S}_{0}\left(1^{\kappa}\right)$; (ii) The signature oracle $\mathcal{O}_{\text {sign }}\left(S_{0}, S_{1}\right)$ is modified in such a way that, instead of running Decode as in the original experiment, we first run the simulator $S_{1}$ upon input $\left(f_{0}, f_{1}\right)$ in order to obtain a value $\tilde{s k} \in \mathcal{S K} \cup\{\diamond\}$, and then we proceed as before unless $\tilde{s k}=\diamond$, in which case the query is answered with $\sigma \leftarrow \operatorname{Sign}(s k, m)$.

Lemma 19. For all PPT adversaries $\mathrm{A}$, there is a negligible function $\nu_{1,2}: \mathbb{N} \rightarrow[0,1]$ such that

$$
\left|\mathbb{P}\left[\mathbf{G}_{\mathbf{A}}^{1}(\kappa)=1\right]-\mathbb{P}\left[\mathbf{G}_{\mathbf{A}}^{2}(\kappa)=1\right]\right| \leq \nu_{1,2}(\kappa) .
$$


Proof. Follows by a straightforward reduction to the non-malleability of the underlying code, as the only difference between the two games is that in the former game the tampering oracle always runs Sign on the output of Decode $\left(\omega, f_{0}\left(C_{0}\right), f_{1}\left(C_{1}\right)\right)$, whereas in the latter game we replaced the output of the decoding algorithm with the output of the simulator of the underlying code.

Lemma 20. For all PPT adversaries $\mathrm{A}$, there is a negligible function $\nu_{2}: \mathbb{N} \rightarrow[0,1]$ such that

$$
\mathbb{P}\left[\mathbf{G}_{\mathrm{A}}^{2}(\kappa)=1\right] \leq \nu_{2}(\kappa) .
$$

Proof. Follows by a straightforward reduction to the unforgeability of the underlying signature scheme, since the reduction can simulate signature queries on which the non-malleable code simulator returns $\diamond$ by using the target signing oracle (in the definition of EUF-CMA), whereas all other queries are already answered by running Sign on the output of the simulator of the underlying code.

\subsection{Tamper-Resilient RAM}

A read-only RAM program $\Lambda=(\Pi, \mathcal{D})$ consists of a next instruction function $\Pi$, a state state stored in a non-tamperable but non-persistent register, and some database $\mathcal{D}$. The next instruction function $\Pi$ takes as input the current state state and input inp, and outputs an instruction $I$ and a new state state'. The initial state is set to (start, $\star$ ).

A RAM compiler is a tuple of algorithms $\Sigma=$ (Setup, CompMem, CompNext) specified as follows. (1) Algorithm Setup takes as input the security parameter $1^{\kappa}$, and outputs an untamperable CRS $\omega$; (2) The memory compiler CompMem takes as input the CRS $\omega$, and a database $\mathcal{D}$, and outputs a database $\widehat{\mathcal{D}}$ along with an initial internal state state; (3) The next instruction function $\Pi$ is compiled to $\widehat{\Pi}$ using CompNext and the CRS. To define security, we compare two experiments (cf. Fig. 8). The real experiment features an adversary A that is allowed, via the interface doNext, to execute RAM programs on chosen inputs step-by-step; upon input $x$, oracle doNext $(x)$ outputs the result of a single step of the computation, as well as the memory location that is accessed during that step. Additionally, adversary A can also apply tampering attacks that are parameterized by two families of functions $\mathcal{F}_{\text {mem }}$ and $\mathcal{F}_{\text {bus }}$, where each function $f \in \mathcal{F}_{\text {mem }}$ is applied to the compiled memory, whilst each function $f \in \mathcal{F}_{\text {bus }}$ is applied to the data in transit on the bus.

The ideal experiment features a simulator $\mathbf{S}$ that is allowed, via the interface Execute, to execute RAM programs on chosen inputs. Upon input $x$, oracle Execute $(x)$ outputs the result of the entire computation and the list of all the memory locations that were accessed during that computation. Briefly, a RAM compiler is tamper-resilient if for all possible logics $\Pi$, and all efficient adversaries $\mathrm{A}$, there exists a simulator $\mathrm{S}$ such that the real and ideal experiment are computationally indistinguishable. A formal definition follows.

Definition 16 (Tamper simulatability). A compiler $\Sigma=$ (Setup, CompMem, CompNext) is tamper simulatable w.r.t. $\left(\mathcal{F}_{\text {bus }}, \mathcal{F}_{\text {mem }}\right)$ if for every next instruction function $\Pi$, and for every PPT adversary $\mathrm{A}$, there exists a PPT simulator $\mathrm{S}$ and a negligible function $\nu: \mathbb{N} \rightarrow[0,1]$ such that, for all PPT distinguisher $\mathrm{D}$ and any database $\mathcal{D}$, we have that:

$$
\left|\mathbb{P}\left[\mathrm{D}\left(\operatorname{TamperExec}_{\mathrm{A}, \Sigma, \Lambda}^{\mathcal{F}_{\text {bus }}, \mathcal{F}_{\text {mem }}}(\kappa)\right)=1\right]-\mathbb{P}\left[\mathrm{D}\left(\operatorname{IdealExec}_{\mathrm{S}, \Lambda}(\kappa)\right)=1\right]\right| \leq \operatorname{negl}(\kappa)
$$

with $\Lambda:=(\Pi, \mathcal{D})$, and where the experiments $\operatorname{TamperExec}_{\mathrm{A}, \Sigma, \Lambda}^{\mathcal{F}_{\text {bus }}, \mathcal{F}_{\text {mem }}}$ and $\operatorname{IdealExec}_{\mathrm{S}, \Lambda}(\kappa)$ are defined in Fig. 8. 
Experiment TamperExec $\operatorname{Ex}_{\mathrm{A}, \Sigma, \Lambda}^{\mathcal{F}_{\text {bus }}, \mathcal{F}_{\text {mem }}}(k):$

$\omega \leftarrow \operatorname{Setup}\left(1^{\kappa}\right)$

Parse $\Lambda$ as $(\overline{\mathcal{D}}, \bar{\Pi}) ; \mathcal{Q} \leftarrow \emptyset$;

$\mathcal{D} \leftarrow \operatorname{CompMem}(\omega, \overline{\mathcal{D}}), \mathcal{D}^{\prime} \leftarrow \mathcal{D} ;$

$\Pi \leftarrow \operatorname{CompNext}(\omega, \bar{\Pi})$;

$b \leftarrow\left(\mathrm{A}(\omega) \leftrightarrows \operatorname{doNext}\left(\left(\mathcal{D}^{\prime}, \Pi\right), \cdot\right), \mathcal{O}_{\text {tamp }}(\cdot)\right) ;$

Return $(b, \mathcal{Q})$.

Experiment IdealExec $_{S, \Lambda}(\kappa)$ :

$\overline{\mathcal{Q} \leftarrow \emptyset ;}$

$b \leftarrow\left(\mathrm{S}\left(1^{\kappa}\right) \leftrightarrows \operatorname{Execute}(\Lambda, \cdot), \operatorname{Add}(\cdot)\right)$

Return $(b, \mathcal{Q})$.

Oracle $\operatorname{Add}(x)$ :

$\overline{\mathcal{Q}} \leftarrow \mathcal{Q} \cup\{x\} ;$

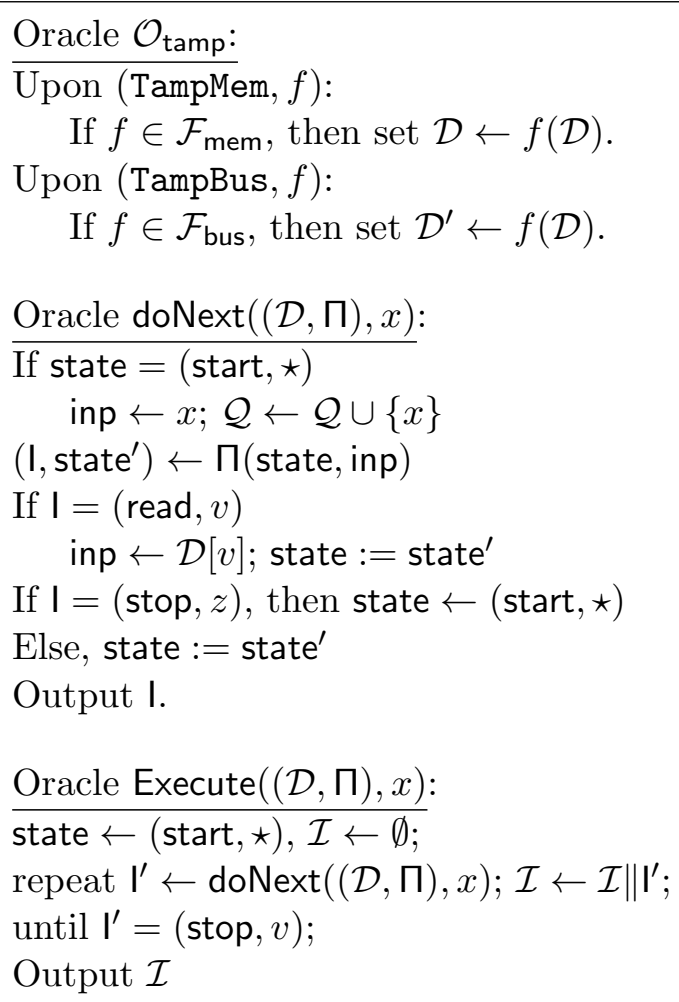

Figure 8: Experiments defining security of a RAM compiler.

\subsubsection{Tampering on the Bus}

Roughly, our RAM compiler encodes a randomly generated secret key $k$ of an authenticated encryption scheme using a R-CNMC, creating a codeword $\left(K_{0}, K_{1}\right)$. Next, it encrypts each data block in the memory under the key $k$. Let $\mathcal{E}$ be the encrypted blocks. The compiled database consists of two parts $\left(K_{0}, \mathcal{E}\right)$ and $\left(K_{1}, \mathcal{E}\right)$. A fundamental twist w.r.t. to previous work is that we include the encrypted database in both the parts. This feature allows us to prove security in the split-state model, at the price of requiring the following additional property for the underlying R-CNMC.

Definition 17 (Codeword correlation). A split-state code $\mathcal{C S}=$ (Init, Encode, Decode) has $\alpha$ correlated codewords if for all $\omega$ output by Init, any message $M \in \mathcal{M}$, and $\left(C_{0}, C_{1}\right) \leftarrow \operatorname{Enc}(\omega, M)$, the following holds for all $\beta \in\{0,1\}: \widetilde{\mathbb{H}}_{\infty}\left(C_{\beta} \mid C_{1-\beta}\right) \geq \mathbb{H}_{\infty}\left(C_{\beta}\right)-\alpha$.

It is easy to verify that the code from Section 4 has $\alpha$-correlated codewords for $\alpha:=$ $\max \{\delta(\kappa), \mu(\kappa)\} \quad($ where $\delta:=\log |\mathcal{M}|$ and $\delta:=\log |\mathcal{P K}|)$.

Let $\mathcal{S} \mathcal{K} \mathcal{E}=($ KGen, Enc, Dec) be a secret-key encryption scheme. Let $\mathcal{C S}=($ Init, Encode, Decode) be a R-CNMC. Our tamper-resilient read-only RAM compiler $\Sigma=$ (Setup, CompMem, CompNext) works as follows.

- $\operatorname{Setup}\left(1^{\kappa}\right): \operatorname{Run} \omega \leftarrow \operatorname{Init}\left(1^{\kappa}\right)$ and output $\omega$.

- CompMem $(\omega, \mathcal{D})$ : Run $k \leftarrow \operatorname{KGen}\left(1^{\kappa}\right)$ and $\left(K_{0}, K_{1}\right) \leftarrow \operatorname{Encode}(\omega, k)$. For each $d_{i}$ in $\mathcal{D}=$ $\overline{\left(d_{1}, \ldots, d_{n}\right) \text { do } c_{i}} \leftarrow \operatorname{Enc}\left(k, d_{i} \| i\right)$ and let $\mathcal{E}=\left(c_{1}, \ldots, c_{n}\right)$. Output $\left(\mathcal{D}_{0}, \mathcal{D}_{1}\right)$, where $\mathcal{D}_{0}=$ $\left(K_{0}, \mathcal{E}\right)$ and $\left.\mathcal{D}_{1}=\left(K_{1}, \mathcal{E}\right)\right)$, and let the initial internal state be state $=\left((\right.$ start, $\left.\star)\left\|0^{\kappa}\right\| 0^{\log n}\right)$.

- CompNext $(\omega, \Pi)$ : The compiled next instruction function $\widehat{\Pi}$ does the following: Upon input (state, inp $),$ parse state $=\left(\right.$ state $\left._{\text {RAm }}\left\|k_{\text {last }}\right\| I_{\text {last }}\right)$. Hence: 
- If state RAM $=($ start,$\star)$

1. $\left(\right.$ I, state RAM $\left._{\text {RA }}^{\prime}\right) \leftarrow \Pi\left(\right.$ state $_{\text {RAM }}$, inp $)$;

2. Read from $\mathcal{D}_{0}$ the value $K_{0}$ and from $\mathcal{D}_{1}$ the value $K_{1}$;

3. Compute $k \leftarrow \operatorname{Decode}\left(\omega,\left(K_{0}, K_{1}\right)\right)$;

4. Set state $\leftarrow\left(\right.$ state $\left._{\text {RAM }}^{\prime}\|k\| I\right)$;

5. Output (I, state).

- If $\operatorname{state}_{\text {RAM }} \neq($ start,$\star)$

1. Parse $\mathbf{I}_{\text {last }}$ as $($ read, $v$ );

2. Read $c_{v}$ from $\mathcal{D}_{0}$ and read $c_{v}^{\prime}$ from $\mathcal{D}_{1}$;

3. Compute $d^{\prime} \| i^{\prime} \leftarrow \operatorname{Dec}\left(k_{\text {last }}, c_{v}\right)$;

4. If $\left(c_{v} \neq c_{v}^{\prime}\right)$ or $d^{\prime} \| i^{\prime}=\perp$ or $i^{\prime} \neq v$, then signal $\mathcal{D}_{0}$ and $\mathcal{D}_{1}$ to refresh, set state $\leftarrow\left((\right.$ start, $\left.\star)\left\|0^{\kappa}\right\| 0^{\log n}\right)$ and output $\perp$.

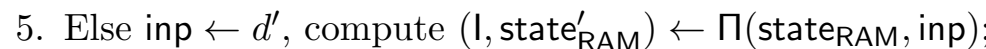

6. If $\mathrm{I}=($ stop, $z)$, then set state $\leftarrow\left((\right.$ start, $\left.\star)\left\|0^{\kappa}\right\| 0^{\log n}\right)$ and output (I, state);

7. Else set state $\leftarrow$ (state RAM $\left.^{\prime}\left\|k_{\text {last }}\right\| \mathrm{I}\right)$ and output (I, state).

Theorem 5. Assume that $\mathcal{S K E}$ is an authenticated encryption scheme with ciphertexts size bounded by $q(\kappa, n) \in \operatorname{poly}(\kappa)$, and that $\mathcal{C S}$ is a $(q+\alpha+O(\log \kappa))$-leakage-resilient and continuously non-malleable code with split-state refresh, that additionally has $\alpha$-correlated codewords. Then, the above construction defines a tamper-resilient $R A M$ compiler w.r.t. $\left(\mathcal{F}_{\text {bus }}, \emptyset\right)$, where $\mathcal{F}_{\text {bus }}$ is the class of split-state tampering functions applied on the bus.

Proof. Let $\mathcal{C}_{E} \subset\{0,1\}^{q(\kappa, n)}$ and $\mathcal{C}^{0} \times \mathcal{C}^{1}$ be, respectively, the ciphertext space of $\mathcal{S} \mathcal{K} \mathcal{E}$ and the codeword space of $\mathcal{C O M}$. Without loss of generality, we can parse a function $g \in \mathcal{F}_{\text {bus }}$ as $\left(\left.g\right|_{\text {key }},\left.g\right|_{\text {mem }}\right)$ where, in turn, $\left.g\right|_{\text {key }}:=\left(T^{0}, T^{1}\right)$ and $\left.g\right|_{\text {mem }}:=\left(M^{0}, M^{1}\right)$. Here:

- The function $T^{0}$ has domain $\mathcal{C}^{0} \times \mathcal{C}_{E}^{n}$ and co-domain $\{0,1\}^{p(\kappa)}$;

- The function $T^{1}$ has domain $\mathcal{C}^{1} \times \mathcal{C}_{E}^{n}$ and co-domain $\{0,1\}^{p(\kappa)}$;

- The function $M^{0}$ has domain $\mathcal{C}^{0} \times \mathcal{C}_{E}^{n}$ and co-domain $\{0,1\}^{q(\kappa, n)}$.

- The function $M^{1}$ has domain $\mathcal{C}^{1} \times \mathcal{C}_{E}^{n}$ and co-domain $\{0,1\}^{q(\kappa, n)}$.

Informally, $\left.g\right|_{\text {key }}$ acts over the codeword of the NMC and $\left.g\right|_{\text {mem }}$ acts over the $n$ ciphertexts. To prove security of our construction we need to construct a simulator S. Let $L_{\mathrm{cptx}}^{i, j}$ be the leakage function that upon input $K_{j}$ first computes $\tilde{\mathcal{E}} \leftarrow M^{j}\left(K_{j}, \mathcal{E}\right)$, and then outputs $\tilde{\mathcal{E}}[i]$. Namely, $L_{\mathrm{cptx}}^{i, j}$ is the function that leaks the $i$-th ciphertext from the tampered database. Let $\left(\mathrm{S}_{0}, \mathrm{~S}_{1}\right)$ denote the simulator for $\mathcal{C S}$.

Simulator S:

1. Generate $\omega \leftarrow \mathrm{S}_{0}\left(1^{\kappa}\right)$, let $k \leftarrow \mathrm{KGen}\left(1^{\kappa}\right)$ a key for the authenticated encryption, create an encrypted dummy database $\mathcal{D}$, where each entry is an encryption of $0^{\kappa}$, i.e. $\mathcal{D}=$ $\left(c_{1}, \ldots, c_{n}\right)$ where $c_{i}=\operatorname{Enc}\left(k, 0^{\kappa} \| i\right)$. Run the simulator $\mathrm{S}_{1}$. Run the real world adversary A with input $\omega$. Let $f l g_{\text {key }}$ be the flag that indicates the current state of the key. Initially the flag is set to OK. Let $f \lg _{\text {start }}$ be the flag that indicates the current state of the execution. Initially the flag is set to False. 
2. Upon receiving a query from the adversary A proceed as follows:

If the query is for $\mathcal{O}_{\text {tamp }}$ and it is of the kind (TampBus, $g$ ) and $g \in \mathcal{F}_{\text {bus }}$ then set $g$ as the current tampering function.

If the query is for doNext with input $x$, in case $f \lg _{\text {start }}=$ False then set $g^{\prime}:=\left(T^{0}\left((\cdot, \mathcal{D}), T^{1}(\cdot\right.\right.$, $\mathcal{D})$ ) where $\left(T^{0}, T^{1}\right)$ is $\left.g\right|_{\text {key }}$ and $g$ is the current tampering query, forward $g^{\prime}$ to $\mathrm{S}_{1}$, and do the following:

(a) If the simulator outputs $\diamond$, we set $f \lg _{\text {key }} \leftarrow$ OK.

(b) If the simulator outputs $\perp$, we set $f \lg _{\text {key }} \leftarrow$ broken.

(c) The last option for the simulator is to output a valid, but unrelated, key $k^{*}$; in this case, we set $f \lg _{\text {key }} \leftarrow$ unrelated.

Finally, set the flag $f \lg _{\text {start }}$ to True. Afterwards, do the following depending on $f l g_{\text {key }}$ :

(a') If $f \lg _{\text {key }}=$ broken, then send the query $x$ to $\operatorname{Add}(\cdot)$ and answer with $\perp$.

(b') If $f \lg _{\text {key }}=$ unrelated then if $\mathrm{cnt}=0$ send the query $x$ to $\operatorname{Add}(\cdot)$; compute locally:

- If state RAM $=($ start,$\star)$ set inp $\leftarrow x$ and execute $\left(\right.$ I, state RAM $\left._{\text {RA }}\right) \leftarrow \Pi$ (state RAM $\left._{\text {, inp }}\right)$, set state $\leftarrow\left(\operatorname{state}_{\mathrm{RAM}}^{\prime}\left\|k^{*}\right\| \mathrm{I}\right)$, increment cnt and return I;

- If $\mathbf{I}_{\text {last }}=(\mathrm{read}, v)$ send the leakage oracle queries (Leak, $\left.\left(0, L_{\mathrm{cptx}}^{v, 0}\right)\right)$ and (Leak, $(1$, $\left.L_{\text {cptx }}^{v, 1}\right)$ ) to the simulator $\mathrm{S}_{1}$. Let $\widetilde{c}_{v}$ and $\widetilde{c}_{v}$ be the respective answers. Decrypt $\left(d^{\prime} \| i^{\prime}\right) \leftarrow \operatorname{Dec}\left(k^{*}, \widetilde{c}_{v}\right)$. If $\widetilde{c}_{v} \neq \widetilde{c}_{v}$ or $\left(d^{\prime} \| i^{\prime}\right)=\perp$ or $i^{\prime} \neq v$ then output $\perp$. Otherwise, set inp $\leftarrow d^{\prime}$ and compute locally (I, state RAM $\left.^{\prime}\right) \leftarrow \Pi$ (state RAM $_{\text {, inp) set }}$ state $\leftarrow\left(\right.$ state $\left._{\text {RAM }}^{\prime}\left\|k^{*}\right\| I\right)$ increment cnt and return I.

- If $\mathbf{I}_{\text {last }}=($ stop, $v)$ output $\mathcal{I}$, set state $\leftarrow\left((\right.$ start, $\left.\star),\left\|0^{\kappa}\right\| 0^{\log n}\right)$ and reset cnt to 0 and return $\mathrm{I}$.

(c') If $f l g_{\text {key }}=$ OK then if $\mathrm{cnt}=0$ forward the query $x$ to $\operatorname{Execute}(\Lambda, \cdot)$, let $\mathcal{I}$ the answer, else if cnt $>0$ retrieve $\mathcal{I}$. Parse $\mathcal{I}=\left(\mathrm{I}_{1}, \ldots, \mathrm{I}_{p}\right)$ for $p \in \mathbb{N}$. If $\mathrm{I}_{\mathrm{cnt}}$ is of the form (read, $v$ ) then send the leakage oracle queries (Leak, $\left(0, L_{\text {cptx }}^{v, 0}\right)$ and (Leak, $\left(1, L_{\text {cptx }}^{v, 1}\right)$ ) to the simulator $S_{1}$. Let $\widetilde{c}_{v}$ and $\widetilde{c}_{v}$ be the respective answers. If $\widetilde{c}_{v}=\mathcal{E}[v]=\widetilde{c}_{v}$ then output $I_{\text {cnt }}$ and increment the counter else output $\perp$.

3. In all of the above cases, whenever return $\perp$ to the adversary, force ${ }^{13}$ the simulator $S_{1}$ to refresh and reset the flag $f \lg _{\text {key }}$ to OK and the flag $f \lg _{\text {start }}$ to False.

We prove the indistinguishability in 3 steps. Let $\mathrm{H}_{i}$ be the $i$-th hybrid adversary, such adversary takes as input $1^{\kappa}$ and the original database $\mathcal{D}$ and interacts with doNext. The latter defines the hybrid experiment $\mathbf{H}_{i}$, specifically $\mathbf{H}_{i}(\kappa):=\mathrm{D}\left(\mathrm{H}_{i}\left(1^{\kappa}, \mathcal{D}\right) \leftrightarrows \operatorname{Execute}(\Lambda, \cdot), \operatorname{Add}(\cdot)\right)$ where $\Lambda=(\Pi, \mathcal{D})$. Let the hybrid adversaries be defined as follows:

Hybrid adversary $\mathrm{H}_{1}$. It takes as input the database $\mathcal{D}$ and executes the same code of the simulator $\mathbf{S}$, but it honestly encrypts the database $\mathcal{D}$. Namely, $\mathcal{E}=\left(c_{1}, \ldots, c_{n}\right)$ where $c_{i} \leftarrow \operatorname{Enc}\left(k,\left(d_{i} \| i\right)\right)$ for all $1 \leq i \leq n$.

Hybrid adversary $\mathrm{H}_{2}$. It takes as input the database $\mathcal{D}$ and executes the same code of the hybrid adversary $\mathrm{H}_{1}$, but in point 2.(c') it does not check that $\widetilde{c}_{v}=\mathcal{E}[v]=\widetilde{c}_{v}$ (where

\footnotetext{
${ }^{13}$ This can be done by sending a tampering query that overwrites the target encoding with an invalid codeword, thus triggering a decoding error.
} 
$\widetilde{c}_{v}:=L_{\text {cptx }}^{v, 0}\left(K_{0}\right)$ and $\left.\widetilde{c}_{v}:=L_{\text {cptx }}^{v, 1}\left(K_{1}\right)\right)$. Instead it decrypts $\left(d^{\prime} \| i^{\prime}\right)=\operatorname{Dec}\left(k^{*}, \widetilde{c}_{v}\right)$ and if $\widetilde{c}_{v} \neq \widetilde{c}_{v}$ or $\left(d^{\prime} \| i^{\prime}\right)=\perp$ or $i^{\prime} \neq v$ then outputs $\perp$ and refreshes $K_{0}, K_{1}$. (Namely, it executes the same code the compiler CompNext would execute.)

Hybrid adversary $\mathrm{H}_{3}$. It takes as input the database $\mathcal{D}$ and executes the same code of the hybrid adversary $\mathrm{H}_{2}$, but instead of running the simulator $\mathrm{S}_{1}$ of the R-CNMC, it samples $\left(K_{0}, K_{1}\right) \leftarrow \operatorname{Encode}(\omega, k)$, computes the decoding, the leakage function $L_{\mathrm{cptx}}$ and the refreshing algorithm directly on $K_{0}, K_{1}$. In particular, whenever it computes a decoding it does the following checks. If the decoded key $k^{*}$ is $\perp$, then set the flag $f \lg _{\text {key }} \leftarrow$ broken, else if $k^{*}$ is equal to the original key $k$, then set $f \lg _{\text {key }} \leftarrow \mathrm{OK}$, and else set $\mathrm{fl} \mathrm{lg}_{\text {key }} \leftarrow$ unrelated.

Lemma 21. For any read-only $R A M \Lambda=(\Pi, \mathcal{D})$ there exists a negligible function $\nu: \mathbb{N} \rightarrow[0,1]$ such that $\mid \mathbb{P}\left[\mathbf{H}_{1}(\kappa)=1\right]-\mathbb{P}\left[\right.$ IdealExec $\left._{\mathrm{S}, \Lambda}(\kappa)=1\right] \mid \leq \nu(\kappa)$.

Proof sketch. We reduce to the security of the authenticated encryption via a hybrid argument. Let $\mathrm{H}_{1}^{i}$ be the hybrid adversary that executes the same code of $\mathrm{H}_{1}$ but encrypts to $\mathcal{D}$ only the first $i$ locations and the remaining are encrypted to 0 . We prove that for any index $i \in[n-1]$ there exists a negligible function $\nu^{\prime}$ such that

$$
\left|\mathbb{P}\left[\mathbf{H}_{1}^{i}\left(1^{\kappa}\right)=1\right]-\mathbb{P}\left[\mathbf{H}_{1}^{i+1}\left(1^{\kappa}\right)=1\right]\right| \leq \nu^{\prime}(\kappa),
$$

where $\mathbf{H}_{1}^{i}\left(1^{\kappa}\right):=\mathrm{D}\left(\mathrm{H}_{1}^{i}\left(1^{\kappa}, \mathcal{D}\right) \leftrightarrows \operatorname{Execute}(\Lambda, \cdot), \operatorname{Add}(\cdot)\right)$

Let $\mathrm{B}$ the adversary that upon input $1^{\kappa}$ and oracle access to $\operatorname{Dec}(k, \cdot)$ executes the same code of the hybrid adversary $\mathrm{H}_{1}^{i}$ but queries its own oracle for the encryption of $(\mathcal{D}[i] \| j)$ for $0 \leq j \leq i$. The adversary $\mathrm{B}$ outputs $(\mathcal{D}[i] \| i, 0)$ as challenge messages and gets $c$ as challenge ciphertext. It is easy to see that $B$ fully simulates $H_{1}^{i}$ if the challenge bit is 0 and $\mathrm{H}_{1}^{i+1}$ if the challenge bit is 1 which implies a distinguisher against the semantic security of $\mathcal{S K} \mathcal{E}$.

Lemma 22. For any read-only $R A M \Lambda=(\Pi, \mathcal{D})$ there exists a negligible function $\nu: \mathbb{N} \rightarrow[0,1]$ such that $\left|\mathbb{P}\left[\mathbf{H}_{1}(\kappa)=1\right]-\mathbb{P}\left[\mathbf{H}_{2}(\kappa)=1\right]\right| \leq \nu(\kappa)$.

Proof sketch. The lemma follows from the authenticity of $\mathcal{S} \mathcal{K} \mathcal{E}$. In particular, let Forge be the event that $\widetilde{c}_{v} \neq \mathcal{E}[v] \neq \widetilde{c}_{v}$ but $\widetilde{c}_{v}=\widetilde{c}_{v}$ and $\left(d^{\prime} \| i^{\prime}\right) \neq \perp$ and $i^{\prime}=v$ happens in $\mathbf{H}_{2}$. The event Forge allows to distinguish between $\mathbf{H}_{2}$ and $\mathbf{H}_{3}$, therefore $\left|\mathbb{P}\left[\mathbf{H}_{2}(\kappa)=1\right]-\mathbb{P}\left[\mathbf{H}_{3}(\kappa)=1\right]\right| \leq \mathbb{P}[$ Forge]. We prove that if $\mathbb{P}[$ Forge $] \geq 1 / p(\kappa)$ for a polynomial $p$, then we can break authenticity of $\mathcal{S} \mathcal{K} \mathcal{E}$ with the same probability. Notice that since $\widetilde{c}_{v} \neq \mathcal{E}[v]$ and $i^{\prime}=v$ then for any $i \in[n]$ we have that $\widetilde{c}_{v} \neq \mathcal{E}[v]$; this is because the other ciphertexts encrypt a different index. This means that $\widetilde{c}_{v}$ is fresh and moreover it decrypts correctly under the key $k$ (since $d^{\prime} \| i^{\prime} \neq \perp$ ). The reduction is straightforward and therefore the details are omitted.

Lemma 23. For any read-only $R A M \Lambda=(\Pi, \mathcal{D})$ there exists a negligible function $\nu: \mathbb{N} \rightarrow[0,1]$ such that $\left|\mathbb{P}\left[\mathbf{H}_{2}(\kappa)=1\right]-\mathbb{P}\left[\mathbf{H}_{3}(\kappa)=1\right]\right| \leq \nu(\kappa)$.

Proof sketch. The lemma follows from the security of the R-CNMC. There are two things to notice. First, the function $\left.g\right|_{\text {key }}$ for any assignment of the encrypted database $c_{1}, \ldots, c_{n}$ is a valid split-state tampering function. Second, by continuously tampering with the encrypted splitstate database, for each round, the adversary can leak information on $K_{0}, K_{1}$. In particular, both hybrid adversaries compute a sequence of leakage functions on $K_{0}, K_{1}$ from the set $\mathcal{L}$ defined ${ }^{14}$ below:

$$
\mathcal{L}:=\left\{\left(L_{\text {cptx }}^{i, 0}, L_{\text {cptx }}^{i, 1}\right): i \in \mathbb{N}\right\}
$$

\footnotetext{
${ }^{14}$ Notice that the leakage functions depends on $\left.g\right|_{\text {mem }}$ which can change during the execution of one round. For simplicity we hide this extra parameter.
} 
We show that, for every round, the sequence of leakage functions applied during that round is below the leakage bound of $\mathcal{C S}$. Fix a round and let $\bar{L}:=\left(\left(L_{1}^{0}, L_{1}^{1}\right),\left(L_{2}^{0}, L_{2}^{1}\right), \ldots,\left(L_{p}^{0}, L_{p}^{1}\right)\right)$ be such a sequence, where $p(\kappa) \in \operatorname{poly}(\kappa)$ is a polynomial.

$$
\begin{aligned}
\widetilde{\mathbb{H}}_{\infty}\left(K_{j} \mid \bar{L}\left(K_{0}, K_{1}\right)\right) \\
\quad \geq \widetilde{\mathbb{H}}_{\infty}\left(K_{j} \mid L_{1}^{j}\left(K_{j}\right), \ldots, L_{p}^{j}\left(K_{j}\right), K_{1-j}\right) \\
\quad \geq \widetilde{\mathbb{H}}_{\infty}\left(K_{j} \mid L_{1}^{1-j}\left(K_{1-j}\right), \ldots, L_{p-1}^{1-j}\left(K_{1-j}\right), L_{p}^{j}\left(K_{j}\right), K_{1-j}\right) \\
\quad \geq \widetilde{\mathbb{H}}_{\infty}\left(K_{j} \mid L_{1}^{1-j}\left(K_{1-j}\right), \ldots, L_{p-1}^{1-j}\left(K_{1-j}\right), L_{p}^{j}\left(K_{j}\right), K_{1-j}\right) \\
\quad \geq \widetilde{\mathbb{H}}_{\infty}\left(K_{j} \mid p, L_{p}^{j}\left(K_{j}\right), K_{1-j}\right) \\
\quad \geq \mathbb{H}_{\infty}\left(K_{j}\right)-O(\log \kappa)-q(\kappa, n)-\alpha(\kappa) .
\end{aligned}
$$

Where the first equation follows by the fact that, for any $i$, the value $L_{i}^{1-j}\left(K_{1-j}\right)$ is a function of $K_{1-j}$, the second equation follows by the fact that for any $i<p$, by definition of $\mathrm{H}_{1}$ and $\mathrm{H}_{2}$, $L_{i}^{1-j}\left(K_{1-j}\right)=L_{i}^{j}\left(K_{j}\right)$ holds, and in the forth equation we applied the $\alpha$-correlated-codeword property of $\mathcal{C S}$ and the bound on the size of a ciphertext for $\mathcal{S} \mathcal{K} \mathcal{E}$.

Lemma 24. $\mathbb{P}\left[\mathbf{H}_{3}(\kappa)=1\right]=\mathbb{P}\left[\mathrm{D}\left(\operatorname{TamperExec}_{\mathrm{A}, \Sigma, \Lambda}^{\mathcal{F}_{\text {bus }}, \emptyset}(\kappa)\right)=1\right]$.

Proof sketch. For each query to doNext with input $x$, the hybrid experiment checks the flag $\mathrm{flg}_{\text {key }}$. In particular:

- If the flag is set to broken, then it means that Decode $\left(\widetilde{K}_{0}, \widetilde{K}_{1}\right)$ is $\perp$ (by inspection), therefore in this case the real experiment would run, add $x$ to the set of queries and output $\perp$; the hybrid $\mathbf{H}_{3}$ does the same using its interface Add.

- If the flag is set to unrelated, then it means that Decode $\left(\widetilde{K}_{0}, \widetilde{K}_{1}\right)=k^{*} \neq k$ (by inspection). In this case the hybrid experiment simulates perfectly the real experiment, by computing locally as would happen in the real experiment, moreover the hybrid add the query to $\mathcal{Q}$ using its interface Add.

- If the flag is set to OK, then it means that Decode $\left(\widetilde{K}_{0}, \widetilde{K}_{1}\right)=k$. In this case the hybrid experiment uses its interface Execute, but before it outputs the transcript it processes it by finding the point where the real experiment would output $\perp$ (if the latter, indeed, outputs $\perp$ ).

\subsubsection{Tampering on both the Bus and the Memory}

The first compiler is not secure against adversaries that can tamper persistently ${ }^{15}$ with the memory (in the split-state model) if we do not assume self-destruct. The reason is that only part of the memory is refreshed: the adversary can use the remaining part to backup the old encoding and then rewind the refreshing procedure. (See also the discussion in Section 1.3.) To partially overcome this problem we assume that, once a decoding error is triggered, the system can switch into a safe mode where the communication between the CPU and the memory is tamper free. While in safe mode, the system will perform a consistency check. To minimize the

\footnotetext{
${ }^{15}$ Note that tampering on the bus constitutes a form of non-persistent tampering.
} 
dependency on the assumption we constraint the consistency check to be succinct, meaning that its complexity depends only on the security parameter and not on the size of the RAM program. Finally, if the consistency check passes, the refresh procedure will be executed otherwise a selfdestruct is triggered.

The compiler. Let $\mathcal{S K E}=($ KGen, Enc, Dec) be a secret-key encryption scheme. Let $\mathcal{C S}=$ (Init, Encode, Decode) be a R-CNMC. Our tamper-resilient read-only RAM compiler $\Sigma=$ (Setup, CompMem, CompNext) works as follows.

- $\operatorname{Setup}\left(1^{\kappa}\right): \operatorname{Run} \omega \leftarrow \operatorname{Init}\left(1^{\kappa}\right)$ and output $\omega$.

- CompMem $(\omega, \mathcal{D})$ : Run $k \leftarrow \operatorname{KGen}\left(1^{\kappa}\right)$ and $\left(K_{0}, K_{1}\right) \leftarrow \operatorname{Encode}(\omega, k)$. For each $d_{i}$ in

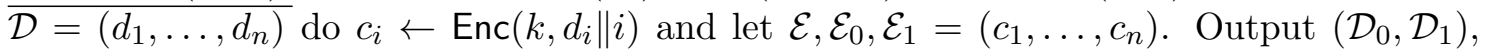
where $\mathcal{D}_{0}=\left(K_{0}, \mathcal{E}_{0}\right)$ and $\left.\mathcal{D}_{1}=\left(K_{1}, \mathcal{E}_{1}\right)\right)$, and let the initial internal state be state $=$ $\left((\right.$ start,$\left.\star)\left\|0^{\kappa}\right\| 0^{\log n}\right)$.

- CompNext $(\omega, \Pi)$ : The compiled next instruction function $\widehat{\Pi}$ does the following. Upon input (state, inp $)$, parse state $=\left(\right.$ state $\left._{R A M}\left\|k_{\text {last }}\right\| \mathbf{l}_{\text {last }}\right)$.

- If state RAM $=($ start,$\star)$

1. (I, state $\left._{R A M}^{\prime}\right) \leftarrow \Pi\left(\right.$ state $_{R A M}$, inp $)$;

2. Read from $\mathcal{D}_{0}$ the value $K_{0}$ and from $\mathcal{D}_{1}$ the value $K_{1}$;

3. Compute $k \leftarrow \operatorname{Decode}\left(\omega,\left(K_{0}, K_{1}\right)\right)$;

4. If $k=\perp$ switch to safe mode and perform the consistency check:

(a) Retrieve $K_{0}, K_{1}$ from memory and if $\operatorname{Decode}\left(\omega, K_{0}, K_{1}\right)=\perp$ then selfdestruct;

(b) Sample $i_{1}, \ldots, i_{\zeta} \leftarrow[n]$ and for $l \in[\zeta]$ check if $\mathcal{E}_{0}\left[i_{l}\right] \neq \mathcal{E}_{1}\left[i_{l}\right]$ then selfdestruct else continue.

Switch back to normal mode, refresh, and signal $\mathcal{D}_{0}$ and $\mathcal{D}_{1}$ to refresh. Set state $\leftarrow\left((\right.$ start,$\left.\star)\left\|0^{\kappa}\right\| 0^{\log n}\right)$ and output $\perp$.

5. Else set state $\leftarrow\left(\right.$ state $\left._{R A M}^{\prime}\|k\| I\right)$;

6. Output (I, state).

- If state $_{R A M} \neq($ start,$\star)$

1. Parse $\mathrm{I}_{\text {last }}$ as $(\mathrm{read}, v)$;

2. Read $c_{v}$ from $\mathcal{D}_{0}$ and read $c_{v}^{\prime}$ from $\mathcal{D}_{1}$;

3. Compute $d^{\prime} \| i^{\prime} \leftarrow \operatorname{Dec}\left(k_{\text {last }}, c_{v}\right)$;

4. If $\left(c_{v} \neq c_{v}^{\prime}\right)$ or $d^{\prime} \| i^{\prime}=\perp$ or $i^{\prime} \neq v$, then switch to safe mode, perform the consistency check, switch back to normal mode and signal $\mathcal{D}_{0}$ and $\mathcal{D}_{1}$ to refresh, set state $\leftarrow\left((\right.$ start,$\left.\star)\left\|0^{\kappa}\right\| 0^{\log n}\right)$ and output $\perp$.

5. Else inp $\leftarrow d^{\prime}$, compute $\left(\mathrm{I}\right.$, state $\left.{ }_{R A M}^{\prime}\right) \leftarrow \Pi$ ( state $_{R A M}$, inp);

6. If $\mathbf{I}=$ (stop, $z)$, then set state $\leftarrow\left((\right.$ start, $\left.\star)\left\|0^{\kappa}\right\| 0^{\log n}\right)$ and output (I, state);

7. Else set state $\leftarrow\left(\right.$ state $\left._{R A M}^{\prime}\left\|k_{\text {last }}\right\| \mathrm{I}\right)$ and output (I, state).

Theorem 6. Assume that $\mathcal{S K E}$ is an authenticated encryption scheme with ciphertexts size bounded by $q(\kappa, n) \in \operatorname{poly}(\kappa)$, and that $\mathcal{C S}$ is a $(3 q+n+\alpha+O(\log \kappa))$-leakage-resilient and continuously non-malleable code with split-state refresh, that additionally has $\alpha$-correlated codewords. Then, assuming the system can switch to safe mode for poly $(\kappa)$ times and self-destruct afterwards, the above construction defines a tamper-resilient $R A M$ compiler w.r.t. $\left(\mathcal{F}_{\text {bus }}, \mathcal{F}_{\text {mem }}\right)$, 
where $\mathcal{F}_{\text {bus }}$ and $\mathcal{F}_{\text {mem }}$ are, respectively, the class of split-state tampering functions applied on the bus and on the memory.

Proof. The proof proceeds similarly to the proof of Theorem 5. Let $L_{\mathrm{cptx}}^{i, j, f}$ be the leakage function that upon input $K_{j}$ first computes $\left.\tilde{\mathcal{E}} \leftarrow f\right|_{\text {mem }} ^{j}\left(K_{j}, \mathcal{E}\right)$, and then outputs $\tilde{\mathcal{E}}[i]$. Let $\left(\mathrm{S}_{0}, \mathrm{~S}_{1}\right)$ denote the simulator for $\mathcal{C S}$.

Simulator S:

1. Generate $\omega \leftarrow \mathrm{S}_{0}\left(1^{\kappa}\right)$, let $k \leftarrow \mathrm{KGen}\left(1^{\kappa}\right)$ a key for the authenticated encryption, create an encrypted dummy database $\mathcal{E}_{0}, \mathcal{E}_{1}$, where each entry is an encryption of $0^{\kappa}$, i.e. $\mathcal{E}_{0}, \mathcal{E}_{1}=$ $\left(c_{1}, \ldots, c_{n}\right)$ where $c_{i}=\operatorname{Enc}\left(k, 0^{\kappa} \| i\right)$. Run the simulator $\mathrm{S}_{1}$. Run the real world adversary A with input $\omega$. Let $f \lg _{\text {key }}$ be the flag that indicates the current state of the key. Initially the flag is set to OK. Let $f \lg _{\text {start }}$ be the flag that indicates the current state of the execution. Initially the flag is set to False. Set $h$ to be the identity function.

2. Upon receiving a query from the adversary A do the following:

If the query is for $\mathcal{O}_{\text {tamp }}$ and it is of the kind (TampMem, $f$ ), and $f \in \mathcal{F}_{\text {mem }}$, then set $h \leftarrow h \circ \overline{\circ f(\text { where }(h \circ f)}(x)=f(h(x)))$. Call $h$ the history tampering function.

If the query is for $\mathcal{O}_{\text {tamp }}$ and it is of the kind (TampBus, $f$ ), and $f \in \mathcal{F}_{\text {bus }}$, then set $g \leftarrow$ $(h \circ f)$. Call $g$ the current tampering function.

If the query is for doNext with input $x$, in case $f \lg _{\text {start }}=$ False then let $g$ be the current tampering function as defined above, and send the query (Tamp, $\left.\left.g\right|_{\text {key }}\left(\left(\cdot, \mathcal{E}_{0}\right),\left(\cdot, \mathcal{E}_{1}\right)\right)\right)$. (Namely, the restriction of $g$ to the encoding with the second part of the first (resp. second) input set to $\mathcal{E}_{0}\left(\operatorname{resp} . \mathcal{E}_{1}\right)$.) Do the following depending on the output of the simulator:

(a) If the simulator outputs $\diamond$, we set $f \lg _{\text {key }} \leftarrow$ OK.

(b) If the simulator outputs $\perp$, we set $f \lg _{\text {key }} \leftarrow$ broken.

(c) The last option for the simulator is to output a valid, but unrelated, key $k^{*}$. In this case, we set $f \lg _{\text {key }} \leftarrow$ unrelated.

Finally, set the flag $f \lg _{\text {start }}$ to True, and proceed as follows depending on $f l g_{\text {key }}$ :

(a') If $f l g_{\text {key }}=$ broken, then send the query $x$ to $\operatorname{Add}(\cdot)$ and answer with $\perp$.

(b') If $f \lg _{\text {key }}=$ unrelated then if $\mathrm{cnt}=0$ send the query $x$ to $\operatorname{Add}(\cdot)$; compute locally:

- If state $R A M=($ start, $\star)$ set inp $\leftarrow x$ and execute $\left(\right.$ I, state $\left.{ }_{R A M}^{\prime}\right) \leftarrow \Pi$ (state $R A M$, inp $)$, set state $\leftarrow\left(\right.$ state $\left._{R A M}^{\prime}\left\|k^{*}\right\| \mathrm{I}\right)$, increment cnt and return I;

- If $\mathbf{I}_{\text {last }}=($ read, $v)$ send the leakage oracle queries (Leak, $\left.\left(0, L_{\mathrm{cptx}}^{v, 0}\right)\right)$ and (Leak, $(1$, $\left.L_{\text {cptx }}^{v, 1}\right)$ ) to the simulator $\mathrm{S}_{1}$. Let $\widetilde{c}_{v}$ and $\widetilde{c}_{v}$ be the respective answers. Decrypt $\left(d^{\prime} \| i^{\prime}\right) \leftarrow \operatorname{Dec}\left(k^{*}, \widetilde{c}_{v}\right)$. If $\widetilde{c}_{v} \neq \widetilde{c}_{v}^{\prime}$ or $\left(d^{\prime} \| i^{\prime}\right)=\perp$ or $i^{\prime} \neq v$ then output $\perp$. Otherwise, set inp $\leftarrow d^{\prime}$ and compute locally (I, state $\left.{ }_{R A M}^{\prime}\right) \leftarrow \Pi$ (state ${ }_{R A M}$, inp) set state $\leftarrow\left(\operatorname{state}_{R A M}^{\prime}\left\|k^{*}\right\| \mathbf{I}\right)$ increment cnt and return I.

- If $\mathbf{I}_{\text {last }}=($ stop, $v)$ output $\mathcal{I}$, set state $\leftarrow\left((\right.$ start, $\left.\star),\left\|0^{\kappa}\right\| 0^{\log n}\right)$ and reset cnt to 0 and return $\mathrm{I}$.

(c') If $f \lg _{\text {key }}=\mathrm{OK}$ then, if $\mathrm{cnt}=0$, forward the query $x$ to $\operatorname{Execute}(\Lambda, \cdot)$ and let $\mathcal{I}$ be the answer. Else, if cnt $>0$, retrieve $\mathcal{I}$. Parse $\mathcal{I}=\left(I_{1}, \ldots, I_{p}\right)$ for $p \in \mathbb{N}$. If $I_{\text {cnt }}$ is of the form (read, $v$ ) then send the leakage oracle queries (Leak, $\left(0, L_{\mathrm{cptx}}^{v, 0, g}\right)$ ) and (Leak, $\left.\left(1, L_{\mathrm{cptx}}^{v, 1, g}\right)\right)$ to the simulator $\mathrm{S}_{1}$. Let $\widetilde{c}_{v}$ and $\widetilde{c}_{v}$ be the respective answers. If $\widetilde{c}_{v}=\mathcal{E}[v]=\widetilde{c}_{v}^{\prime}$ then output $\mathrm{I}_{\mathrm{cnt}}$ and increment the counter, else output $\perp$. 
In all of the above cases, whenever the value $\perp$ is returned to the adversary reset the flag $f \mathrm{lg}_{\text {key }}$ to OK and the flag $\mathrm{fl} \mathrm{lg}_{\text {start }}$ to False and set cnt to 0 and run the following simulated consistency check:

(a) Let $h$ be the history tampering function, and set $\zeta=q(\kappa, n) \cdot \omega(\log \kappa)$. Send the query $\left(\right.$ Final, $\left.h\right|_{\text {key }}\left(\left(\cdot, \mathcal{E}_{0}\right),\left(\cdot, \mathcal{E}_{1}\right)\right)$ to the simulator.

(b) If the simulator outputs $\perp$, then self-destruct.

(c) Else (namely, unrelated and same) Sample $i_{1}, \ldots, i_{\zeta} \leftarrow[n]$ and for $l \in[\zeta]$ send the leakage query (Leak, $\left.\left(0, L_{\text {cptx }}^{i_{l}, 0, h}\right)\right)$ and (Leak, $\left.\left(1, L_{\text {cptx }}^{i_{l}, 1, h}\right)\right)$ check if the returned values are different then self-destruct else continue.

(d) Leak the full containment of the encrypted databases. Specifically for $i \in[n]$ send the leakage query (Leak, $\left.\left(0, L_{\text {cptx }}^{i_{l}, 0, h}\right)\right)$ and $\left(\right.$ Leak, $\left.\left(1, L_{\text {cptx }}^{i_{l}, 1, h}\right)\right)$. Let $\tilde{c}_{i}^{0}$ and $\tilde{c}_{i}^{1}$ be the answers. Set $\mathcal{E}_{0} \leftarrow\left(c^{0}{ }_{1}, \ldots, c^{0}{ }_{n}\right)$ and $\mathcal{E}_{1} \leftarrow\left(c^{1}{ }_{1}, \ldots, c^{1}{ }_{n}\right)$.

(e) If the simulator of $\mathcal{C S}$ outputs $\tilde{K}_{0}, \tilde{K}_{1}$ then the simulation can continue by executing the code of TamperExec on the database $\left(\tilde{K}_{0}, \mathcal{E}_{0}\right),\left(\tilde{K}_{1}, \mathcal{E}_{1}\right)$.

We prove the indistinguishability in 3 steps. Let $\mathrm{H}_{i}$ be the $i$-th hybrid adversary, such adversary takes as input $1^{\kappa}$ and the original database $\mathcal{D}$ and interact with doNext. The latter defines the hybrid experiment $\mathbf{H}_{i}$, specifically $\mathbf{H}_{i}(\kappa):=\mathrm{D}\left(\mathrm{H}_{i}\left(1^{\kappa}, \mathcal{D}\right) \leftrightarrows \operatorname{Execute}(\Lambda, \cdot), \operatorname{Add}(\cdot)\right)$ where $\Lambda=(\Pi, \mathcal{D})$. Let the hybrid adversaries be defined as follow:

Hybrid Adversary $\mathrm{H}_{1}$. It takes as input the database $\mathcal{D}$ and executes the same code of the simulator $\mathrm{S}$, but it honestly encrypts the database $\mathcal{D}$. Namely, $\mathcal{E}=\left(c_{1}, \ldots, c_{n}\right)$ where $c_{i} \leftarrow \operatorname{Enc}\left(k,\left(d_{i} \| i\right)\right)$ for all $1 \leq i \leq n$.

Hybrid Adversary $\mathrm{H}_{2}$. It takes as input the database $\mathcal{D}$ and executes the same code of the hybrid adversary $\mathrm{H}_{1}$, but in point 2.(c') it does not check that $\widetilde{c}_{v}=\mathcal{E}[v]=\widetilde{c}_{v}$ (where $\widetilde{c}_{v}:=L_{\mathrm{cptx}}^{v, 0, g}\left(K_{0}\right)$ and $\left.\widetilde{c}_{v}:=L_{\mathrm{cptx}}^{v, 1, g}\left(K_{1}\right)\right)$. Instead it decrypts $\left(d^{\prime} \| i^{\prime}\right)=\operatorname{Dec}\left(k, \widetilde{c}_{v}\right)$, and if $\widetilde{c}_{v} \neq \widetilde{c}_{v}$ or $\left(d^{\prime} \| i^{\prime}\right)=\perp$ or $i^{\prime} \neq v$ then outputs $\perp$ and refreshes $K_{0}, K_{1}$. (Namely, it executes the same code the compiler CompNext would execute.)

Hybrid Adversary $\mathrm{H}_{3}$. It takes as input the database $\mathcal{D}$ and executes the same code of the hybrid adversary $\mathrm{H}_{2}$, but instead of running the simulator $\mathrm{S}_{1}$ of the R-CNMC, it samples $\left(K_{0}, K_{1}\right) \leftarrow \operatorname{Encode}(\omega, k)$, computes the decoding, the leakage function $L_{\text {cptx }}$ and the refreshing algorithm directly on $K_{0}, K_{1}$. In particular, whenever it computes a decoding it does the following checks. If the decoded key $k^{*}$ is $\perp$ then set the flag $f \lg _{\text {key }} \leftarrow$ broken, else if $k^{*}$ is equal to the original key $k$ then set $f l g_{\text {key }} \leftarrow \mathrm{OK}$, else set $f l g_{\text {key }} \leftarrow$ unrelated.

Lemma 25. For any read-only $R A M \Lambda=(\Pi, \mathcal{D})$ there exists a negligible function $\nu: \mathbb{N} \rightarrow[0,1]$ such that $\mid \mathbb{P}\left[\mathbf{H}_{1}(\kappa)=1\right]-\mathbb{P}\left[\right.$ IdealExec $\left._{S, \Lambda}(\kappa)=1\right] \mid \leq \nu(\kappa)$.

The proof of the lemma is identical to that of Lemma 21, and is therefore the proof is omitted.

Lemma 26. For any read-only $R A M \Lambda=(\Pi, \mathcal{D})$ there exists a negligible function $\nu: \mathbb{N} \rightarrow[0,1]$ such that $\left|\mathbb{P}\left[\mathbf{H}_{1}(\kappa)=1\right]-\mathbb{P}\left[\mathbf{H}_{2}(\kappa)=1\right]\right| \leq \nu(\kappa)$.

The proof of the lemma is identical to that of Lemma 22, and is therefore omitted.

Lemma 27. For any read-only $R A M \Lambda=(\Pi, \mathcal{D})$ there exists a negligible function $\nu: \mathbb{N} \rightarrow[0,1]$ such that $\left|\mathbb{P}\left[\mathbf{H}_{2}(\kappa)=1\right]-\mathbb{P}\left[\mathbf{H}_{3}(\kappa)=1\right]\right| \leq \nu(\kappa)$. 
Proof sketch. Here, we use the security of the R-CNMC. There are two things to notice. First, the function $\left.g\right|_{\text {key }}$ for any assignment of the encrypted databases $\mathcal{E}_{0}, \mathcal{E}_{1}$ is a valid split-state tampering function. Second, by continuously tampering with the encrypted split-state database, for each round, the adversary can leak information on $K_{0}, K_{1}$. In particular, both hybrid adversaries computes a sequence of leakage functions on $K_{0}, K_{1}$ from the set $\mathcal{L}$ defined $^{16}$ below:

$$
\mathcal{L}:=\left\{\left(L_{\mathrm{cptx}}^{i, 0, f}, L_{\mathrm{cptx}}^{i, 1, f}\right): i \in \mathbb{N}, f \in\{g, h\}\right\} .
$$

We show that, for every round, the sequence of leakage functions applied during that round is below the leakage bound of $\mathcal{C S}$. Fix a round, let $\bar{L}:=\left(\left(L_{1}^{0}, L_{1}^{1}\right),\left(L_{2}^{0}, L_{2}^{1}\right), \ldots,\left(L_{p}^{0}, L_{p}^{1}\right)\right)$ be the sequence of leakage functions sent by the simulator before $\perp$ is triggered $\left(p=\kappa^{c}\right.$ is a polynomial). Let $L_{\text {check }}\left(K_{0}, K_{1}\right)$ be the list of leakage functions that the simulator does during the simulated consistency check. Specifically:

$\underline{L_{\text {check }}\left(K_{0}, K_{1}\right)}:$

- Samples $i_{1}, \ldots, i_{\zeta} \leftarrow[n]$

- For $l \in[\zeta]$ and $j \in\{0,1\}$ it computes $C_{l}^{j}:=L_{\text {cptx }}^{i_{l}, 0, h}\left(K_{k}\right)$, if $C_{l}^{0} \neq C_{l}^{1}$ the it stops the cycle and outputs the list $\left\{\left(C_{1}^{0}, C_{1}^{1}\right), \ldots,\left(C_{l}^{0}, C_{l}^{1}\right)\right\}$.

- Outputs the list $\left\{\left(L_{\text {cptx }}^{i, 0, h}\left(K_{0}\right), L_{\text {cptx }}^{i, 0, h}\left(K_{0}\right)\right): i \in[n]\right\}$.

We bound the average conditional min-entropy of $K_{j}$, where $j \in\{0,1\}$, given the view of the simulator.

$$
\begin{aligned}
& \widetilde{\mathbb{H}}_{\infty}\left(K_{j} \mid \bar{L}\left(K_{0}, K_{1}\right), L_{\text {check }}\left(K_{0}, K_{1}\right)\right) \\
& \quad \geq \widetilde{\mathbb{H}}_{\infty}\left(K_{j} \mid L_{1}^{j}\left(K_{j}\right), \ldots, L_{p}^{j}\left(K_{j}\right), K_{1-j}, L_{\text {check }}\left(K_{0}, K_{1}\right)\right) \\
& \quad \geq \widetilde{\mathbb{H}}_{\infty}\left(K_{j} \mid L_{1}^{1-j}\left(K_{1-j}\right), \ldots, L_{p-1}^{1-j}\left(K_{1-j}\right), L_{p}^{j}\left(K_{j}\right), K_{1-j}, L_{\text {check }}\left(K_{0}, K_{1}\right)\right) \\
& \quad \geq \widetilde{\mathbb{H}}_{\infty}\left(K_{j} \mid L_{1}^{1-j}\left(K_{1-j}\right), \ldots, L_{p-1}^{1-j}\left(K_{1-j}\right), L_{p}^{j}\left(K_{j}\right), K_{1-j}, L_{\text {check }}\left(K_{0}, K_{1}\right)\right) \\
& \quad \geq \widetilde{\mathbb{H}}_{\infty}\left(K_{j} \mid p, L_{p}^{j}\left(K_{j}\right), K_{1-j}, L_{\text {check }}\left(K_{0}, K_{1}\right)\right) \\
& \geq \widetilde{\mathbb{H}}_{\infty}\left(K_{j} \mid K_{1-j}, L_{\text {check }}\left(K_{0}, K_{1}\right)\right)-O(\log \kappa)-q(\kappa, n) .
\end{aligned}
$$

In the above computation, the first equation follows by the fact that, for any $i$, the value $L_{i}^{1-j}\left(K_{1-j}\right)$ is a function of $K_{1-j}$, the second equation follows by the fact that for any $i<p$, by definition of $\mathrm{H}_{1}$ and $\mathrm{H}_{2}, L_{i}^{1-j}\left(K_{1-j}\right)=L_{i}^{j}\left(K_{j}\right)$ holds, and in the forth equation we applied the bound on the size of a ciphertext for $\mathcal{S} \mathcal{K} \mathcal{E}$.

It is left to give a bound on $\widetilde{\mathbb{H}}_{\infty}\left(K_{j} \mid K_{1-j}, L_{\text {check }}\left(K_{0}, K_{1}\right)\right)$. Let $\left(L_{1}^{\prime 0}, L_{1}^{\prime 1}\right), \ldots,\left(L_{p^{\prime}}^{\prime 0}, L_{p^{\prime}}^{\prime 1}\right)$ where $p^{\prime} \leq n$ be the output of $L_{\text {check. }}$. Let $X$ be a random variable that counts the number of indexes $i^{\prime}$ such that $L_{i^{\prime}}^{\prime 0} \neq L_{i^{\prime}}^{\prime 1}$. By following the same reasoning as before, it can be shown that:

$$
\widetilde{\mathbb{H}}_{\infty}\left(K_{j} \mid K_{1-j}, L_{\text {check }}\left(K_{0}, K_{1}\right)\right) \geq \widetilde{\mathbb{H}}_{\infty}\left(K_{j} \mid K_{1-j}\right)-\mathbb{E}[X] q(k+\log n) .
$$

Let Abort be the indicator random variable for the check $C_{l}^{0} \neq C_{l}^{1}$ in the computation of $L_{\text {check. }}$. Let $B:=n / q(\kappa, n)$, since $X \in \mathbb{N}$ we have that

$$
\begin{aligned}
\mathbb{E}[X] & =\sum_{i} i \mathbb{P}[X=i] \\
& =\sum_{i} i \mathbb{P}[X=i \wedge \text { Abort }=0]+\sum_{i} i \mathbb{P}[X=i \wedge \text { Abort }=1] \\
& \leq(n \mathbb{P}[X>B \wedge \text { Abort }=0]+B)+1,
\end{aligned}
$$

\footnotetext{
${ }^{16}$ Notice that the leakage functions depend $s$ on $\left.g\right|_{\text {mem }}$ which can change during the execution of one round. For simplicity we hide this extra parameter.
} 
where in the last equation we used that when Abort $=1$ then $X=1$, and that $X \leq n$. We show that the probability that $X>B$ and Abort $=0$ is negligible. Let $Z$ be equal to the sum of $\zeta$ independent identically distributed Bernoulli random variables with parameter $B / n$. It is easy to see that

$$
\mathbb{P}[X>B \wedge \text { Abort }=0] \leq \mathbb{P}[\text { Abort }=0 \mid X>B] \leq \mathbb{P}[Z=0]=(1-B / n)^{\zeta} .
$$

The latter, by our choice of the parameter $\zeta=q(\kappa, n) \cdot \omega(\log \kappa)$, is negligible. Therefore we have that $\mathbb{E}[X] \leq n / q(\kappa, n)+2$. Summing up together the amount of leakage performed by the simulator, we get $O(\log \kappa)+3 q(\kappa, n)+n+\alpha$ as required in the statement of the theorem.

Lemma 28. $\mathbb{P}\left[\mathbf{H}_{3}(\kappa)=1\right]=\mathbb{P}\left[\mathrm{D}\left(\operatorname{TamperExec}_{\mathrm{A}, \Sigma, \Lambda}^{\mathcal{F}_{\text {bus }}, \mathcal{F}_{\text {mem }}}(\kappa)\right)=1\right]$.

Proof sketch. For each query to doNext with input $x$, the hybrid experiment checks the flag flg $g_{\text {key }}$. In particular:

- If the flag is set to broken then it means that $\operatorname{Decode}\left(\widetilde{K}_{0}, \widetilde{K}_{1}\right)$ is $\perp$ (by inspection), therefore in this case the real experiment would run, add $x$ to the set of queries and output $\perp$; the hybrid $\mathbf{H}_{3}$ does the same using its interface Add.

- If the flag is set to unrelated then it means that $\operatorname{Decode}\left(\widetilde{K}_{0}, \widetilde{K}_{1}\right)=k^{*} \neq k$ (by inspection). In this case the hybrid experiment simulates perfectly the real experiment by computing locally as would happen in the real experiment, moreover the hybrid adds the query to $\mathcal{Q}$ using its interface Add.

- If the flag is set to OK then it means that Decode $\left(\widetilde{K}_{0}, \widetilde{K}_{1}\right)=k$. In this case the hybrid experiment uses its interface Execute, but before outputting the transcript it processes it by finding the point where the real experiment would output $\perp$ (if the latter, indeed, outputs $\perp$ ).

Further, notice that, for each round, in $\mathbf{H}_{3}$ the tampered database is computed by applying the function $\left.h\right|_{\text {mem }}$ where $h$ is a function of the current codeword and the current tampered database. Also, $h$ is the concatenation of all the persistent tampering functions in the current round. In particular, to build the tampered database for the next round, the hybrid experiment computes it exactly as mentioned above. Therefore, by induction on the number of rounds, the tampered database, as a random variable, is distributed exactly as in the real experiment.

\section{Conclusion}

We have studied a generalization of the concept of continuously non-malleable codes, for the split-state model, where after a decoding error happens the original codeword gets refreshed instead of triggering a self-destruct. Importantly, the refreshing happens locally on each share of the codeword, without requiring any communication between the two parts. As we have shown, this extra feature allows to avoid self-destruct in some of the applications of non-malleable codes. It also allows naturally to obtain security against continual leakage attacks.

Open problems include whether stronger forms of non-malleability in the split-state model can be achieved, while at the same time allowing for split-state refreshing, and whether refreshable continuously non-malleable codes in the split-state model can be achieved without relying on setup assumptions. 


\section{References}

[1] Divesh Aggarwal, Shashank Agrawal, Divya Gupta, Hemanta K. Maji, Omkant Pandey, and Manoj Prabhakaran. Optimal computational split-state non-malleable codes. In $T C C$, pages 393-417, 2016.

[2] Divesh Aggarwal, Yevgeniy Dodis, Tomasz Kazana, and Maciej Obremski. Non-malleable reductions and applications. In STOC, pages 459-468, 2015.

[3] Divesh Aggarwal, Yevgeniy Dodis, and Shachar Lovett. Non-malleable codes from additive combinatorics. In STOC, pages 774-783, 2014.

[4] Divesh Aggarwal, Stefan Dziembowski, Tomasz Kazana, and Maciej Obremski. Leakageresilient non-malleable codes. In TCC, pages 398-426, 2015.

[5] Divesh Aggarwal, Tomasz Kazana, and Maciej Obremski. Inception makes non-malleable codes stronger. In TCC, pages 319-343, 2017.

[6] Shashank Agrawal, Divya Gupta, Hemanta K. Maji, Omkant Pandey, and Manoj Prabhakaran. Explicit non-malleable codes against bit-wise tampering and permutations. In CRYPTO, pages 538-557, 2015.

[7] Shashank Agrawal, Divya Gupta, Hemanta K. Maji, Omkant Pandey, and Manoj Prabhakaran. A rate-optimizing compiler for non-malleable codes against bit-wise tampering and permutations. In TCC, pages 375-397, 2015.

[8] Marshall Ball, Dana Dachman-Soled, Mukul Kulkarni, and Tal Malkin. Non-malleable codes for bounded depth, bounded fan-in circuits. In EUROCRYPT, pages 881-908, 2016.

[9] Marshall Ball, Dana Dachman-Soled, Mukul Kulkarni, and Tal Malkin. Non-malleable codes from average-case hardness: $\mathrm{AC} 0$, decision trees, and streaming space-bounded tampering. In EUROCRYPT, pages 618-650, 2018.

[10] Eli Biham and Adi Shamir. Differential fault analysis of secret key cryptosystems. In CRYPTO, pages 513-525, 1997.

[11] Dan Boneh, Xavier Boyen, and Hovav Shacham. Short group signatures. In CRYPTO, pages $41-55,2004$.

[12] Dan Boneh, Richard A. DeMillo, and Richard J. Lipton. On the importance of checking cryptographic protocols for faults (extended abstract). In EUROCRYPT, pages 37-51, 1997.

[13] Nishanth Chandran, Vipul Goyal, Pratyay Mukherjee, Omkant Pandey, and Jalaj Upadhyay. Block-wise non-malleable codes. In ICALP, pages 31:1-31:14, 2016.

[14] Nishanth Chandran, Bhavana Kanukurthi, and Srinivasan Raghuraman. Informationtheoretic local non-malleable codes and their applications. In TCC, pages 367-392, 2016.

[15] Melissa Chase, Markulf Kohlweiss, Anna Lysyanskaya, and Sarah Meiklejohn. Malleable proof systems and applications. In EUROCRYPT, pages 281-300, 2012.

[16] Eshan Chattopadhyay and Xin Li. Non-malleable codes and extractors for small-depth circuits, and affine functions. In STOC, pages 1171-1184, 2017. 
[17] Eshan Chattopadhyay and Xin Li. Non-malleable extractors and codes in the interleaved split-state model and more. Electronic Colloquium on Computational Complexity, TR18070, 2018. https://eccc.weizmann.ac.il/report/2018/070/.

[18] Eshan Chattopadhyay and David Zuckerman. Non-malleable codes against constant splitstate tampering. In FOCS, pages 306-315, 2014.

[19] Mahdi Cheraghchi and Venkatesan Guruswami. Capacity of non-malleable codes. In Innovations in Theoretical Computer Science, pages 155-168, 2014.

[20] Mahdi Cheraghchi and Venkatesan Guruswami. Non-malleable coding against bit-wise and split-state tampering. In TCC, pages 440-464, 2014.

[21] Sandro Coretti, Yevgeniy Dodis, Björn Tackmann, and Daniele Venturi. Non-malleable encryption: Simpler, shorter, stronger. In TCC, volume 2015, pages 306-335, 2016.

[22] Sandro Coretti, Ueli Maurer, Björn Tackmann, and Daniele Venturi. From single-bit to multi-bit public-key encryption via non-malleable codes. In TCC, pages 532-560, 2015.

[23] Dana Dachman-Soled and Mukul Kulkarni. Upper and lower bounds for continuous nonmalleable codes. Cryptology ePrint Archive, Report 2018/517, 2018. https://eprint. iacr.org/2018/517.

[24] Dana Dachman-Soled, Feng-Hao Liu, Elaine Shi, and Hong-Sheng Zhou. Locally decodable and updatable non-malleable codes and their applications. In TCC, pages 427-450, 2015.

[25] Ivan Damgård, Sebastian Faust, Pratyay Mukherjee, and Daniele Venturi. Bounded tamper resilience: How to go beyond the algebraic barrier. In ASIACRYPT, pages 140-160, 2013.

[26] Ivan Damgård, Sebastian Faust, Pratyay Mukherjee, and Daniele Venturi. Bounded tamper resilience: How to go beyond the algebraic barrier. J. Cryptology, 30(1):152-190, 2017.

[27] Francesco Davì, Stefan Dziembowski, and Daniele Venturi. Leakage-resilient storage. In $S C N$, pages 121-137, 2010.

[28] Yevgeniy Dodis, Kristiyan Haralambiev, Adriana López-Alt, and Daniel Wichs. Cryptography against continuous memory attacks. In FOCS, pages 511-520, 2010.

[29] Yevgeniy Dodis, Allison B. Lewko, Brent Waters, and Daniel Wichs. Storing secrets on continually leaky devices. In FOCS, pages 688-697, 2011.

[30] Yevgeniy Dodis, Rafail Ostrovsky, Leonid Reyzin, and Adam Smith. Fuzzy extractors: How to generate strong keys from biometrics and other noisy data. SIAM J. Comput., 38(1):97-139, 2008.

[31] Stefan Dziembowski, Tomasz Kazana, and Maciej Obremski. Non-malleable codes from two-source extractors. In CRYPTO, pages 239-257, 2013.

[32] Stefan Dziembowski, Krzysztof Pietrzak, and Daniel Wichs. Non-malleable codes. In Innovations in Computer Science, pages 434-452, 2010.

[33] Antonio Faonio and Jesper Buus Nielsen. Non-malleable codes with split-state refresh. In PKC, pages 279-309, 2017.

[34] Antonio Faonio and Daniele Venturi. Efficient public-key cryptography with bounded leakage and tamper resilience. In ASIACRYPT, pages 877-907, 2016. 
[35] Sebastian Faust, Kristina Hostáková, Pratyay Mukherjee, and Daniele Venturi. Nonmalleable codes for space-bounded tampering. In CRYPTO, pages 95-126, 2017.

[36] Sebastian Faust, Pratyay Mukherjee, Jesper Buus Nielsen, and Daniele Venturi. Continuous non-malleable codes. In TCC, pages 465-488, 2014.

[37] Sebastian Faust, Pratyay Mukherjee, Jesper Buus Nielsen, and Daniele Venturi. A tamper and leakage resilient von Neumann architecture. In PKC, pages 579-603, 2015.

[38] Sebastian Faust, Pratyay Mukherjee, Daniele Venturi, and Daniel Wichs. Efficient nonmalleable codes and key-derivation for poly-size tampering circuits. In EUROCRYPT, pages 111-128, 2014.

[39] Sebastian Faust, Pratyay Mukherjee, Daniele Venturi, and Daniel Wichs. Efficient nonmalleable codes and key derivation for poly-size tampering circuits. IEEE Trans. Information Theory, 62(12):7179-7194, 2016.

[40] Eiichiro Fujisaki and Keita Xagawa. Public-key cryptosystems resilient to continuous tampering and leakage of arbitrary functions. In ASIACRYPT, pages 908-938, 2016.

[41] Rosario Gennaro, Anna Lysyanskaya, Tal Malkin, Silvio Micali, and Tal Rabin. Algorithmic tamper-proof (ATP) security: Theoretical foundations for security against hardware tampering. In TCC, pages 258-277, 2004.

[42] Sudhakar Govindavajhala and Andrew W. Appel. Using memory errors to attack a virtual machine. In IEEE Symposium on Security and Privacy, pages 154-165, 2003.

[43] Jens Groth and Amit Sahai. Efficient non-interactive proof systems for bilinear groups. In EUROCRYPT, pages 415-432, 2008.

[44] Shai Halevi and Huijia Lin. After-the-fact leakage in public-key encryption. In $T C C$, pages 107-124, 2011.

[45] Yael Tauman Kalai, Bhavana Kanukurthi, and Amit Sahai. Cryptography with tamperable and leaky memory. In CRYPTO, pages 373-390, 2011.

[46] Aggelos Kiayias, Feng-Hao Liu, and Yiannis Tselekounis. Practical non-malleable codes from l-more extractable hash functions. In CCS, pages 1317-1328, 2016.

[47] Aggelos Kiayias, Feng-Hao Liu, and Yiannis Tselekounis. Non-malleable codes for partial functions with manipulation detection. Cryptology ePrint Archive, Report 2018/538, 2018. https://eprint.iacr.org/2018/538.

[48] Xin Li. Improved non-malleable extractors, non-malleable codes and independent source extractors. In STOC, pages 1144-1156, 2017.

[49] Xin Li. Non-malleable extractors and non-malleable codes: Partially optimal constructions. Electronic Colloquium on Computational Complexity, TR18-028, 2018. https://eccc. weizmann.ac.il/report/2018/028/\#revision1.

[50] Feng-Hao Liu and Anna Lysyanskaya. Tamper and leakage resilience in the split-state model. In CRYPTO, pages 517-532, 2012.

[51] Moni Naor and Gil Segev. Public-key cryptosystems resilient to key leakage. In CRYPTO, volume 2009, pages 18-35, 2009. 
[52] Rafail Ostrovsky, Giuseppe Persiano, Daniele Venturi, and Ivan Visconti. Continuously non-malleable codes in the split-state model from minimal assumptions. Cryptology ePrint Archive, Report 2018/542, 2018. https://eprint.iacr.org/2018/542.

[53] Martin Otto. Fault Attacks and Countermeasures. PhD thesis, University of Paderborn, Germany, 2006. 